\title{
Mass and axial charge of heavy baryons
}

\author{
Nan Jiang Xiao-Lin Chen ${ }^{7}$ and Shi-Lin Zhu \\ ${ }^{1}$ Department of Physics and State Key Laboratory of Nuclear \\ Physics and Technology, Peking University, Beijing 100871, China \\ ${ }^{2}$ Collaborative Innovation Center of Quantum Matter, Beijing 100871, China
}

\begin{abstract}
We investigate the antitriplet and sextet heavy baryon systems with $J^{P}=\frac{1}{2}^{+}, \frac{3}{2}^{+}$in the framework of the heavy baryon chiral perturbation theory. We first calculate the chiral corrections to the heavy baryon mass from the $\mathrm{SU}(3)$ flavor breaking effect up to $O\left(p^{3}\right)$. Then we extend the same formalism to calculate the chiral corrections to the axial charges of the heavy baryons in the isospin symmetry limit.
\end{abstract}

PACS numbers: 12.39.Fe, 14.20.Lq, 11.40.Ha

\section{INTRODUCTION}

With the discovery of the Higgs Bosons, all particles within the standard model were established. However, the low energy behavior of the strong interaction remains extremely challenging due to the complicated infrared structure of quantum chromodynamics (QCD).

The discovery of the $J / \psi$ in 1974 [1, 2] opened the door to a new world of the heavy hadrons, which was accompanied by observation of the $\Upsilon$ family of mesons in $1977[3,4]$. Decades of research leads a wealth of experimental data on heavy baryons. The discovery of charm and bottom baryon states greatly enriches our knowledge of heavy quarks and heavy baryons. Meanwhile, the heavy baryons are not the simple copies of the known light baryons. The properties of the heavy flavor baryons and light flavor baryons are quite distinctive because the heavy baryon mass is much larger than the QCD scale $\Lambda_{\mathrm{QCD}} \approx 400 \mathrm{MeV}$.

Compared with the light mesons and baryons, the heavy flavored hadron systems containing a single heavy quark are particularly interesting. The structure of a heavy quark meson is very similar to a hydrogen atom in QED, which contains a heavy particle and a light one. A heavy meson can be regarded as a hydrogen atom of QCD. Likewise, a heavy baryon is similar to a helium atom. In this sense, the research of heavy baryons will provide us a more accurate test for QCD. In fact, there exists the additional heavy quark spin and flavor symmetry when the heavy quark mass goes to infinity. The observables can be expanded in terms of $1 / m_{Q}$ where $m_{Q}$ is the heavy quark mass. In addition, another motivation of the present work is that the nonanalytic corrections derived from the loop diagrams might reduce the error of extraction in the lattice calculation. We hope our results will be useful in the chiral extrapolation of the lattice simulation data.

In this work we focus on the heavy baryons containing a single heavy quark with either $\mathrm{C}=1$ or $\mathrm{B}=1$. The ground states satisfy the SU(4) symmetry in flavor space and form multiplets 20 (for spin- $\frac{1}{2}$ baryons) or 20 '(for spin- $\frac{3}{2}$ baryons). The spin- $\frac{1}{2}$ baryons include the octet $(C=0)$, antitriplet $(C=1)$, sextet $(C=1)$, and triplet $(C=2)$. The spin- $\frac{3}{2}$ baryons include the decuplet $(\mathrm{C}=0)$, sextet $(\mathrm{C}=1)$, triplet $(\mathrm{C}=2)$ and a singlet $(\mathrm{C}=3)$. The investigations of the mass, lifetime, and axial charge of the heavy baryons will help us understand the underlying structure of heavy baryons.

When the two light quarks within the ground state heavy baryon are in the flavor antitriplet, the quantum number of the heavy baryon is $J^{P}=\frac{1}{2}^{+}$. When the two light quarks are in the symmetric flavor sextet, the quantum number of the heavy baryon can be either $J^{P}=\frac{1}{2}^{+}$or $\frac{3}{2}^{+}$. In recent years, many charmed and bottomed baryons were observed experimentally [5]. The spectroscopic properties of some charm baryons were explored by CDF Collaboration [6] [7]. On the other hand, the scattering lengths of heavy baryons with Goldstone bosons were calculated in Refs. [8, 9]. The possible deuteronlike hadronic molecular states composed of two heavy baryons were investigated in Ref. [10]. The pionic coupling constants of the heavy baryons played an important role in the above work.

In this work, we will investigate the heavy antitriplet and sextet systems. We will calculate the chiral corrections to the heavy baryon mass and axial charge from the $\mathrm{SU}(3)$ flavor breaking effects by employing HBChPT. We adopt

\footnotetext{
*Electronic address: Jiangn@pku.edu.cn

${ }^{\dagger}$ Electronic address: chenxl@pku.edu.cn

${ }^{\ddagger}$ Electronic address: zhusl@pku.edu.cn
} 
the heavy quark limit and discard all the recoil corrections. We include the corrections up to $O\left(p^{3}\right)$ from both the strong and electromagnetic interaction. There were many references on the chiral corrections to the axial currents of the nucleon octet 11 14]. We adopt the same approach to study the axial charges of the heavy baryons.

This paper is organized as follows: In Sec. II, we introduce the effective chiral Lagrangians at the leading order. In Sec. III, we calculate the chiral corrections to the masses of the antitriplet and sextet baryon systems. In Sec. IV, we calculate the chiral corrections to the axial charges in the isospin symmetry limit. The last section is a short summary.

\section{THE HBCHPT FORMALISM}

The approximate chiral symmetry and its spontaneous breaking play an important role in the low energy hadron interaction. Chiral perturbation theory (ChPT) [15] provides a systematic expansion of the physical observables in terms of small momentum $p$ and the mass of Goldstone bosons $m$. In fact, ChPT has been widely used to study the lowenergy hadron interaction.

In the early stage, ChPT was employed to study the purely mesonic system [16, 17]. Later it was extended to discuss the baryon-meson system [11, 18 20]. At the lowest order, the couplings between the baryon and pseudoscalar mesons $(\pi, \mathrm{K}, \eta)$ are solely governed by chiral dynamics. With the consistent power counting scheme, one can construct the effective Lagrangians of the meson baryon system and calculate physical quantities order by order [21, 22].

In order to deal with the heavy baryon system, the heavy baryon chiral perturbation theory (HBChPT) was developed 23 26], which provides a convenient framework to make a dual expansion in terms of both the small momentum and $\frac{1}{M}$, where $M$ is the heavy baryon mass. On the other hand, the infrared regularization scheme was introduced to preserve both the power counting and analyticity in the framework of the relativistic baryon ChPT [27]. In this work, we use HBChPT to investigate the heavy baryon systems.

For the heavy baryons multiplet (Qqq), only the two light quarks participate in the flavor transformation with the heavy quark Q acting as a spectator. The pseudoscalar meson fields and spin- $\frac{1}{2}$ baryon multiplets are defined as follows

$$
\begin{gathered}
\phi=\left(\begin{array}{ccc}
\pi^{0}+\frac{1}{\sqrt{3}} \eta & \sqrt{2} \pi^{+} & \sqrt{2} K^{+} \\
\sqrt{2} \pi^{-} & -\pi^{0}+\frac{1}{\sqrt{3}} \eta & \sqrt{2} K^{0} \\
\sqrt{2} K^{-} & \sqrt{2} \bar{K}^{0} & -\frac{2}{\sqrt{3}} \eta
\end{array}\right) \\
B_{\overline{3}}=\left(\begin{array}{ccc}
0 & \Lambda_{c}^{+} & \Xi_{c}^{+} \\
-\Lambda_{c}^{+} & 0 & \Xi_{c}^{0} \\
-\Xi_{c}^{+} & -\Xi_{c}^{0} & 0
\end{array}\right), \quad B_{6}=\left(\begin{array}{ccc}
\Sigma_{c}^{++} & \frac{1}{\sqrt{2}} \Sigma_{c}^{+} & \frac{1}{\sqrt{2}} \Xi_{c}^{\prime+} \\
\frac{1}{\sqrt{2}} \Sigma_{c}^{+} & \Sigma_{c}^{0} & \frac{1}{\sqrt{2}} \Xi_{c}^{\prime 0} \\
\frac{1}{\sqrt{2}} \Xi_{c}^{\prime+} & \frac{1}{\sqrt{2}} \Xi_{c}^{\prime 0} & \Omega_{c}^{0}
\end{array}\right)
\end{gathered}
$$

The spin- $\frac{3}{2}$ baryons $B_{6}^{* \mu}$ are the so-called Rarita-Schwinger vector-spinor fields [28], which are similar to $B_{6}$. We adopt the nonlinear realization of the chiral symmetry and its spontaneous breaking and introduce the following building blocks.

$$
\begin{aligned}
U(x) & =e^{\frac{i}{F_{0}} \phi(x)}, \quad u^{2}=U \\
\Gamma_{\mu} & =\frac{1}{2}\left(u^{\dagger} \partial_{\mu} u+u \partial_{\mu} u^{\dagger}\right) \\
u_{\mu} & =\frac{i}{2}\left(u^{\dagger} \partial_{\mu} u-u \partial_{\mu} u^{\dagger}\right) \\
D_{\mu} B & =\partial_{\mu} B+\Gamma_{\mu} B+B \Gamma_{\mu}^{T}
\end{aligned}
$$

The superscript $T$ denotes the transpose in the flavor space. The pion decay constant $F_{0} \approx 92.4 \mathrm{MeV}$. The leading order pseudoscalar meson and heavy baryon Lagrangian is $O\left(p^{1}\right)$, which reads [29]

$$
\begin{aligned}
\mathcal{L}_{0}^{(1)}= & \frac{1}{2} \operatorname{tr}\left[\bar{B}_{\overline{3}}\left(i \not D-M_{\overline{3}}\right) B_{\overline{3}}\right]+\operatorname{tr}\left[\bar{B}_{6}\left(i \not D-M_{6}\right) B_{6}\right] \\
& +\operatorname{tr}\left\{\bar{B}_{6}^{* \mu}\left[-g_{\mu \nu}\left(i \not D-M_{6}^{*}\right)+i\left(\gamma_{\mu} D_{\nu}+\gamma_{\nu} D_{\mu}\right)-\gamma_{\mu}\left(i \not D+M_{6}^{*}\right) \gamma_{\nu}\right] B_{6}^{* \nu}\right\} \\
\mathcal{L}_{\mathrm{int}}^{(1)}= & g_{1} \operatorname{tr}\left(\bar{B}_{6} \psi \gamma_{5} B_{6}\right)+g_{2}\left[\operatorname{tr}\left(\bar{B}_{6} \psi \gamma_{5} B_{\overline{3}}\right)+h . c .\right]+g_{3}\left[\operatorname{tr}\left(\bar{B}_{6}^{* \mu} u_{\mu} B_{6}\right)+\text { h.c. }\right] \\
& +g_{4}\left[\operatorname{tr}\left(\bar{B}_{6}^{* \mu} u_{\mu} B_{\overline{3}}\right)+\text { h.c. }\right]+g_{5} \operatorname{tr}\left(\bar{B}_{6}^{* \nu} \psi \gamma_{5} B_{6 \nu}^{*}\right)+g_{6} \operatorname{tr}\left(\bar{B}_{\overline{3}} \psi \gamma_{5} B_{\overline{3}}\right)
\end{aligned}
$$


where $\mathcal{L}_{0}^{(1)}$ is the free part and $\mathcal{L}_{\text {int }}^{(1)}$ contains the interaction at $O\left(p^{1}\right)$. From the quark model and flavor $\mathrm{SU}(3)$ symmetry, the axial coupling constants $g_{1}=0.98[8,9], g_{1}=-\sqrt{\frac{8}{3}} g_{2}$ [29]. The heavy quark spin flavor symmetry leads to the following relations among these coupling constants, i.e., $g_{3}=\frac{\sqrt{3}}{2} g_{1}, g_{5}=-\frac{3}{2} g_{1}, g_{4}=-\sqrt{3} g_{2}[29]$. Within the antitriplet, the total angular momentum of the two light quarks is zero. The conservation of the angular moment and parity forbids the coupling of pseudoscalar mesons with the antitriplet heavy baryons, hence $g_{6}=0$. However, we keep the $g_{6}$-related terms in formulas till the numerical analysis.

The heavy baryon formulation of ChPT consists in an expansion in terms of $\frac{k}{4 \pi F_{0}}$ and $\frac{k}{M_{B}}$, where $k$ is the small residual component of external baryon. In the framework of $\mathrm{HBChPT}$, the baryon field $B$ is decomposed into the large (or light) component $\mathcal{N}$ and the small (or heavy) component $\mathcal{H}$. By using the path integral theory, the small component can be integrated out. Thus, the reduced effective Lagrangian only relies on the large component [21]. Their relationships are

$$
\begin{aligned}
& B=e^{-i m v \cdot x}(\mathcal{N}+\mathcal{H}) \\
& \mathcal{N}=e^{i m v \cdot x} \frac{1+\psi}{2} B, \quad \mathcal{H}=e^{i m v \cdot x} \frac{1-\psi}{2} B,
\end{aligned}
$$

where $v^{\mu}$ is the on shell velocity. For the spin- $\frac{3}{2}$ baryon, the large component is denoted as $\mathcal{T}^{\mu}$. In the heavy quark limit, the nonrelativistic Lagrangian reads

$$
\begin{aligned}
\hat{\mathcal{L}}^{(1)}= & \frac{1}{2} \operatorname{tr}\left[\overline{\mathcal{N}}_{\overline{3}}(i v \cdot D) \mathcal{N}_{\overline{3}}\right]+\operatorname{tr}\left[\overline{\mathcal{N}}_{6}\left(i v \cdot D-\delta_{1}\right) \mathcal{N}_{6}\right]+\operatorname{tr}\left\{\overline{\mathcal{T}}^{\rho}\left[-g_{\rho \sigma}\left(i v \cdot D-\delta_{2}\right)\right] \mathcal{T}^{\sigma}\right\} \\
& +2 g_{1} \operatorname{tr}\left(\overline{\mathcal{N}}_{6} S \cdot u \mathcal{N}_{6}\right)+2 g_{2}\left[\operatorname{tr}\left(\overline{\mathcal{N}}_{6} S \cdot u \mathcal{N}_{\overline{3}}\right)+h . c .\right]+g_{3}\left[\operatorname{tr}\left(\overline{\mathcal{T}}^{\mu} u_{\mu} \mathcal{N}_{6}\right)+h . c .\right] \\
& +g_{4}\left[\operatorname{tr}\left(\overline{\mathcal{T}}^{\mu} u_{\mu} \mathcal{N}_{\overline{3}}\right)+h . c .\right]+2 g_{5} \operatorname{tr}\left(\overline{\mathcal{T}}^{\nu} S \cdot u \mathcal{T}_{\nu}\right)+2 g_{6} \operatorname{tr}\left(\overline{\mathcal{N}}_{\overline{3}} S \cdot u \mathcal{N}_{\overline{3}}\right)
\end{aligned}
$$

The mass difference parameters are defined as $\delta_{1}=M_{6}-M_{\overline{3}}, \delta_{2}=M_{6^{*}}-M_{6}$. In the isospin symmetry limit, $\delta_{1}=126.52 \mathrm{MeV}, \delta_{2}=67.03 \mathrm{MeV}$. Here, we choose the average mass of the spin- $\frac{1}{2}$ antitriplet, sextet and spin- $\frac{3}{2}$ sextet baryons as $M_{\overline{3}}=2286.46 \mathrm{MeV}, M_{6}=2454.02 \mathrm{MeV}, M_{6^{*}}=2518.4 \mathrm{MeV}$ respectively [5].

The $\mathrm{SU}(3)$ flavor symmetry-breaking (SB) Lagrangian at $O\left(p^{2}\right)$ reads

$$
\begin{aligned}
\mathcal{L}_{b c}^{(2)}= & b_{1} \operatorname{tr}\left(\bar{B}_{6} \chi_{+} B_{6}\right)+b_{5} g_{\mu \nu} \operatorname{tr}\left(\bar{B}_{6}^{* \mu} \chi_{+} B_{6}^{* \nu}\right)+b_{6} \operatorname{tr}\left(\bar{B}_{\overline{3}} \chi_{+} B_{\overline{3}}\right) \\
& +c_{1} \operatorname{tr}\left(\bar{B}_{6} B_{6}\right) \operatorname{tr} \chi_{+}+c_{5} g_{\mu \nu} \operatorname{tr}\left(\bar{B}_{6}^{* \mu} B_{6}^{* \nu}\right) \operatorname{tr} \chi_{+}+c_{6} \operatorname{tr}\left(\bar{B}_{\overline{3}} B_{\overline{3}}\right) \operatorname{tr} \chi_{+}
\end{aligned}
$$

where

$$
\begin{gathered}
\chi=2 B_{0} \mathcal{M}, \quad \mathcal{M}=\operatorname{diag}\left(m_{u}, m_{d}, m_{s}\right) \\
\chi_{+}=u^{\dagger} \chi u^{\dagger}+u \chi^{\dagger} u=2 \chi+O\left(\phi^{2}\right)
\end{gathered}
$$

$m_{u}, m_{d}, m_{s}$ are the $u, d, s$ quark mass. The constant $B_{0}$ is related to the quark condensate. The method of constructing the chiral effective Lagrangian can be found in Ref. [30].

The mass splitting not only arises from the up and down quark mass difference but also from the different heavy baryon electric charges within an isospin multiplet. The QED Lagrangian at $O\left(p^{2}\right)$ reads

$$
\begin{aligned}
\mathcal{L}_{\mathrm{QED}}^{(2) 66}= & e_{1}^{66} \operatorname{tr}\left(\bar{B}_{6} Q_{+}^{2} B_{6}\right)+e_{2}^{66} \operatorname{tr}\left(\bar{B}_{6} Q_{+} B_{6}\right) \operatorname{tr} Q_{+}+e_{3}^{66} \operatorname{tr}\left(\bar{B}_{6} B_{6}\right) \operatorname{tr} Q_{+}^{2} \\
& +e_{4}^{66} \operatorname{tr}\left(\bar{B}_{6} Q_{+} B_{6} Q_{+}^{T}\right) \\
\mathcal{L}_{\mathrm{QED}}^{(2) \overline{3} \overline{3}}= & e_{1}^{\overline{3} \overline{3}} \operatorname{tr}\left(\bar{B}_{\overline{3}} Q_{+}^{2} B_{\overline{3}}\right)+e_{2}^{\overline{3} \overline{3}} \operatorname{tr}\left(\bar{B}_{\overline{3}} Q_{+} B_{\overline{3}}\right) \operatorname{tr} Q_{+}+e_{3}^{\overline{3} \overline{3}} \operatorname{tr}\left(\bar{B}_{\overline{3}} B_{\overline{3}}\right) \operatorname{tr} Q_{+}^{2} \\
\mathcal{L}_{\mathrm{QED}}^{(2) 6^{*} 6^{*}}= & e_{1}^{6^{*} 6^{*}} g_{\rho \sigma} \operatorname{tr}\left(\bar{B}_{6}^{* \rho} Q_{+}^{2} B_{6}^{* \sigma}\right)+e_{2}^{6 *} 6^{*} g_{\rho \sigma} \operatorname{tr}\left(\bar{B}_{6}^{* \rho} Q_{+} B_{6}^{* \sigma}\right) \operatorname{tr} Q_{+}+e_{3}^{6^{*} 6^{*}} g_{\rho \sigma} \operatorname{tr}\left(\bar{B}_{6}^{* \rho} B_{6}^{* \sigma}\right) \operatorname{tr} Q_{+}^{2} \\
& +e_{4}^{6^{*} 6^{*}} g_{\rho \sigma} \operatorname{tr}\left(\bar{B}_{6}^{* \rho} Q_{+} B_{6}^{* \sigma} Q_{+}^{T}\right)
\end{aligned}
$$

where

$$
\begin{gathered}
Q_{+}=\frac{1}{2}\left(u^{\dagger} Q u+u Q u^{\dagger}\right) \\
Q=2 q_{l}+q_{c} I=e \operatorname{diag}(2,0,0)
\end{gathered}
$$




$$
q_{l}=e \operatorname{diag}\left(\frac{2}{3},-\frac{1}{3},-\frac{1}{3}\right), \quad q_{c}=\frac{2}{3} e
$$

To some extent, the above effective Lagrangians mimic the electromagnetic spin-flavor interaction in the quark model, which arises from the hard photon exchange between two constituent quarks and is the important source of the isospin symmetry breaking.

\section{THE HEAVY BARYON MASS}

In the framework of HBChPT, the correction to the self energy of the heavy baryons from the explicit flavor SU(3) breaking terms is $O\left(p^{2}\right)$. The one-loop chiral correction appears at $O\left(p^{3}\right)$.

\section{A. The Counterterms}

In the ChPT framework, the divergence from the loop diagram is absorbed by the counterterms at the same or lower orders. These counterterms come from Eq.(6). All the self-energy functions $\Sigma$ of counterterms are listed as follows:

$$
\begin{aligned}
\Sigma_{b c}\left(\Sigma_{c}^{0}\right) & =4 b_{1} B_{0} m_{d}+4 B_{0} c_{1} m_{d}+4 B_{0} c_{1} m_{s}+4 B_{0} c_{1} m_{u} \\
\Sigma_{b c}\left(\Xi_{c}^{\prime 0}\right) & =2 b_{1} B_{0} m_{d}+4 B_{0} c_{1} m_{d}+2 b_{1} B_{0} m_{s}+4 B_{0} c_{1} m_{s}+4 B_{0} c_{1} m_{u} \\
\Sigma_{b c}\left(\Omega_{c}^{0}\right) & =4 B_{0} c_{1} m_{d}+4 b_{1} B_{0} m_{s}+4 B_{0} c_{1} m_{s}+4 B_{0} c_{1} m_{u} \\
\Sigma_{b c}\left(\Sigma_{c}^{+}\right) & =2 b_{1} B_{0} m_{d}+4 B_{0} c_{1} m_{d}+4 B_{0} c_{1} m_{s}+2 b_{1} B_{0} m_{u}+4 B_{0} c_{1} m_{u} \\
\Sigma_{b c}\left(\Xi_{c}^{\prime+}\right) & =4 B_{0} c_{1} m_{d}+2 b_{1} B_{0} m_{s}+4 B_{0} c_{1} m_{s}+2 b_{1} B_{0} m_{u}+4 B_{0} c_{1} m_{u} \\
\Sigma_{b c}\left(\Sigma_{c}^{++}\right) & =4 B_{0} c_{1} m_{d}+4 B_{0} c_{1} m_{s}+4 b_{1} B_{0} m_{u}+4 B_{0} c_{1} m_{u} \\
\Sigma_{b c}\left(\Xi_{c}^{0}\right) & =4 b_{6} B_{0} m_{d}+8 B_{0} c_{6} m_{d}+4 b_{6} B_{0} m_{s}+8 B_{0} c_{6} m_{s}+8 B_{0} c_{6} m_{u} \\
\Sigma_{b c}\left(\Lambda_{c}^{+}\right) & =4 b_{6} B_{0} m_{d}+8 B_{0} c_{6} m_{d}+8 B_{0} c_{6} m_{s}+4 b_{6} B_{0} m_{u}+8 B_{0} c_{6} m_{u} \\
\Sigma_{b c}\left(\Xi_{c}^{+}\right) & =8 B_{0} c_{6} m_{d}+4 b_{6} B_{0} m_{s}+8 B_{0} c_{6} m_{s}+4 b_{6} B_{0} m_{u}+8 B_{0} c_{6} m_{u} \\
\Sigma_{b c}\left(\Sigma_{c}^{* 0}\right) & =-4 b_{5} B_{0} m_{d}-4 B_{0} c_{5} m_{d}-4 B_{0} c_{5} m_{s}-4 B_{0} c_{5} m_{u} \\
\Sigma_{b c}\left(\Xi_{c}^{* \prime 0}\right) & =-2 b_{5} B_{0} m_{d}-4 B_{0} c_{5} m_{d}-2 b_{5} B_{0} m_{s}-4 B_{0} c_{5} m_{s}-4 B_{0} c_{5} m_{u} \\
\Sigma_{b c}\left(\Omega_{c}^{* 0}\right) & =-4 B_{0} c_{5} m_{d}-4 b_{5} B_{0} m_{s}-4 B_{0} c_{5} m_{s}-4 B_{0} c_{5} m_{u} \\
\Sigma_{b c}\left(\Sigma_{c}^{*+}\right) & =-2 b_{5} B_{0} m_{d}-4 B_{0} c_{5} m_{d}-4 B_{0} c_{5} m_{s}-2 b_{5} B_{0} m_{u}-4 B_{0} c_{5} m_{u} \\
\Sigma_{b c}\left(\Xi_{c}^{* \prime+}\right) & =-4 B_{0} c_{5} m_{d}-2 b_{5} B_{0} m_{s}-4 B_{0} c_{5} m_{s}-2 b_{5} B_{0} m_{u}-4 B_{0} c_{5} m_{u} \\
\Sigma_{b c}\left(\Sigma_{c}^{*++}\right) & =-4 B_{0} c_{5} m_{d}-4 B_{0} c_{5} m_{s}-4 b_{5} B_{0} m_{u}-4 B_{0} c_{5} m_{u}
\end{aligned}
$$

The mass splitting of the isospin multiplets mainly arises from QED effects and the mass difference between up and down quarks. Light quarks have different charges. In fact, the tree-level QED correction starts at $O\left(p^{2}\right)$. They also act as the counterterms. All the self-energy functions $\Sigma$ of QED counterterms are listed as follows:

$$
\begin{aligned}
\Sigma_{\mathrm{QED}}\left(\Xi_{c}^{\prime 0}\right) & =4 e^{2} e_{3}^{66} \\
\Sigma_{\mathrm{QED}}\left(\Sigma_{c}^{0}\right) & =4 e^{2} e_{3}^{66} \\
\Sigma_{\mathrm{QED}}\left(\Omega_{c}^{0}\right) & =4 e^{2} e_{3}^{66} \\
\Sigma_{\mathrm{QED}}\left(\Xi_{c}^{\prime+}\right) & =2 e^{2} e_{1}^{66}+2 e^{2} e_{2}^{66}+4 e^{2} e_{3}^{66} \\
\Sigma_{\mathrm{QED}}\left(\Sigma_{c}^{+}\right) & =2 e^{2} e_{1}^{66}+2 e^{2} e_{2}^{66}+4 e^{2} e_{3}^{66} \\
\Sigma_{\mathrm{QED}}\left(\Sigma_{c}^{++}\right) & =4 e^{2} e_{1}^{66}+4 e^{2} e_{2}^{66}+4 e^{2} e_{3}^{6}+4 e^{2} e_{4}^{66} \\
\Sigma_{\mathrm{QED}}\left(\Xi_{c}^{0}\right) & =8 e^{2} e_{3}^{\overline{3} \overline{3}} \\
\Sigma_{\mathrm{QED}}\left(\Lambda_{c}^{+}\right) & =4 e^{2} e_{1}^{\overline{3} \overline{3}}+4 e^{2} e_{2}^{\overline{3} \overline{3}}+8 e^{2} e_{3}^{\overline{3} \overline{3}} \\
\Sigma_{\mathrm{QED}}\left(\Xi_{c}^{+}\right) & =4 e^{2} e_{1}^{\overline{3} \overline{3}}+4 e^{2} e_{2}^{\overline{3} \overline{3}}+8 e^{2} e_{3}^{\overline{3} \overline{3}}
\end{aligned}
$$




$$
\begin{aligned}
\Sigma_{\mathrm{QED}}\left(\Xi_{c}^{* \prime 0}\right) & =-4 e^{2} e_{3}^{6^{*} 6^{*}} \\
\Sigma_{\mathrm{QED}}\left(\Sigma_{c}^{* 0}\right) & =-4 e^{2} e_{3}^{6^{*} 6^{*}} \\
\Sigma_{\mathrm{QED}}\left(\Omega_{c}^{* 0}\right) & =-4 e^{2} e_{3}^{6^{*} 6^{*}} \\
\Sigma_{\mathrm{QED}}\left(\Xi_{c}^{* \prime+}\right) & =-2 e^{2} e_{1}^{6^{*} 6^{*}}-2 e^{2} e_{2}^{6^{*} 6^{*}}-4 e^{2} e_{3}^{6^{*} 6^{*}} \\
\Sigma_{\mathrm{QED}}\left(\Sigma_{c}^{*+}\right) & =-2 e^{2} e_{1}^{6^{*} 6^{*}}-2 e^{2} e_{2}^{6^{*} 6^{*}}-4 e^{2} e_{3}^{6^{*} 6^{*}} \\
\Sigma_{\mathrm{QED}}\left(\Sigma_{c}^{*++}\right) & =-4 e^{2} e_{1}^{6^{*} 6^{*}}-4 e^{2} e_{2}^{6^{*} 6^{*}}-4 e^{2} e_{3}^{6^{*} 6^{*}}-4 e^{2} e_{4}^{6^{*} 6^{*}}
\end{aligned}
$$

\section{B. Loop contribution}

The lowest order loop correction is $O\left(p^{3}\right)$ where the interaction vertex in Fig. 1 arises from $\hat{\mathcal{L}}^{(1)}$. The single line represents a spin- $\frac{1}{2}$ baryon and double line a spin- $\frac{3}{2}$ baryon. In the computation of the Feynman diagrams, we need the spin projection operators $P_{(33) \mu \nu}^{\frac{3}{2}}$ of the spin- $\frac{3}{2}$ heavy baryons 31 33]. Some properties of the spin projection operator and the Pauli-Lubanski spin operator $S^{\mu}$ are collected in Appendix VID.

The self-energy function can be written as

$$
\begin{aligned}
\Sigma_{6, \overline{3}, \mathrm{I}} & =C_{6, \overline{3}}\left(A_{6, \overline{3}, \mathrm{I}}+B_{6, \overline{3}, \mathrm{I}} \epsilon\right) f(m, \omega) \\
\Sigma_{6, \overline{3}, \mathrm{II}} & =C_{6, \overline{3}}\left(A_{6, \overline{3}, \mathrm{II}}+B_{6, \overline{3}, \mathrm{II}} \epsilon\right) f(m, \omega) \\
\Sigma_{6^{*}, \mathrm{I}} & =C_{6^{*}}\left(A_{6^{*}, \mathrm{I}}+B_{6^{*}, \mathrm{I}} \epsilon\right) f(m, \omega) \\
\Sigma_{6^{*}, \mathrm{II}} & =C_{6^{*}}\left(A_{6^{*}, \mathrm{II}}+B_{6^{*}, \mathrm{II}} \epsilon\right) f(m, \omega)
\end{aligned}
$$

The function $f(m, \omega)$ is defined in Appendix VIC The parameters $A$ and $B$ are related to the dimension $d=4-\epsilon$ in the dimensional regularization. For the spin- $\frac{1}{2}$ particles $A_{\mathrm{I}}+B_{\mathrm{I}} \epsilon=-\frac{1}{4}, A_{\mathrm{II}}+B_{\mathrm{II}} \epsilon=\frac{d-2}{d-1}$. For the spin- $\frac{3}{2}$ particles, $A_{\mathrm{I}}+B_{\mathrm{I}} \epsilon=\frac{-(d+1)(d-3)}{4(d-1)^{2}}, A_{\mathrm{II}}+B_{\mathrm{II}} \epsilon=\frac{1}{d-1}$. The coefficients $\mathrm{C}$ are listed in Table VII in the appendix.

FIG. 1: The one-loop Feynman diagrams that contribute to the self energy.

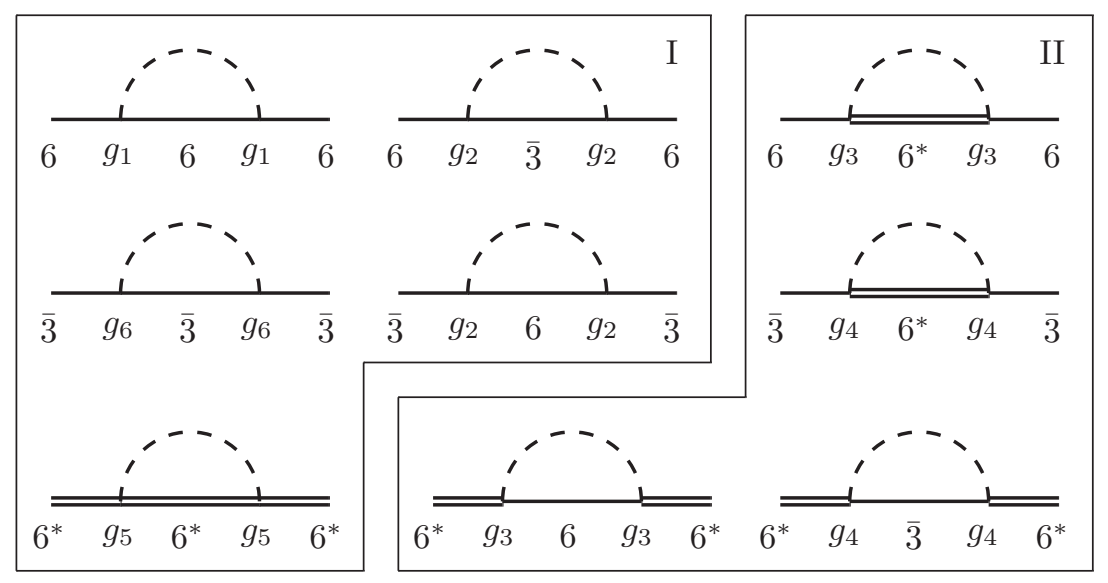

We calculate the loop contribution for each type of diagram listed in Fig. 1 separately. The intermediate and external baryons have the same spin for the type-I loops while their spin is different for the type-II loops. Throughout our calculation, we use the $\overline{\mathrm{MS}}$ (modified minimal subtraction) scheme. The simplest case corresponds to $\delta_{1}=\delta_{2}=0$, where the self-energy correction has a very simple form,

$$
\Sigma^{\text {loop }} \propto m_{\phi}^{3}
$$

where $m_{\phi}$ is the pseudoscalar meson mass.

The QED correction may also appear at $O\left(p^{3}\right)$ from the photon loop. With

$$
r_{\mu}=l_{\mu}=-Q \mathcal{A}_{\mu}
$$


where $Q$ is the charge operator and $\mathcal{A}_{\mu}$ represents the photon field, the chiral connection $\Gamma_{\mu}$ is modified as follows

$$
\begin{aligned}
\Gamma_{\mu} & =\frac{1}{2}\left(u^{\dagger} \partial_{\mu} u+u \partial_{\mu} u^{\dagger}\right)-\frac{i}{2}\left(u^{\dagger} r_{\mu} u+u l_{\mu} u^{\dagger}\right) \\
& =\Gamma_{\mu}^{0}+\Gamma_{\mu}^{r, l}
\end{aligned}
$$

At the lowest order

$$
\begin{gathered}
\Gamma_{\mu}^{\mathrm{QED}}=i \mathcal{A}_{\mu} Q+O\left(\phi^{2}\right) \\
\hat{\mathcal{L}}_{\mathrm{con}, \mathrm{QED}}=-v^{\mu} \mathcal{A}_{\mu} t r \overline{\mathcal{N}}\left(Q \mathcal{N}+\mathcal{N} Q^{T}\right)
\end{gathered}
$$

The correction to the self energy from the following photon loop vanishes with the infrared regularization as pointed out in Ref. 34].

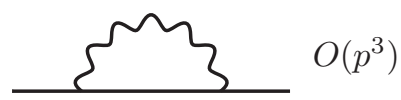

The charge operator may also enter $u_{\mu}$.

$$
\begin{aligned}
u_{\mu} & =\frac{i}{2}\left(u^{\dagger} \partial_{\mu} u-u \partial_{\mu} u^{\dagger}\right)+\frac{i}{2}\left[u^{\dagger}\left(-i r_{\mu}\right) u-u\left(-i l_{\mu}\right) u^{\dagger}\right] \\
& =u_{\mu}^{0}+u_{\mu}^{r, l}
\end{aligned}
$$

However, its contribution is of higher order. Numerically, the QED effects are very small since the QED Lagrangian can be expanded both in terms of the chiral order and the fine-structure constant $\alpha$, which is also a small number. If we calculate the correction up to $O\left(p^{4}\right)$, the higher order of $\alpha$ should also be considered, whose contributions will be much smaller.

To sum up, the heavy baryon mass reads

$$
M=\stackrel{\circ}{M}+\Sigma_{b c}+\Sigma_{\mathrm{QED}}+\Sigma^{\mathrm{loop}}
$$

where $\stackrel{\circ}{M}$ is the bare mass without the chiral corrections.

\section{Numerical results}

In our numerical analysis, the LECs $b$ and $c$ are replaced by the dimensionless parameters $b^{\prime}$ and $c^{\prime}$, which are defined in Eqs. (16)-(17),

$$
\begin{aligned}
& b_{1,5}^{\prime}=B_{0} b_{1,5}, \quad c_{1,5}^{\prime}=B_{0} c_{1,5}+\frac{\stackrel{\circ}{M}_{6,6^{*}}}{4\left(m_{u}+m_{d}+m_{s}\right)} \\
& b_{6}^{\prime}=B_{0} b_{6}, \quad c_{6}^{\prime}=B_{0} c_{6}+\frac{\stackrel{\circ}{M}_{\overline{3}}}{8\left(m_{u}+m_{d}+m_{s}\right)}
\end{aligned}
$$

With the experimental values of the heavy baryon masses as input [5], we extract the values of the LECs $b^{\prime} \mathrm{s}$ and $c^{\prime}$ s. Fit 1 corresponds to the case of including the type-I loop corrections only. $b_{1}^{\prime}=-1.69, c_{1}^{\prime}=-6.27, b_{6}^{\prime}=-1.12, c_{6}^{\prime}=$ $-2.71, b_{5}^{\prime}=-1.65, c_{5}^{\prime}=-6.30$. Fit 2 contains both the type-I and type-II loop corrections. $b_{1}^{\prime}=-2.10, c_{1}^{\prime}=$ $-6.49, b_{6}^{\prime}=-2.43, c_{6}^{\prime}=-2.91, b_{5}^{\prime}=-2.11, c_{5}^{\prime}=-6.64$. We also list the contribution of the type-I loop and sum of type-I and type-II loops in the second to fourth columns of Table 1 explicitly. The heavy baryon masses with the notation $\ddagger$ in the fourth to fifth columns are the predicted values. The errors are also listed in the table. Some of them are numerically very small, which are omitted in the table. The mass splitting between the spin- $\frac{1}{2}$ charmed baryons was also discussed in Ref. [34].

The LECs at $O\left(p^{2}\right)$ were estimated in Refs. [8, 9], where the $\mathrm{SU}(3)$ flavor symmetry-breaking Lagrangian reads

$$
\mathcal{L}^{(2)}=c_{1} \operatorname{tr} \bar{B}_{6} \tilde{\chi}_{+} B_{6}+\bar{c}_{1} \operatorname{tr} \bar{B}_{\overline{3}} \tilde{\chi}_{+} B_{\overline{3}}+\cdots
$$


TABLE I: Chiral loop corrections to the heavy baryon masses in unit of $\mathrm{MeV}$ with $\delta=0$.

The marked entries are the predictions.

\begin{tabular}{|c|c|c|c|c|c|c|}
\hline & \multicolumn{3}{|c|}{ Loop contribution } & \multicolumn{3}{|c|}{ Heavy Baryon Masses } \\
\hline & case I & case II & case I+II & Experimental data & Fit 1 for case I & Fit 2 for case $\mathrm{I}+\mathrm{II}$ \\
\hline$M_{\Sigma_{c}^{++}}$ & -305.00 & -100.87 & -405.87 & $2454.02 \pm 0.18$ & $2449.04 \pm 2.1^{\ddagger}$ & $2448.38 \pm 2.1^{\ddagger}$ \\
\hline$M_{\Sigma_{c}^{+}}$ & -307.91 & -101.83 & -409.74 & $2452.9 \pm 0.4$ & $2452.90 \pm 0.4$ & $2452.90 \pm 0.4$ \\
\hline$M_{\Sigma_{c}^{0}}$ & -310.68 & -102.50 & -413.18 & $2453.76 \pm 0.18$ & $2456.90 \pm 2.1^{\ddagger}$ & $2457.85 \pm 2.1^{\ddagger}$ \\
\hline$M_{\Xi_{c}^{\prime+}}$ & -512.58 & -177.86 & -690.44 & $2575.6 \pm 3.1$ & $2573.14 \pm 2.1^{\ddagger}$ & $2574.83 \pm 2.1^{\ddagger}$ \\
\hline$M_{\Xi_{c}^{\prime 0}}$ & -516.23 & -180.30 & -696.53 & $2577.9 \pm 2.9$ & $2576.25 \pm 2.1^{\ddagger}$ & $2577.13 \pm 2.1^{\ddagger}$ \\
\hline$M_{\Omega_{c}^{0}}^{c}$ & -722.19 & -258.91 & -981.09 & $2695.2 \pm 1.7$ & $2695.20 \pm 1.7$ & $2695.20 \pm 1.7$ \\
\hline$M_{\Lambda_{c}^{+}}$ & -108.81 & -217.63 & -326.44 & $2286.46 \pm 0.14$ & $2286.46 \pm 0.14$ & $2286.46 \pm 0.14$ \\
\hline$M_{\Xi_{c}^{+}}^{\Lambda_{c}}$ & -358.79 & -717.57 & -1076.36 & $2467.8_{-0.6}^{+0.4}$ & $2467.80_{-0.6}^{+0.4}$ & $2467.80_{-0.6}^{+0.4}$ \\
\hline$M_{\Xi_{c}^{0}}$ & -362.44 & -724.89 & -1087.33 & $2470.88_{-0.80}^{+0.34}$ & $2473.14 \pm 0.65^{\ddagger}$ & $2476.23 \pm 0.65^{\ddagger}$ \\
\hline$\overline{M_{\Sigma_{c}^{*++}}}$ & -252.18 & -153.69 & -405.87 & $2518.4 \pm 0.6$ & $2513.29 \pm 4.3^{\ddagger}$ & $2512.92 \pm 4.3^{\mp}$ \\
\hline$M_{\Sigma_{c}^{*+}}$ & -254.58 & -155.17 & -409.74 & $2517.5 \pm 2.3$ & $2517.50 \pm 2.3$ & $2517.50 \pm 2.3$ \\
\hline$M_{\Sigma_{c}^{* 0}}$ & -256.25 & -156.93 & -413.18 & $2518.0 \pm 0.5$ & $2522.44 \pm 4.3^{\ddagger}$ & $2522.51 \pm 4.3^{\ddagger}$ \\
\hline$M_{\Xi_{c}^{\prime *+}}^{c}$ & -444.65 & -245.78 & -690.44 & $2645.9_{-0.6}^{+0.5}$ & $2644.76 \pm 4.3^{\ddagger}$ & $2642.45 \pm 4.3^{\ddagger}$ \\
\hline$M_{\Xi_{c}^{\prime * 0}}$ & -450.75 & -245.78 & -696.53 & $2645.9 \pm 0.5$ & $2645.18 \pm 4.3^{\ddagger}$ & $2644.82 \pm 4.3^{\ddagger}$ \\
\hline$M_{\Omega_{c}^{* 0}}^{c}$ & -647.27 & -333.83 & -981.10 & $2765.9 \pm 2.0$ & $2765.90 \pm 2.0$ & $2765.90 \pm 2.0$ \\
\hline $\begin{array}{l}b_{1}^{\prime} \\
c_{1}^{\prime} \\
\end{array}$ & & & & & $\begin{array}{c}-1.69 \\
-6.27 \pm 4.8 \times 10^{-3} \\
\end{array}$ & $\begin{array}{c}-2.10 \\
-6.49 \pm 4.8 \times 10^{-3}\end{array}$ \\
\hline $\begin{array}{l}b_{6}^{\prime} \\
c_{6}^{\prime} \\
\end{array}$ & & & & & $\begin{array}{c}-1.12 \\
-2.71 \pm 0.7 \times 10^{-3} \\
\end{array}$ & $\begin{array}{c}-2.43 \\
-2.91 \pm 0.7 \times 10^{-3}\end{array}$ \\
\hline $\begin{array}{l}b_{5}^{\prime} \\
c_{5}^{\prime}\end{array}$ & & & & & $\begin{array}{c}-1.65 \\
-6.30 \pm 9.9 \times 10^{-3} \\
\end{array}$ & $\begin{array}{c}-2.11 \\
-6.64 \pm 9.9 \times 10^{-3}\end{array}$ \\
\hline
\end{tabular}

with

$$
\tilde{\chi}_{+}=\chi_{+}-\frac{1}{3} \operatorname{tr} \chi_{+}
$$

The authors first constructed the flavor SU(4) Lagrangian. Then they reduced the SU(4) Lagrangian into the SU(3) form. In this way, they estimated the LECs. Using the Gell-Mann-Okubo relation, the LECs extracted in Refs. [8, 9] correspond to the following values of $b$ 's

$$
\begin{gathered}
b_{1,5}^{\prime}=B_{0} b_{1,5} \approx-2.30 \\
b_{6}^{\prime}=B_{0} b_{6} \approx-1.22
\end{gathered}
$$

These values are consistent with the above values extracted from fitting to experimental data in this work.

The spin and flavor representation of the external and intermediate baryons may be different. Their mass splitting will contribute to the self energy through the chiral loop. Such corrections are quite important in the nucleon octet and $\Delta$ decuplet case. We consider three cases. Fit 3 corresponds to the case when the type-I loop correction is included with $\delta \neq 0$. Fit 4 includes both types of loop corrections with $\delta \neq 0$. Fit 5 corresponds to the inclusion of both types of loop corrections with $\delta \neq 0$ and QED effects.

We collect the fit results Table Comparing the fourth column in Table \I we notice that the loop contributions are suppressed after considering the mass difference $\delta$. On the other hand, the absolute value of parameters $b$ and $c$ becomes slightly smaller. $b_{1}^{\prime}=-2.05, c_{1}^{\prime}=-6.44, b_{6}^{\prime}=-2.37, c_{6}^{\prime}=-2.87, b_{5}^{\prime}=-1.95, c_{5}^{\prime}=-6.50$.

Even if the QED effects are not considered, the up and down quark mass difference will cause the isospin breaking. The experimental baryon masses always contain the isospin breaking. When we consider QED corrections, more LECs contribute to the self energy. The LECs $e_{3}^{66}, e_{3}^{6^{*}} 6^{*}, e_{3}^{\overline{3} \overline{3}}$ can be absorbed by $c_{1}^{\prime}, c_{5}^{\prime}, c_{6}^{\prime}$.

$$
\begin{gathered}
c_{1,5}^{\prime}=B_{0} c_{1,5}+\frac{\stackrel{\circ}{M}_{6,6^{*}}+e^{2} e_{3}^{66,6^{*} 6^{*}}}{4\left(m_{u}+m_{d}+m_{s}\right)} \\
c_{6}^{\prime}=B_{0} c_{6}+\frac{\stackrel{\circ}{M}_{\overline{3}}+e^{2} e_{3}^{\overline{3} \overline{3}}}{8\left(m_{u}+m_{d}+m_{s}\right) .}
\end{gathered}
$$


In this case, $b_{1}^{\prime}=-2.06, c_{1}^{\prime}=-6.43, b_{6}^{\prime}=-2.37, c_{6}^{\prime}=-2.86, b_{5}^{\prime}=-1.97, c_{5}^{\prime}=-6.49$. The values of $e^{2}\left(e_{1}+e_{2}\right)$ and $e^{2} e_{4}$ are given in Table II] The heavy baryon masses with $\ddagger$ in the fifth to seventh columns are the predicted values.

In the isospin symmetry limit, the divergences from the loop diagrams can be absorbed by the LECs (or counterterm) at $O\left(p^{2}\right)$. However, the low energy constants at $O\left(p^{2}\right)$ are not enough to cancel the divergences from the loop diagrams at $O\left(p^{3}\right)$ when we consider the SU(2)-breaking corrections, which was also pointed out in Ref. 34. Chiral symmetry ensures that the divergences will be absorbed by the counterterms at the higher order if we treat the $\mathrm{SU}(2)$ symmetrybreaking terms as the higher order correction. In Ref. 34], the authors studied the spin- $\frac{1}{2}$ baryons. In our work, we studied both the spin- $\frac{1}{2}$ and $-\frac{3}{2}$ heavy baryons. In Ref. [34], the authors focused on the mass splitting only and used the experimental mass splitting as input with the infrared regularization scheme. In our work, we calculated all the possible chiral corrections to the heavy baryon masses up to $O\left(p^{3}\right)$ and used the values of experimental mass as input within the framework of heavy baryon ChPT.

TABLE II: Chiral loop corrections to the heavy baryon masses in units of MeV with $\delta \neq 0$ and QED effects.

The marked entries are the predictions.

\begin{tabular}{|c|c|c|c|c|c|c|}
\hline & \multicolumn{3}{|c|}{ Loop contribution $\Sigma^{\text {loop }}$} & \multicolumn{3}{|c|}{ Mass of baryons } \\
\hline & case I & case II & case I+II & Fit 3 for case I & Fit 4 for case I+II & Fit 5 for case I+II with QED \\
\hline$M_{\Sigma_{c}^{++}}$ & -278.36 & -103.34 & -381.70 & $2449.56 \pm 2.1^{\mp}$ & $2448.91 \pm 2.1^{\mp}$ & $2454.02 \pm 0.18$ \\
\hline$M_{\Sigma_{c}^{+}}^{\Sigma_{c}}$ & -281.59 & -104.32 & -385.91 & $2452.90 \pm 0.4$ & $2452.90 \pm 0.4$ & $2452.90 \pm 0.4$ \\
\hline$M_{\Sigma_{c}^{0}}$ & -283.95 & -104.93 & -388.88 & $2457.11 \pm 2.1^{\ddagger}$ & $2458.13 \pm 2.1^{\ddagger}$ & $2453.76 \pm 0.18$ \\
\hline$M_{\Xi_{c}^{\prime+}}$ & -480.54 & -181.43 & -661.97 & $2569.42 \pm 2.1^{\ddagger}$ & $2570.48 \pm 2.1^{\ddagger}$ & $2572.66 \pm 2.46^{\ddagger}$ \\
\hline$M_{\Xi_{c}^{\prime 0}}$ & -484.24 & -183.82 & -668.06 & $2572.30 \pm 2.1^{\ddagger}$ & $2572.59 \pm 2.1^{\ddagger}$ & $2570.40 \pm 2.46^{\ddagger}$ \\
\hline$M_{\Omega_{c}^{0}}^{c}$ & -676.81 & -262.29 & -939.09 & $2695.20 \pm 1.7$ & $2695.20 \pm 1.7$ & $2695.20 \pm 1.7$ \\
\hline 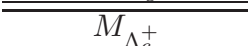 & -111.09 & -177.57 & -288.66 & $2286.46 \pm 0.14$ & $22286.46 \pm 0.14$ & $2286.46 \pm 0.14$ \\
\hline$M_{\Xi_{c}^{+}}^{\Lambda_{c}}$ & -367.68 & -650.46 & -1018.14 & $2467.80_{-0.6}^{+0.4}$ & $2467.80_{-0.6}^{+0.4}$ & $2467.80_{-0.6}^{+0.4}$ \\
\hline$M_{\Xi_{c}^{0}}$ & -371.24 & -657.02 & -1028.26 & $2473.36 \pm 0.65^{\ddagger}$ & $2476.66 \pm 0.65^{\ddagger}$ & $2470.88_{-0.80}^{+0.38}$ \\
\hline$\overline{M_{\Sigma^{*++}}}$ & -252.18 & $\overline{-92.06}$ & -344.25 & $2513.29 \pm 4.3^{\mp}$ & $2513.88 \pm 4.3^{\mp}$ & $2518.40 \pm 0.6$ \\
\hline$M_{\Sigma_{c}^{*+}}$ & -254.58 & -93.87 & -348.45 & $2517.50 \pm 2.3$ & $2517.50 \pm 2.3$ & $2517.50 \pm 2.3$ \\
\hline$M_{\Sigma_{c}^{* 0}}^{L_{c}}$ & -256.25 & -94.84 & -351.09 & $2522.44 \pm 4.3^{\ddagger}$ & $2522.68 \pm 4.3^{\ddagger}$ & $2518.0 \pm 0.5$ \\
\hline$M_{\Xi_{c}^{\prime *+}}$ & -444.65 & -161.97 & -606.63 & $2644.67 \pm 4.3^{\ddagger}$ & $2634.50 \pm 4.3^{\ddagger}$ & $2636.83 \pm 5.4^{\ddagger}$ \\
\hline $\overrightarrow{M_{\Xi^{\prime * 0}}}$ & -450.75 & -162.15 & -612.89 & $2645.18 \pm 4.3^{\ddagger}$ & $2636.05 \pm 4.3^{\ddagger}$ & $2633.71 \pm 5.4^{\ddagger}$ \\
\hline$M_{\Omega_{c}^{* 0}}^{c}$ & -647.27 & -210.96 & -858.22 & $2765.90 \pm 2.0$ & $2765.90 \pm 2.0$ & $2765.90 \pm 2.0$ \\
\hline $\begin{array}{c}b_{1}^{\prime} \\
c_{1}^{\prime} \\
e^{2}\left(e_{1}^{66}+e_{2}^{66}\right) \\
e^{2} e_{4}^{66}\end{array}$ & & & & $\begin{array}{c}-1.64 \\
-6.21 \pm 4.8 \times 10^{-3}\end{array}$ & $\begin{array}{c}-2.05 \\
-6.44 \pm 4.8 \times 10^{-3}\end{array}$ & $\begin{aligned} & -2.06 \\
-6.43 & \pm 5.6 \times 10^{-3} \\
& -2.21 \\
& -0.19\end{aligned}$ \\
\hline $\begin{array}{c}b_{6}^{\prime} \\
c_{6}^{\prime} \\
e^{2}\left(e_{1}^{\overline{3} \overline{3}}+e_{2}^{\overline{3} \overline{3}}\right) \\
\end{array}$ & & & & $\begin{array}{c}-1.14 \\
-2.71 \pm 0.7 \times 10^{-3}\end{array}$ & $\begin{array}{c}-2.37 \\
-2.87 \pm 0.7 \times 10^{-3}\end{array}$ & $\begin{array}{c}-2.37 \\
-2.86 \pm 1.4 \times 10^{-3} \\
-1.44 \\
\end{array}$ \\
\hline $\begin{array}{c}b_{5}^{\prime} \\
c_{5}^{\prime} \\
e^{2}\left(e_{1}^{6^{*} 6^{*}}+e_{2}^{6^{*} 6^{*}}\right) \\
e^{2} e_{4}^{6^{*} 6^{*}}\end{array}$ & & & & $\begin{array}{c}-1.65 \\
-6.30 \pm 9.9 \times 10^{-3}\end{array}$ & $\begin{array}{c}-1.95 \\
-6.50 \pm 9.9 \times 10^{-3}\end{array}$ & $\begin{array}{c}-1.97 \\
-6.49 \pm 0.0124 \\
-2.36 \\
0.04\end{array}$ \\
\hline
\end{tabular}

TABLE III: The decay width of the heavy baryons in units of MeV.

\begin{tabular}{cccc}
\hline \multicolumn{4}{c}{ Experimental data case I loop case II loop } \\
\hline$\Gamma_{\Sigma_{c}^{++}}$ & 2.23 & 2.60 & \\
$\Gamma_{\Sigma_{c}^{+}}$ & $<4.6$ & 3.20 & \\
$\Gamma_{\Sigma_{c}^{0}}$ & 2.2 & 2.60 & \\
\hline$\Gamma_{\Sigma_{c}^{*++}}$ & 14.9 & 8.10 \\
$\Gamma_{\Sigma_{c}^{*+}}$ & $<17$ & 8.96 \\
$\Gamma_{\Sigma_{c}^{* 0}}$ & 16.1 & 8.10 \\
$\Gamma_{\Xi_{c}^{\prime *+}}^{\prime+}$ & $<3.1$ & 6.28 \\
$\Gamma_{\Xi_{c}^{\prime * 0}}$ & $<5.5$ & 6.28 \\
\hline
\end{tabular}


In the above analysis, $\delta$ is the difference of the average mass between baryons in the different representations. In the derivation of the imaginary part of the loop diagrams, one should be cautious about the choice of $\delta$. For example, the process $\Sigma_{c} \rightarrow \Lambda_{c}^{+}+\pi$ is forbidden if we choose the average value: $\delta_{\Sigma_{c} \Lambda_{c}^{+}}=M_{\Sigma_{c}}-M_{\Lambda_{c}^{+}}=126.52 \mathrm{MeV}<M_{\pi}$. To avoid such a paradox, we used the experimental mass as input to calculate $\delta_{\Sigma_{c} \Lambda_{c}^{+}}$and the imaginary part of the self energy. For all the other processes, $\delta$ takes the average value. We collect the experimental and theoretical width $\Gamma$ in Table III. These values are consistent with experimental data.

\section{THE AXIAL CHARGE OF THE HEAVY BARYON}

The baryon axial charge is a very important physical observable, which can be measured through semileptonic decays. In this section, we will explore the chiral corrections to the axial charges $g_{1}$ to $g_{6}$ in Eq. (4). At the leading order, the axial currents are determined by chiral symmetry entirely. At $O\left(p^{2}\right)$, the loop contributions arise from the vertex correction and wave function renormalization while the correction from the chiral connection vanishes in the heavy quark limit $M_{c} \rightarrow \infty$.

\section{A. The axial currents on tree level}

The axial currents at the tree level can be obtained from Eq. (5). With the external source $r^{\mu}, l^{\mu}$ in Eqs. (12) and (14)

$$
r_{\mu}=\frac{\lambda^{a}}{2} r_{\mu}^{a}, \quad l_{\mu}=\frac{\lambda^{a}}{2} l_{\mu}^{a}
$$

where $\lambda^{a}$ is the Gell-Mann generator in the flavor space, the difference of the chiral currents $R^{a, \mu}$ and $L^{a, \mu}$ leads to the axial current

$$
A^{a, \mu}=R^{a, \mu}-L^{a, \mu}
$$

The axial currents arising from the chiral connection and $O(p)$ interaction terms are

$$
\begin{aligned}
& A_{\text {con }}^{a, \mu}(\overline{3})= \frac{1}{8} v^{\mu} \operatorname{tr}\left[\overline{\mathcal{N}}_{\overline{3}}\left(u^{\dagger} \lambda^{a} u-u \lambda^{a} u^{\dagger}\right) \mathcal{N}_{\overline{3}}+\overline{\mathcal{N}}_{\overline{3}} \mathcal{N}_{\overline{3}}\left(u^{\dagger} \lambda^{a} u-u \lambda^{a} u^{\dagger}\right)^{T}\right] \\
& A_{\text {con }}^{a, \mu}(6)= \frac{1}{4} v^{\mu} \operatorname{tr}\left[\overline{\mathcal{N}}_{6}\left(u^{\dagger} \lambda^{a} u-u \lambda^{a} u^{\dagger}\right) \mathcal{N}_{6}+\overline{\mathcal{N}}_{6} \mathcal{N}_{6}\left(u^{\dagger} \lambda^{a} u-u \lambda^{a} u^{\dagger}\right)^{T}\right] \\
& A_{\text {con }}^{a, \mu}\left(6^{*}\right)= \frac{1}{4} v^{\mu} \operatorname{tr}\left\{-g_{\rho \sigma}\left[\overline{\mathcal{T}}^{\rho}\left(u^{\dagger} \lambda^{a} u-u \lambda^{a} u^{\dagger}\right) \mathcal{T}^{\sigma}+\overline{\mathcal{T}}^{\rho} \mathcal{T}^{\sigma}\left(u^{\dagger} \lambda^{a} u-u \lambda^{a} u^{\dagger}\right)^{T}\right]\right\} \\
& A^{a, \mu}\left(g_{1}\right)=\frac{1}{2} g_{1} \operatorname{tr}\left[\overline{\mathcal{N}}_{6} S^{\mu}\left(u^{\dagger} \lambda^{a} u+u \lambda^{a} u^{\dagger}\right) \mathcal{N}_{6}\right] \\
& A^{a, \mu}\left(g_{2}\right)=\frac{1}{2} g_{2} \operatorname{tr}\left[\overline{\mathcal{N}}_{6} S^{\mu}\left(u^{\dagger} \lambda^{a} u+u \lambda^{a} u^{\dagger}\right) \mathcal{N}_{\overline{3}}+h . c .\right] \\
& A^{a, \mu}\left(g_{3}\right)=\frac{1}{4} g_{3} \operatorname{tr}\left[\overline{\mathcal{T}}^{\mu}\left(u^{\dagger} \lambda^{a} u+u \lambda^{a} u^{\dagger}\right) \mathcal{N}_{6}+h . c .\right] \\
& A^{a, \mu}\left(g_{4}\right)=\frac{1}{4} g_{4} \operatorname{tr}\left[\overline{\mathcal{T}}^{\mu}\left(u^{\dagger} \lambda^{a} u+u \lambda^{a} u^{\dagger}\right) \mathcal{N}_{\overline{3}}+h . c .\right] \\
& A^{a, \mu}\left(g_{5}\right)=\frac{1}{2} g_{5} \operatorname{tr}\left[\overline{\mathcal{T}}^{\nu} S^{\mu}\left(u^{\dagger} \lambda^{a} u+u \lambda^{a} u^{\dagger}\right) \mathcal{T}_{\nu}\right]
\end{aligned}
$$

The lowest order axial charges arising from the $g_{1}-g_{5}$ terms of the sextet and antitriplet are collected in Table IV. where we only list the channels allowing the semileptonic decays.

The $O\left(p^{0}\right)$ axial current arises from the $O(p)$ Lagrangian. The $O\left(p^{3}\right) \mathrm{SU}(3)$ symmetry-breaking Lagrangian $\mathcal{L}_{\text {counter }}^{(3)}$ contributes to the $O\left(p^{2}\right)$ corrections to the axial current. Moreover, these new vertices will cancel the infinity from 
TABLE IV: The axial charge $g_{(i j)}^{(0)}$ at the tree level.

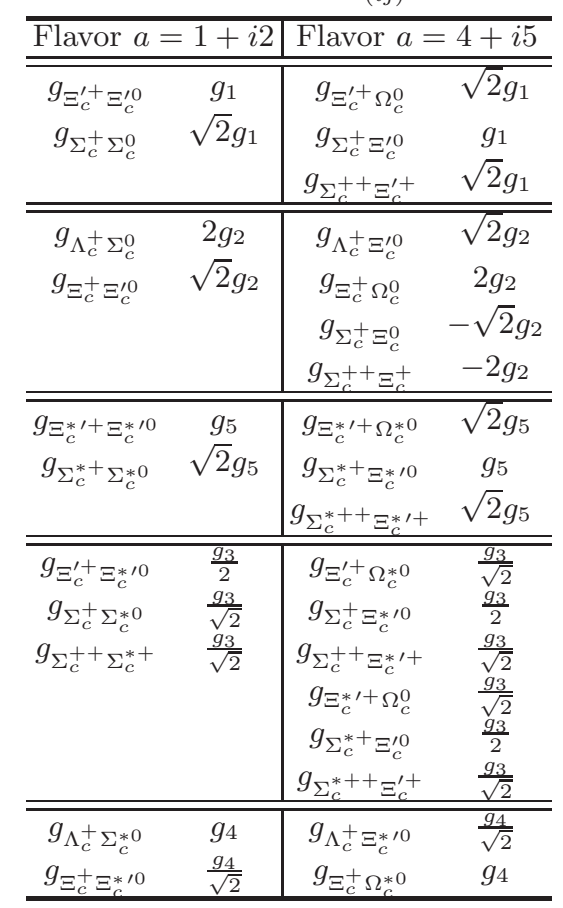

the loop corrections.

$$
\begin{aligned}
& \mathcal{L}_{\text {counter }}^{(3)} \\
= & d_{1} \operatorname{tr}\left(\bar{B}_{6} \gamma^{\mu} \gamma_{5}\left\{u_{\mu}, \chi_{+}\right\} B_{6}\right)+f_{1} \operatorname{tr}\left(\bar{B}_{6} \gamma^{\mu} \gamma_{5} u_{\mu} B_{6} \chi_{+}^{T}\right)+h_{1} \operatorname{tr}\left(\bar{B}_{6} \gamma^{\mu} \gamma_{5} u_{\mu} B_{6}\right) \operatorname{tr} \chi_{+} \\
& +d_{2} \operatorname{tr}\left(\bar{B}_{6} \gamma^{\mu} \gamma_{5}\left\{u_{\mu}, \chi_{+}\right\} B_{\overline{3}}\right)+f_{2} \operatorname{tr}\left(\bar{B}_{6} \gamma^{\mu} \gamma_{5} u_{\mu} B_{\overline{3}} \chi_{+}^{T}\right)+h_{2} \operatorname{tr}\left(\bar{B}_{6} \gamma^{\mu} \gamma_{5} u_{\mu} B_{\overline{3}}\right) \operatorname{tr} \chi_{+}+\text {h.c. } \\
& +d_{6} \operatorname{tr}\left(\bar{B}_{\overline{3}} \gamma^{\mu} \gamma_{5}\left\{u_{\mu}, \chi_{+}\right\} B_{\overline{3}}\right)+f_{6} \operatorname{tr}\left(\bar{B}_{\overline{3}} \gamma^{\mu} \gamma_{5} u_{\mu} B_{\overline{3}} \chi_{+}^{T}\right)+h_{6} \operatorname{tr}\left(\bar{B}_{\overline{3}} \gamma^{\mu} \gamma_{5} u_{\mu} B_{\overline{3}}\right) \operatorname{tr} \chi_{+} \\
& +d_{5} g_{\rho \sigma} \operatorname{tr}\left(\bar{B}_{6}^{* \rho} \gamma^{\mu} \gamma_{5}\left\{u_{\mu}, \chi_{+}\right\} B_{6}^{* \sigma}\right)+f_{5} g_{\rho \sigma} \operatorname{tr}\left(\bar{B}_{6}^{* \rho} \gamma^{\mu} \gamma_{5} u_{\mu} B_{6}^{* \sigma} \chi_{+}^{T}\right)+h_{5} g_{\rho \sigma} \operatorname{tr}\left(\bar{B}_{6}^{* \rho} \gamma^{\mu} \gamma_{5} u_{\mu} B_{6}^{* \sigma}\right) \operatorname{tr} \chi_{+} \\
& +d_{3} \operatorname{tr}\left(\bar{B}_{6}^{* \mu}\left\{u_{\mu}, \chi_{+}\right\} B_{6}\right)+f_{3} \operatorname{tr}\left(\bar{B}_{6}^{* \mu} u_{\mu} B_{6} \chi_{+}^{T}\right)+h_{3} \operatorname{tr}\left(\bar{B}_{6}^{* \mu} u_{\mu} B_{6}\right) \operatorname{tr} \chi_{+}+\text {h.c. } \\
& +d_{4} \operatorname{tr}\left(\bar{B}_{6}^{* \mu}\left\{u_{\mu}, \chi_{+}\right\} B_{\overline{3}}\right)+f_{4} \operatorname{tr}\left(\bar{B}_{6}^{* \mu} u_{\mu} B_{\overline{3}} \chi_{+}^{T}\right)+h_{4} \operatorname{tr}\left(\bar{B}_{6}^{* \mu} u_{\mu} B_{\overline{3}}\right) \operatorname{tr} \chi_{+}+\text {h.c. }
\end{aligned}
$$

In HBChPT, the $O\left(p^{2}\right)$ axial current arising from $\mathcal{L}_{\text {counter }}^{(3)}$ is

$$
\begin{aligned}
A_{d f h}^{a, \mu}= & \frac{d_{1}}{2} \operatorname{tr}\left(\overline{\mathcal{N}}_{6} S^{\mu}\left\{w_{+}^{a}, \chi_{+}\right\} \mathcal{N}_{6}\right)+\frac{f_{1}}{2} \operatorname{tr}\left(\overline{\mathcal{N}}_{6} S^{\mu} w_{+}^{a} \mathcal{N}_{6} \chi_{+}^{T}\right)+\frac{h_{1}}{2} \operatorname{tr}\left(\overline{\mathcal{N}}_{6} S^{\mu} w_{+}^{a} \mathcal{N}_{6}\right) \operatorname{tr} \chi_{+} \\
& +\frac{d_{2}}{2} \operatorname{tr}\left(\overline{\mathcal{N}}_{6} S^{\mu}\left\{w_{+}^{a}, \chi_{+}\right\} \mathcal{N}_{\overline{3}}\right)+\frac{f_{2}}{2} \operatorname{tr}\left(\overline{\mathcal{N}}_{6} S^{\mu} w_{+}^{a} \mathcal{N}_{\overline{3}} \chi_{+}^{T}\right)+\frac{h_{2}}{2} \operatorname{tr}\left(\overline{\mathcal{N}}_{6} S^{\mu} w_{+}^{a} \mathcal{N}_{\overline{3}}\right) \operatorname{tr} \chi_{+}+h . c . \\
& +\frac{d_{5}}{2} g_{\rho \sigma} \operatorname{tr}\left(\overline{\mathcal{T}}^{\rho} S^{\mu}\left\{w_{+}^{a}, \chi_{+}\right\} \mathcal{T}^{\sigma}\right)+\frac{f_{5}}{2} g_{\rho \sigma} \operatorname{tr}\left(\overline{\mathcal{T}}^{\rho} S^{\mu} w_{+}^{a} \mathcal{T}^{\sigma} \chi_{+}^{T}\right)+\frac{h_{5}}{2} g_{\rho \sigma} \operatorname{tr}\left(\overline{\mathcal{T}}^{\rho} S^{\mu} w_{+}^{a} \mathcal{T}^{\sigma}\right) \operatorname{tr} \chi_{+} \\
& +\frac{d_{3}}{4} \operatorname{tr}\left(\overline{\mathcal{T}}^{\mu}\left\{w_{+}^{a}, \chi_{+}\right\} \mathcal{N}_{6}\right)+\frac{f_{3}}{4} \operatorname{tr}\left(\overline{\mathcal{T}}^{\mu} w_{+}^{a} \mathcal{N}_{6} \chi_{+}^{T}\right)+\frac{h_{3}}{4} \operatorname{tr}\left(\overline{\mathcal{T}}^{\mu} w_{+}^{a} \mathcal{N}_{6}\right) \operatorname{tr} \chi_{+}+\text {h.c. } \\
& +\frac{d_{4}}{4} \operatorname{tr}\left(\overline{\mathcal{T}}^{\mu}\left\{w_{+}^{a}, \chi_{+}\right\} \mathcal{N}_{\overline{3}}\right)+\frac{f_{4}}{4} \operatorname{tr}\left(\overline{\mathcal{T}}^{\mu} w_{+}^{a} \mathcal{N}_{\overline{3}} \chi_{+}^{T}\right)+\frac{h_{4}}{4} \operatorname{tr}\left(\overline{\mathcal{T}}^{\mu} w_{+}^{a} \mathcal{N}_{\overline{3}}\right) \operatorname{tr} \chi_{+}+\text {h.c. }
\end{aligned}
$$

where

$$
w_{ \pm}^{a}=u^{\dagger} \lambda^{a} u \pm u \lambda^{a} u^{\dagger}
$$

The axial charges $g_{(i j)}^{(2)}$ in terms of the coefficients $d, f, h$ are listed in Table $\mathrm{V}$ 
TABLE V: The axial charges $g_{(i j)}^{(2)}$ from the counterterms.

\begin{tabular}{|c|c|c|}
\hline & $a=1+i 2$ & Flavor $a=4+i 5$ \\
\hline $\begin{array}{l}g_{\Xi_{c}^{\prime+} \Xi_{c}^{\prime 0}} \\
g_{\Sigma_{c}^{+} \Sigma_{c}^{0}}\end{array}$ & $\begin{array}{c}2 m d_{1}+f_{1} m_{s}+h_{1}\left(2 m+m_{s}\right) \\
2 \sqrt{2} m d_{1}+\sqrt{2} m f_{1}+h_{1}\left(2 \sqrt{2} m+\sqrt{2} m_{s}\right)\end{array}$ & $\begin{array}{cc}g_{\Xi_{c}^{\prime+} \Omega_{c}^{0}} & \sqrt{2} f_{1} m_{s}+d_{1}\left(\sqrt{2} m+\sqrt{2} m_{s}\right)+h_{1}\left(2 \sqrt{2} m+\sqrt{2} m_{s}\right) \\
g_{\Sigma_{c}^{+} \Xi_{c}^{\prime 0}} & m f_{1}+d_{1}\left(m+m_{s}\right)+h_{1}\left(2 m+m_{s}\right) \\
g_{\Sigma_{c}^{++} \Xi_{c}^{\prime+}} & \sqrt{2} m f_{1}+d_{1}\left(\sqrt{2} m+\sqrt{2} m_{s}\right)+h_{1}\left(2 \sqrt{2} m+\sqrt{2} m_{s}\right) \\
\end{array}$ \\
\hline $\begin{array}{l}g_{\Lambda_{c}^{+} \Sigma_{c}^{0}} \\
g_{\Xi_{c}^{+} \Xi_{c}^{\prime 0}}\end{array}$ & $\begin{array}{c}4 m d_{2}+2 m f_{2}+h_{2}\left(4 m+2 m_{s}\right) \\
2 \sqrt{2} m d_{2}+\sqrt{2} f_{2} m_{s}+h_{2}\left(2 \sqrt{2} m+\sqrt{2} m_{s}\right)\end{array}$ & $\begin{array}{c}\sqrt{2} m f_{2}+d_{2}\left(\sqrt{2} m+\sqrt{2} m_{s}\right)+h_{2}\left(2 \sqrt{2} m+\sqrt{2} m_{s}\right) \\
\sqrt{2} f_{5} m_{s}+d_{5}\left(\sqrt{2} m+\sqrt{2} m_{s}\right)+h_{5}\left(2 \sqrt{2} m+\sqrt{2} m_{s}\right) \\
-\sqrt{2} m f_{2}-d_{2}\left(\sqrt{2} m+\sqrt{2} m_{s}\right)-h_{2}\left(2 \sqrt{2} m+\sqrt{2} m_{s}\right) \\
-2 m f_{2}-d_{2}\left(2 m+2 m_{s}\right)-h_{2}\left(4 m+2 m_{s}\right) \\
\end{array}$ \\
\hline $\begin{array}{l}g_{\Xi_{c}^{* \prime}+\Xi_{c}^{* \prime 0}} \\
g_{\Sigma_{c}^{*+} \Sigma_{c}^{* 0}}\end{array}$ & $\begin{array}{c}2 m d_{5}+f_{5} m_{s}+h_{5}\left(2 m+m_{s}\right) \\
2 \sqrt{2} m d_{5}+\sqrt{2} m f_{5}+h_{5}\left(2 \sqrt{2} m+\sqrt{2} m_{s}\right)\end{array}$ & $\begin{array}{cc}g_{\Xi_{c}^{* \prime}+\Omega_{c}^{* 0}} & \sqrt{2} f_{5} m_{s}+d_{5}\left(\sqrt{2} m+\sqrt{2} m_{s}\right)+h_{5}\left(2 \sqrt{2} m+\sqrt{2} m_{s}\right) \\
g_{\Sigma_{c}^{*+} \Xi_{c}^{* \prime 0}} & m f_{5}+d_{5}\left(m+m_{s}\right)+h_{5}\left(2 m+m_{s}\right) \\
g_{\Sigma_{c}^{*++} \Xi_{c}^{* \prime+}} & \sqrt{2} m f_{5}+d_{5}\left(\sqrt{2} m+\sqrt{2} m_{s}\right)+h_{5}\left(2 \sqrt{2} m+\sqrt{2} m_{s}\right) \\
\end{array}$ \\
\hline $\begin{array}{l}g_{\Xi_{c}^{\prime+} \Xi_{c}^{* \prime 0}} \\
g_{\Sigma_{c}^{+} \Sigma_{c}^{* 0}} \\
g_{\Sigma_{c}^{++} \Sigma_{c}^{*+}}\end{array}$ & $\begin{array}{c}m d_{3}+h_{3}\left(m+\frac{m_{s}}{2}\right)+\frac{f_{3} m_{s}}{2} \\
\sqrt{2} m d_{3}+\frac{m f_{3}}{\sqrt{2}}+h_{3}\left(\sqrt{2} m+\frac{m_{s}}{\sqrt{2}}\right) \\
\sqrt{2} m d_{3}+\frac{m f_{3}}{\sqrt{2}}+h_{3}\left(\sqrt{2} m+\frac{m_{s}}{\sqrt{2}}\right)\end{array}$ & $\begin{array}{c}\frac{f_{3} m_{s}}{\sqrt{2}}+d_{3}\left(\frac{m}{\sqrt{2}}+\frac{m_{s}}{\sqrt{2}}\right)+h_{3}\left(\sqrt{2} m+\frac{m_{s}}{\sqrt{2}}\right) \\
\frac{1}{2} m f_{3}+d_{3}\left(\frac{m}{2}+\frac{m_{s}}{2}\right)+h_{3}\left(m+\frac{m_{s}}{2}\right) \\
\frac{m f_{3}}{\sqrt{2}}+d_{3}\left(\frac{m}{\sqrt{2}}+\frac{m_{s}}{\sqrt{2}}\right)+h_{3}\left(\sqrt{2} m+\frac{m_{s}}{\sqrt{2}}\right) \\
\frac{f_{3} m_{s}}{\sqrt{2}}+d_{3}\left(\frac{m}{\sqrt{2}}+\frac{m_{s}}{\sqrt{2}}\right)+h_{3}\left(\sqrt{2} m+\frac{m_{s}}{\sqrt{2}}\right) \\
\frac{m f_{3}}{2}+d_{3}\left(\frac{m}{2}+\frac{m_{s}}{2}\right)+h_{3}\left(m+\frac{m_{s}}{2}\right) \\
\frac{m f_{3}}{\sqrt{2}}+d_{3}\left(\frac{m}{\sqrt{2}}+\frac{m_{s}}{\sqrt{2}}\right)+h_{3}\left(\sqrt{2} m+\frac{m_{s}}{\sqrt{2}}\right) \\
\end{array}$ \\
\hline $\begin{array}{l}g_{\Lambda_{c}^{+} \Sigma_{c}^{* 0}} \\
g_{\Xi_{c}^{+} \Xi_{c}^{* \prime 0}} \\
\end{array}$ & $\begin{array}{c}2 m d_{4}+m f_{4}+h_{4}\left(2 m+m_{s}\right) \\
\sqrt{2} m d_{4}+\frac{f_{4} m_{s}}{\sqrt{2}}+h_{4}\left(\sqrt{2} m+\frac{m_{s}}{\sqrt{2}}\right) \\
\end{array}$ & $\begin{array}{r}\frac{m f_{4}}{\sqrt{2}}+d_{4}\left(\frac{m}{\sqrt{2}}+\frac{m_{s}}{\sqrt{2}}\right)+h_{4}\left(\sqrt{2} m+\frac{m_{s}}{\sqrt{2}}\right) \\
-\frac{m f_{4}}{\sqrt{2}}-d_{4}\left(\frac{m}{\sqrt{2}}+\frac{m_{s}}{\sqrt{2}}\right)-h_{4}\left(\sqrt{2} m+\frac{m_{s}}{\sqrt{2}}\right) \\
\end{array}$ \\
\hline
\end{tabular}

The renormalized matrix elements of the axial currents can be written as

$$
\begin{aligned}
\left\langle\mathcal{N}_{i}\left|A^{a, \mu}\left(g_{1,2}\right)\right| \mathcal{N}_{j}\right\rangle & =\bar{u}_{i} S^{\mu} u_{j}\left(g_{1,2(i j)}^{(0)}+g_{1,2(i j)}^{(2)}+g_{1,2(i j)}^{a}+g_{1,2(i j)}^{b}+g_{1,2(i j)}^{\mathrm{Re}}\right) \\
\left\langle\mathcal{T}_{i}^{\rho}\left|A^{a, \mu}\left(g_{5}\right)\right| \mathcal{T}_{j}^{\sigma}\right\rangle & =g_{\rho \sigma} \bar{u}_{i}^{\rho} S^{\mu} u_{j}^{\sigma}\left(g_{5(i j)}^{(0)}+g_{5(i j)}^{(2)}+g_{5(i j)}^{a}+g_{5(i j)}^{b}+g_{5(i j)}^{\mathrm{Re}}\right) \\
\left\langle\mathcal{N}_{i}\left|A^{a, \mu}\left(g_{3,4}\right)\right| \mathcal{T}_{j}^{\mu}\right\rangle & =\bar{u}_{i} u_{j}^{\mu}\left(g_{3,4(i j)}^{(0)}+g_{3,4(i j)}^{(2)}+g_{3,4(i j)}^{a}+g_{3,4(i j)}^{b}+g_{3,4(i j)}^{\mathrm{Re}}\right)
\end{aligned}
$$

$g_{(i j)}^{a, b}$ etc are the corrections at the one-loop level in Fig. 2. $g_{(i j)}^{\mathrm{Re}}$ etc. arise from the wave function renormalization.

\section{B. The axial currents correction on loop level}

At the one-loop level, there are four Feynman diagrams as shown in Fig. 2, where the filled circle represents the axial current vertex. Diagrams c and d arise from the chiral connection in Eq. (20).

FIG. 2: Vertex correction
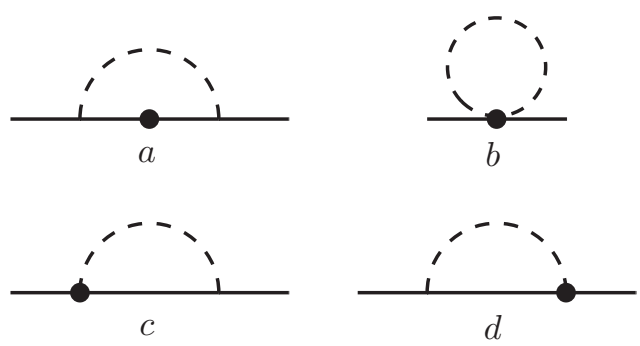

The vertex correction diagram (a) can be classified into three or four different types according to the Lorentz structure in the loop integrals, which are displayed in Figs. 3 [ 7 in Appendix VIA] For the vertex corrections to 
the axial charges $g_{1}$ and $g_{2}$, type I denotes the case that only the spin- $\frac{1}{2}$ baryons participate in the intermediate process. Type III contains only the spin- $\frac{3}{2}$ baryons as intermediate states. Type II contains both the spin- $\frac{1}{2}$ and spin- $\frac{3}{2}$ baryons. For the other axial charges, the classification of the vertex correction diagrams is similar.

With the contraction formulas between the spin projection operator $P_{(33) \mu \nu}^{\frac{3}{2}}$, Pauli-Lubanski vector $S^{\mu}$, and the metric $g_{\mu \nu}$ listed in Appendix VID we obtain the expressions of the axial currents from the vertex correction diagram (a).

$$
\begin{aligned}
& A_{i j}^{a, \mu}\left(g_{1}, g_{2}\right)_{\mathrm{I}}=g_{1,2(i j)}^{a} \bar{u}_{i} S^{\mu} u_{j}\left(a_{1,2, \mathrm{I}}+b_{1,2, \mathrm{I}} \epsilon\right) \frac{\Delta f}{\Delta \omega}, \quad a_{1,2, \mathrm{I}}+b_{1,2, \mathrm{I}} \epsilon=\frac{d-3}{4} \frac{-1}{d-1} \\
& A_{i j}^{a, \mu}\left(g_{1}, g_{2}\right)_{\mathrm{II}}=g_{1,2(i j)}^{a} \bar{u}_{i} S^{\mu} u_{j}\left(a_{1,2, \mathrm{II}}+b_{1,2, \mathrm{II}} \epsilon\right) \frac{\Delta f}{\Delta \omega}, \quad a_{1,2, \mathrm{II}}+b_{1,2, \mathrm{II}} \epsilon=\frac{2(d-2)}{d-1} \frac{-1}{d-1} \\
& A_{i j}^{a, \mu}\left(g_{1}, g_{2}\right)_{\mathrm{III}}=g_{1,2(i j)}^{a} \bar{u}_{i} S^{\mu} u_{j}\left(a_{1,2, \mathrm{III}}+b_{1,2, \mathrm{III}} \epsilon\right) \frac{\Delta f}{\Delta \omega}, \\
& a_{1,2, \mathrm{III}}+b_{1,2, \mathrm{III}} \epsilon=\frac{(d-3)(d-2)(d+1)}{(d-1)^{2}} \frac{-1}{d-1} \\
& A^{a, \mu}\left(g_{5}\right)_{\mathrm{I}}=g_{5(i j)}^{a} g_{\rho \sigma} \bar{u}_{j}^{\rho} S^{\mu} u_{j}^{\sigma}\left(a_{5, \mathrm{I}}+b_{5, \mathrm{I}} \epsilon\right) \frac{\Delta f}{\Delta \omega}, \quad a_{5, \mathrm{I}}+b_{5, \mathrm{I}} \epsilon=\frac{-1}{d-1} \\
& A^{a, \mu}\left(g_{5}\right)_{\mathrm{II}}=g_{5(i j)}^{a} g_{\rho \sigma} \bar{u}_{j}^{\rho} S^{\mu} u_{j}^{\sigma}\left(a_{5, \mathrm{II}}+b_{5, \mathrm{II}} \epsilon\right) \frac{\Delta f}{\Delta \omega}, \quad a_{5, \mathrm{II}}+b_{5, \mathrm{II}} \epsilon=\frac{-2}{d-1} \frac{-1}{d-1} \\
& A^{a, \mu}\left(g_{5}\right)_{\mathrm{III}}=g_{5(i j)}^{a} g_{\rho \sigma} \bar{u}_{j}^{\rho} S^{\mu} u_{j}^{\sigma}\left(a_{5, \mathrm{III}}+b_{5, \mathrm{III}} \epsilon\right) \frac{\Delta f}{\Delta \omega}, \quad a_{5, \mathrm{III}}+b_{5, \mathrm{III}} \epsilon=\frac{d^{3}-5 d^{2}+3 d-7}{4(d-1)^{2}} \frac{-1}{d-1} \\
& A^{a, \mu}\left(g_{3}, g_{4}\right)_{\mathrm{I}}=g_{3,4(i j)}^{a} \bar{u}_{i} u_{j}^{\mu}\left(a_{3,4, \mathrm{I}}+b_{3,4, \mathrm{I}} \epsilon\right) \frac{\Delta f}{\Delta \omega}, \quad a_{3,4, \mathrm{I}}+b_{3,4, \mathrm{I}} \epsilon=\left(-\frac{1}{2}\right) \frac{-1}{d-1} \\
& A^{a, \mu}\left(g_{3}, g_{4}\right)_{\mathrm{II}}=g_{3,4(i j)}^{a} \bar{u}_{i} u_{j}^{\mu}\left(a_{3,4, \mathrm{II}}+b_{3,4, \mathrm{II}} \epsilon\right) \frac{\Delta f}{\Delta \omega}, \quad a_{3,4, \mathrm{II}}+b_{3,4, \mathrm{II}} \epsilon=\frac{d-3}{d-1} \frac{-1}{d-1} \\
& A^{a, \mu}\left(g_{3}, g_{4}\right)_{\mathrm{III}}=g_{3,4(i j)}^{a} \bar{u}_{i} u_{j}^{\mu}\left(a_{3,4, \mathrm{III}}+b_{3,4, \mathrm{III}} \epsilon\right) \frac{\Delta f}{\Delta \omega}, \quad a_{3,4, \mathrm{III}}+b_{3,4, \mathrm{III} \epsilon}=\left(\frac{1-d}{4}+\frac{1}{d-1}\right) \frac{-1}{d-1} \\
& A^{a, \mu}\left(g_{3}, g_{4}\right)_{\mathrm{IV}}=g_{3,4(i j)}^{a} \bar{u}_{i} u_{j}^{\mu}\left(a_{3,4, \mathrm{IV}}+b_{3,4, \mathrm{IV}} \epsilon\right) \frac{\Delta f}{\Delta \omega}, \quad a_{3,4, \mathrm{IV}}+b_{3,4, \mathrm{IV}} \epsilon=\frac{(d-3)(d+1)}{2(d-1)^{2}} \frac{-1}{d-1}
\end{aligned}
$$

The parameters $a, b$ arise from the loop integration with the dimensional regularization scheme. The coefficients $g_{(i j)}^{a}$ are listed in Tables VIIIXII while the function $\frac{\Delta f}{\Delta \omega}$ is defined in Appendix VIC

$A^{b, \mu}$ is the correction from the vertex diagram (b):

$$
\begin{aligned}
A_{i j}^{b, \mu}\left(g_{1}, g_{2}\right) & =g_{1,2(i j)}^{b} \bar{u}_{i} S^{\mu} u_{j} I(m) \\
A^{b, \mu}\left(g_{5}\right) & =g_{5(i j)}^{b} g_{\rho \sigma} \bar{u}_{j}^{\rho} S^{\mu} u_{j}^{\sigma} I(m) \\
A^{b, \mu}\left(g_{3}, g_{4}\right) & =g_{3,4(i j)}^{b} \bar{u}_{i} u_{j}^{\mu} I(m)
\end{aligned}
$$

where the function $I(m)$ is defined in Appendix VIC. The corresponding coefficients $g_{(i j)}^{b}$ are collected in Table XIII. The loop corrections from diagrams (c) and (d) vanish in the heavy baryon limit $M_{B} \rightarrow \infty$. Their contributions are of higher order in the $\frac{1}{M_{D}}$ expansion. The analogous situation occurs in the nucleon octet case. Interested readers may refer to Refs. 13, 14].

The composite axial current operator also receives the correction from the wave function renormalization [35]. The renormalization factor can be derived from the self-energy function

$$
Z=\frac{1}{1-\Sigma^{\prime}} \approx 1+\Sigma^{\prime}, \quad \Sigma^{\prime}=\left.\frac{\partial \Sigma}{\partial(v \cdot k)}\right|_{v \cdot k, k^{2}=0}
$$

The matrix elements of the renormalized axial current between the initial and final states read

$$
\begin{aligned}
{ }^{r}\left\langle B_{i}\left|A^{a, \mu}\right| B_{j}\right\rangle^{r} & =\left\langle B_{i}\left|A^{a, \mu} \sqrt{Z_{i} Z_{j}}\right| B_{j}\right\rangle \\
& \left.=\left\langle B_{i}\left|A^{a, \mu}\right| B_{j}\right\rangle+\frac{1}{2}\left(\Sigma_{i}^{\prime}+\Sigma_{j}^{\prime}\right)\right]\left\langle B_{i}\left|A^{a, \mu}\right| B_{j}\right\rangle \\
& =\bar{u}_{i} S^{\mu} u_{j} g_{i j}^{(0)}\left(1+\lambda_{i j}\right)
\end{aligned}
$$


where $g_{i j}^{(0)}$ is the axial charge at the tree level. The coefficients $\lambda_{i j}$ are collected in Tables XIV XVIII Comparing with Eqs. (24)-(26), we have $g_{(i j)}^{\mathrm{Re}}=g_{i j}^{(0)} \lambda_{i j}$. The real part of $\Sigma^{\prime}$ reads

$$
\begin{aligned}
\Sigma_{\mathrm{Re}}^{\prime} & =\left.(A+B \epsilon)\left[\left(m^{2}-\omega^{2}\right) \frac{\partial J}{\partial \omega}-2 \omega J(m, \omega)\right]\right|_{\operatorname{Re}, \omega=\delta} \\
& =A\left[\left.\left(m^{2}-\omega^{2}\right) \frac{\partial J}{\partial \omega}\right|_{\operatorname{Re}}-\left.2 \omega J(m, \omega)\right|_{\operatorname{Re}}\right]-B \frac{1}{4 \pi^{2}}\left(m^{2}-3 \omega^{2}\right) .
\end{aligned}
$$

The function $J$ is defined in Appendix VIC.

\section{Numerical results of the chiral correction to the axial charge}

In principle, the axial charges of the heavy baryons can be extracted from the measurement of their semileptonic decays. However, there do not exist any experimental data now. The lack of the data renders the determination of the low energy constants $d, f, h$ etc very difficult.

TABLE VI: The chiral corrections to the axial charges.

\begin{tabular}{|c|c|c|c|c|c|}
\hline & $\begin{array}{l}\text { Loop-a [with the same spin } \\
\text { states in the loop only] }\end{array}$ & loop-a (full) & loop-b & $\begin{array}{c}\text { Wave function } \\
\text { renormalization effect }\end{array}$ & Fit values \\
\hline$g_{\Lambda_{c}^{+} \Sigma_{c}^{0}}$ & -0.06 & -0.38 & $\overline{0.22}$ & $\overline{0.31}$ & $1.46 \pm 0.44$ \\
\hline$g_{\Sigma_{c}^{+} \Sigma_{c}^{0}}$ & -0.04 & -0.27 & 0.32 & 0.32 & $1.46 \pm 0.44$ \\
\hline$g_{\Xi_{c}^{\prime+} \Omega_{c}^{0}}$ & -0.09 & -0.60 & 0.45 & 0.53 & $1.71 \pm 0.62^{\ddagger}$ \\
\hline$g_{\Sigma_{c}^{+} \Xi_{c}^{\prime 0}}$ & -0.03 & -0.24 & 0.32 & 0.27 & $1.32 \pm 0.31^{\ddagger}$ \\
\hline$g_{\Sigma_{c}^{++} \Xi_{c}^{\prime+}}$ & -0.04 & -0.34 & 0.45 & 0.38 & $1.46 \pm 0.44$ \\
\hline$g_{\Lambda_{c}^{+} \Sigma_{c}^{0}}$ & -0.04 & -0.29 & -0.27 & 0.51 & $-0.93 \pm 0.28$ \\
\hline$g_{\Xi_{c}^{+} \Xi_{c}^{\prime 0}}$ & -0.08 & -0.34 & -0.19 & 0.63 & $-0.93 \pm 0.28$ \\
\hline$g_{\Lambda_{c}^{+} \Xi_{c}^{\prime \prime}}$ & -0.05 & -0.34 & -0.28 & 0.33 & $-0.93 \pm 0.28$ \\
\hline$g_{\Xi_{c}^{+} \Omega_{c}^{0}}$ & -0.15 & -0.66 & -0.39 & 0.82 & $-1.21 \pm 0.51^{\ddagger}$ \\
\hline$g_{\Sigma_{c}^{+} \Xi_{c}^{0}}$ & 0.07 & 0.34 & 0.28 & -0.67 & $-0.61 \pm 0.28^{\ddagger}$ \\
\hline$g_{\Sigma_{c}^{++} \Xi_{c}^{+}}$ & 0.10 & 0.48 & 0.39 & -0.95 & $-0.62 \pm 0.39^{\ddagger}$ \\
\hline 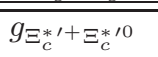 & 1.84 & 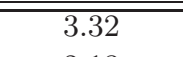 & -0.33 & -0.55 & $-2.19 \pm 0.66$ \\
\hline$g_{\Sigma_{c}^{*+} \Sigma_{c}^{* 0}}$ & 1.27 & 2.13 & -0.47 & -0.53 & $-2.19 \pm 0.66$ \\
\hline$g_{\Xi_{c}^{* \prime}+\Omega_{c}^{* 0}}$ & 2.37 & 5.09 & -0.67 & -1.00 & $-2.19 \pm 0.66$ \\
\hline$g_{\Sigma_{c}^{*+} \Xi_{c}^{* \prime 0}}$ & 0.82 & 1.84 & -0.48 & -0.46 & $-1.64 \pm 0.27^{\ddagger}$ \\
\hline$g_{\Sigma_{c}^{*++} \Xi_{c}^{* \prime+}}$ & 1.16 & 2.60 & -0.67 & -0.65 & $-1.71 \pm 0.38^{\ddagger}$ \\
\hline$g_{\Xi_{c}^{\prime+} \Xi_{c}^{* 0}}$ & & -0.29 & 0.10 & 0.15 & $1.26 \pm 0.38$ \\
\hline$g_{\Sigma_{c}^{+} \Sigma_{c}^{* 0}}$ & & -0.21 & 0.14 & 0.15 & $1.26 \pm 0.38$ \\
\hline$g_{\Sigma_{c}^{++} \Sigma_{c}^{*+}}$ & & -0.21 & 0.14 & 0.15 & $1.26 \pm 0.38^{\ddagger}$ \\
\hline$g_{\Xi_{c}^{\prime+}} \Omega_{c}^{* 0}$ & & -0.40 & 0.19 & 0.27 & $1.26 \pm 0.38$ \\
\hline$g_{\Sigma_{c}^{+} \Xi_{c}^{* \prime 0}}$ & & -0.14 & 0.14 & 0.13 & $1.01 \pm 0.16^{\ddagger}$ \\
\hline$g_{\Sigma_{c}^{++} \Xi_{c}^{* \prime+}}$ & & -0.20 & 0.19 & 0.18 & $1.06 \pm 0.22^{\ddagger}$ \\
\hline$g_{\Xi_{c}^{* \prime}+\Omega_{c}^{0}}$ & & -0.45 & 0.19 & 0.25 & $1.19 \pm 0.38^{\ddagger}$ \\
\hline$g_{\Sigma_{c}^{*+} \Xi_{c}^{\prime 0}}$ & & -0.21 & 0.14 & 0.12 & $0.93 \pm 0.16^{\ddagger}$ \\
\hline$g_{\Sigma_{c}^{*++}} \Xi_{c}^{\prime+}$ & & -0.30 & 0.19 & 0.17 & $0.95 \pm 0.22^{\ddagger}$ \\
\hline$g_{\Lambda_{c}^{+} \Sigma_{c}^{* 0}}$ & & 0.01 & 0.24 & -0.43 & $1.61 \pm 1.34$ \\
\hline$g_{\Xi_{c}^{+}} \Xi_{c}^{* \prime 0}$ & & -0.11 & 0.17 & -0.53 & $1.61 \pm 1.05$ \\
\hline$g_{\Lambda_{c}^{+} \Xi_{c}^{* \prime 0}}$ & & 0.07 & 0.24 & -0.26 & $1.14 \pm 1.19^{\ddagger}$ \\
\hline$g_{\Xi_{c}^{+} \Omega_{c}^{* 0}}$ & & -0.07 & 0.34 & -0.63 & $1.61 \pm 1.16$ \\
\hline
\end{tabular}

The marked entries are the predictions.

In Ref. [10], the authors calculated the pseudoscalar couplings of the heavy baryons. Within the framework of the chiral quark model, both the pseudoscalar couplings of the nucleons and heavy baryons can be expressed in terms of 
the pseudoscalar couplings of the constituent quarks. Since there exist plenty of nucleon nucleon scattering data, the pseudoscalar couplings of the nucleons can be determined very well experimentally. With the pion nucleon coupling as input, the authors first extracted the pseudoscalar couplings of the constituent quarks, and then determined the pseudoscalar couplings of the heavy baryons [10]. The axial charges are related to the coupling constants $g_{p B B}$

$$
g=\frac{2 F_{0}}{M_{a}+M_{b}} g_{p B B}
$$

From the values listed in Ref. [10], we have $g_{1}=1.46$ and $g_{2}=-0.93$. With the relationship among various $g$ 's in Sec. II] we get $g_{5}=-2.19, g_{3}=1.26, g_{4}=1.61$. In the following analysis, we regard the above values of the axial charge as the pseudoexperimental data and use them as input to extract various low energy constants. The values of LECs $d^{\prime}=B_{0} d, f^{\prime}=B_{0} f, h^{\prime}=B_{0} h\left(\mathrm{MeV}^{-1}\right)$ are

$$
\begin{gathered}
d_{1}^{\prime}=-0.9 \times 10^{-3}, f_{1}^{\prime}=2.6 \times 10^{-3}, h_{1}^{\prime}=0.7 \times 10^{-3} \\
d_{2}^{\prime}=1.2 \times 10^{-3}, f_{2}^{\prime}=-1.7 \times 10^{-3}, h_{2}^{\prime}=-1.3 \times 10^{-3} \\
d_{5}^{\prime}=2.4 \times 10^{-3}, f_{5}^{\prime}=-19.1 \times 10^{-3}, h_{5}^{\prime}=-11.5 \times 10^{-3} \\
d_{3}^{\prime}=0.3 \times 10^{-3}, f_{3}^{\prime}=4.5 \times 10^{-3}, h_{3}^{\prime}=4.1 \times 10^{-3} \\
d_{4}^{\prime}=-5.6 \times 10^{-3}, f_{4}^{\prime}=7.4 \times 10^{-3}, h_{4}^{\prime}=7.0 \times 10^{-3}
\end{gathered}
$$

In our numerical analysis we also need the values of the axial charges at $O(p) g_{1}^{(0)}=0.98, g_{2}^{(0)}=-0.60, g_{5}^{(0)}=$ $-1.47, g_{3}^{(0)}=0.85, g_{4}^{(0)}=1.04$. We collect the numerical results of the chiral corrections to the axial charges in Table VI We also list the separate contributions from the vertex correction and wave function renormalization. In the calculation of the self energy, we considered the isospin breaking effects because there exist plenty of data on the heavy baryon masses. However, in the case of the chiral correction to the axial charge, we have to work in the isospin symmetry limit because of the scarce data. Actually, to calculate the contributions of the wave function renormalization effects, we could not use the results in Sec. IV]directly because what we needed was the wave function renormalization factor, which is the derivative of the self-energy function $\left(\Sigma^{\prime}\right)$, not the self-energy function $(\Sigma)$ itself. The expression of $\Sigma^{\prime}$ can be seen in Eq. (32).

The second column in TableVI corresponds to the vertex corrections from diagram (a) where the intermediate and external heavy baryons have the same spin. The third column contains the contribution from all types of diagram (a). For the corrections from diagram (a), comparing the second and third columns, one notices that the values increase with the interactions between baryons with different spin. The contributions from diagram (b) and wave function renormalization are listed in the fourth and fifth columns. The last column is the fit value of the axial charge. From Table VI, we can see that the chiral expansion converges well. The axial charges with the notation $\ddagger$ in the last column are the predicted values. To show the sensitivity of the axial charges, we varied the input data by $10 \%$. The errors of all fit values are listed in the last column of Table VI

We have calculated the flavor $\mathrm{SU}(3)$ breaking chiral corrections to the axial charges of the heavy baryons in the exact isospin limit. We notice that the divergences from diagram (a) for the flavor structure (1+i2) can be absorbed by the counterterms completely. In contrast, the divergences from diagram (a) for the flavor structure (4+i5) cannot be absorbed by the counterterms completely with the explicit SU(3) breaking. Only in the exact SU(3) flavor symmetry limit, both divergences can be absorbed by the counterterms.

For example, let us consider the axial currents with the flavor $(1+\mathrm{i} 2) A_{\Xi_{c}^{+} \Xi_{c}^{\prime 0}}^{\mu}$ and $A_{\Xi_{c}^{\prime+} \Xi_{c}^{0}}^{\mu}$. The two processes occupy the same position in the weight diagram and their counterterms are the same due to the $\mathrm{SU}(3)$ symmetry at the tree level. The corrections to $A_{\Xi_{c}^{+} \Xi_{c}^{\prime 0}}^{\mu}$ from diagram (a) contain the $\pi^{0}$ loop and $K^{+}$loop. The corrections to $A_{\Xi_{c}^{\prime+} \Xi_{c}^{0}}$ contain the $\pi^{0}$ loop and $K^{0}$ loop. In the isospin symmetry limit even with explicit SU(3) symmetry breaking, $m_{K+}=m_{K^{0}}$. So the divergences are the same and can be canceled exactly.

On the other hand, for instance, the axial currents with the flavor $(4+\mathrm{i} 5) A_{\Lambda_{c}^{+} \Xi_{c}^{\prime 0}}^{\mu}$ and $A_{\Sigma_{c}^{0} \Xi_{c}^{0}}^{\mu}$ also have the same form of counterterms. The corrections to $A_{\Lambda_{c}^{+} \Xi_{c}^{\prime 0}}^{\mu}$ contain the $\pi^{0}$ loop and $\pi^{+}$loop. The corrections to $A_{\Sigma_{c}^{0} \Xi_{c}^{0}}^{\mu}$ contain the $\pi^{0}$ loop and $K^{+}$loop. In the isospin limit but with the explicit SU(3) flavor breaking, the divergence from the $\pi^{+}$and $K^{+}$loops cannot be canceled by the same counterterms. But in the SU(3) limit, both divergences are the same. That is to say, there are not enough counterterms to absorb the divergence in the $\mathrm{SU}(3)$ flavor breaking situation. However, 
the redundant divergences will be absorbed by the new LECs at higher order. We can drop the redundant infinities safely since the chiral symmetry ensures that the divergences can be absorbed into the higher order counterterms. The underlying reason is the asymmetry between the triplet and sextet representations in the weight diagram.

The axial currents between two sextet representations or two octet representations do not suffer from the above problems. The same situation occurs to the wave function renormalization. However, once again, the chiral symmetry ensures that the divergences can be absorbed into the higher order counterterms if the SU(3)-breaking terms are regarded as higher order.

\section{SUMMARY}

In short summary, we have calculated the one-loop chiral corrections to the masses and axial charges of the charmed antitriplet and sextet heavy baryon systems in the HBChPT framework.

After introducing the chiral Lagrangians, we have systematically calculated the baryon masses to the $O\left(p^{3}\right)$ due to the explicit SU(3) breaking. The mass splitting of the heavy baryons is related to up and down quark mass difference, and electric charge. Both strong interaction and QED effects are involved in our calculation. The resulting charmed baryon masses and decay widths are in good agreement with experimental data. The LECs are consistent with the values in Ref. [8, 9].

We have also calculated the chiral loop contributions from the vertex corrections and wave function renormalization to the axial charges of the heavy baryons in the isospin symmetry limit but with explicit SU(3) breaking. The convergence of the chiral expansion is quite good. In the future, the axial charges of the heavy baryons may be measured through their semileptonic and nonleptonic decays experimentally. The ongoing LHCb experiment and the future B factories will enrich the data of heavy baryons. Moreover, the axial charges play an important role in the study of the loosely bound molecular states composed of two heavy baryons.

The mass spectrum of the charmed and bottom baryons with different quark content and isospin has been computed with the Lattice NRQCD formalism [36]. The axial current of the bottom hadrons was explored by using partially quenched chiral perturbation theory in Lattice QCD [37]. Hopefully the expressions of the chiral loop corrections to the masses and axial charges of the heavy baryons will be useful in the chiral extrapolation of the lattice simulation data of these two quantities where the pion mass on the lattice is larger than its experimental value.

\section{ACKNOWLEDGMENTS}

N. Jiang is very grateful to Zhan-Wei Liu and Zhi-Feng Sun for very helpful discussions. This project is supported by the National Natural Science Foundation of China under Grant No. 11261130311. 
VI. APPENDIX

A. CATEGORIES OF THE VERTEX CORRECTION DIAGRAM (a).

FIG. 3: The flavor and Lorentz structures of diagram (a) for $g_{1}$.

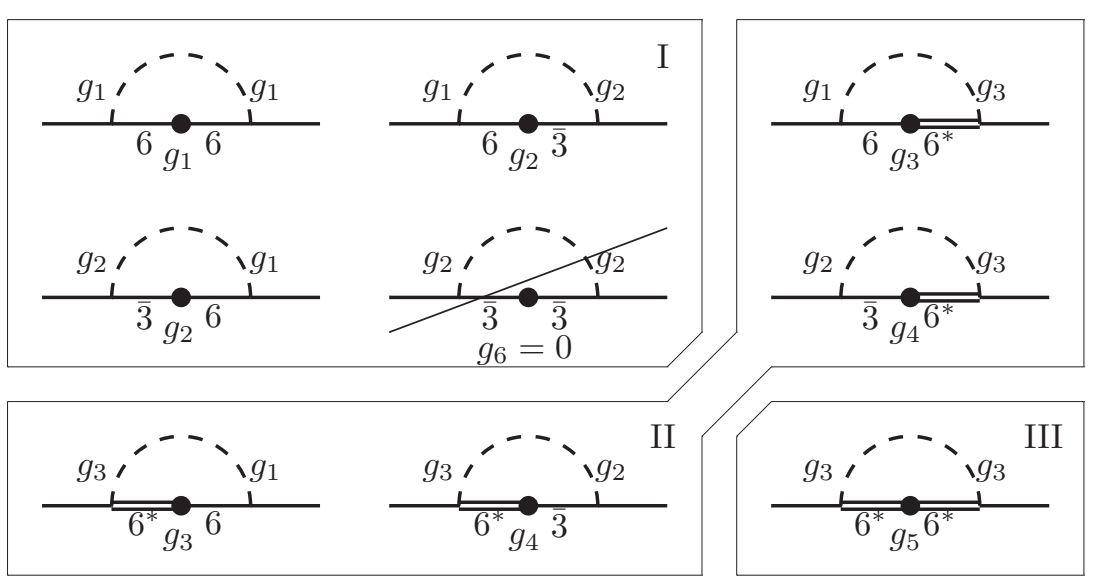

FIG. 4: The flavor and Lorentz structures of diagram (a) for $g_{2}$.
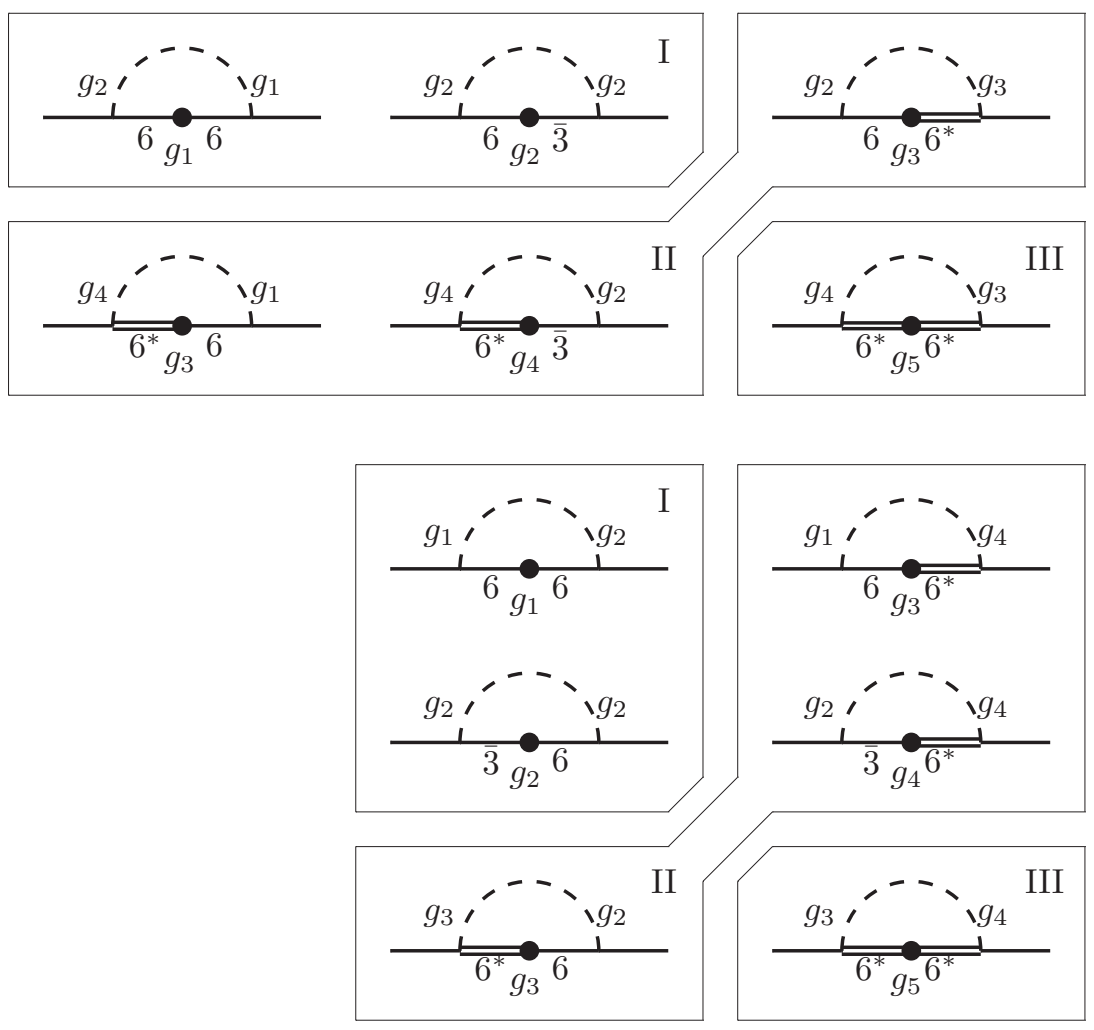
FIG. 5: The flavor and Lorentz structures of diagram (a) for $g_{5}$.

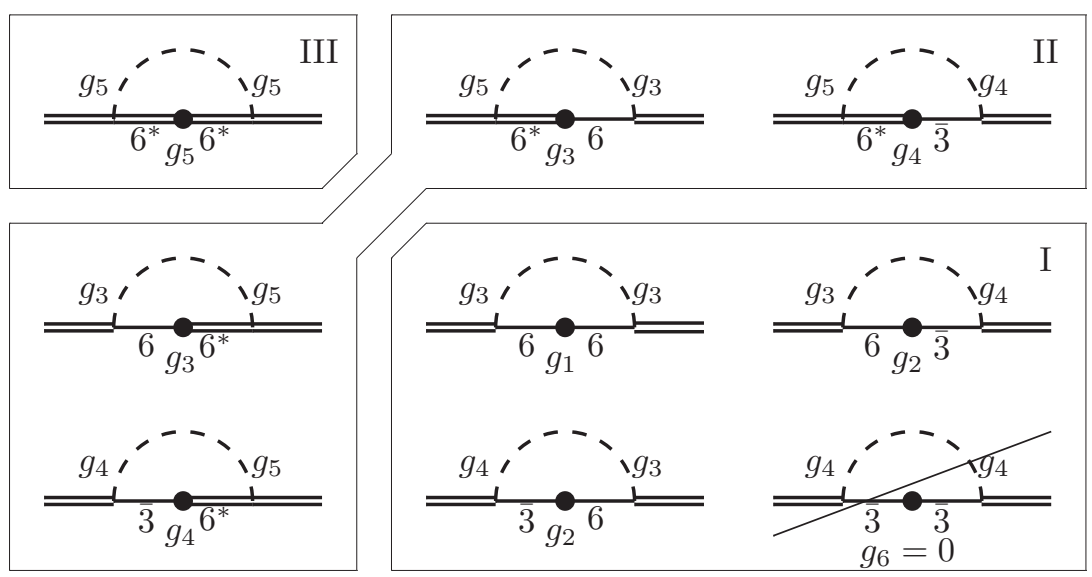

FIG. 6: The flavor and Lorentz structures of diagram (a) for $g_{3}$.
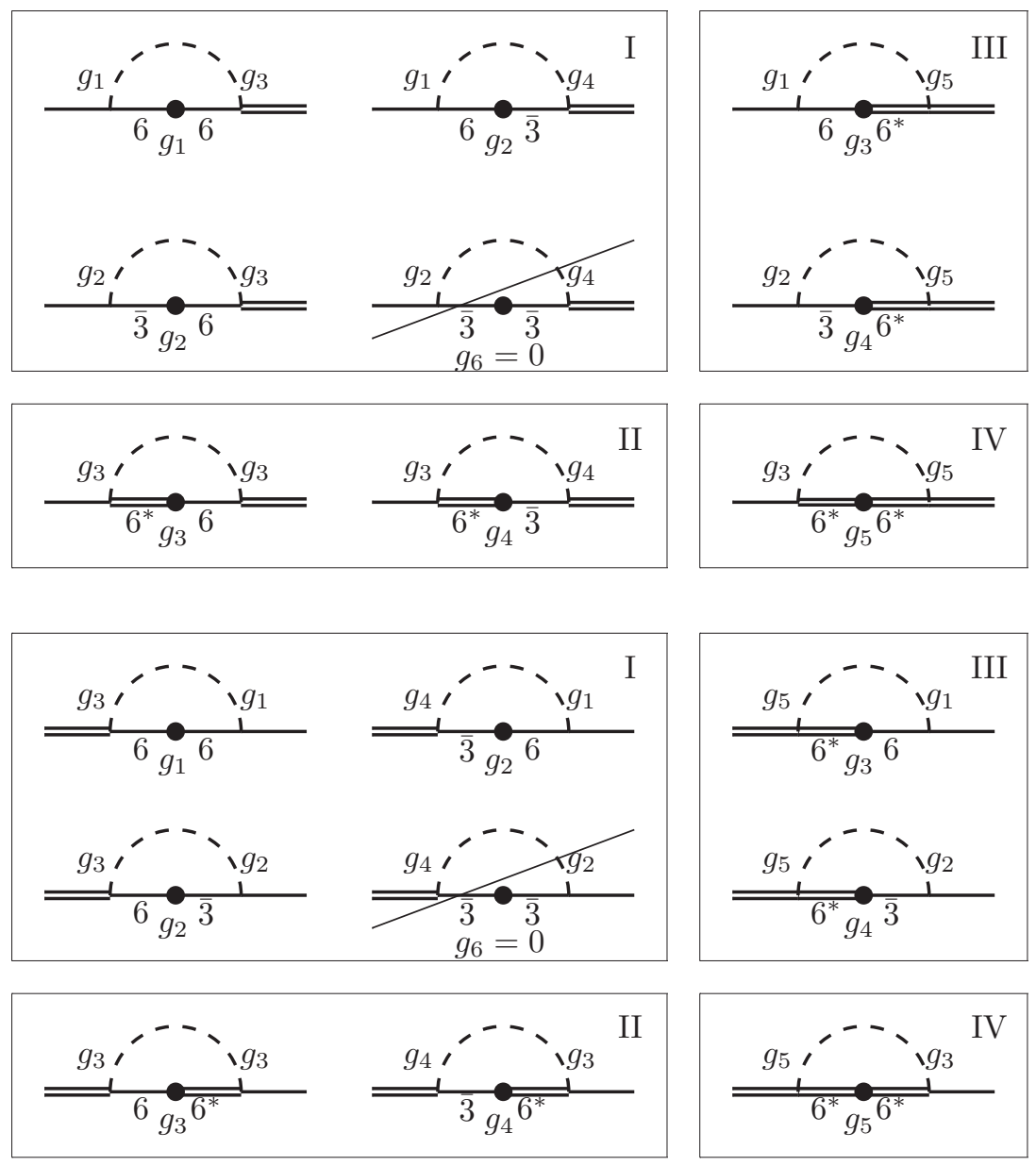
FIG. 7: The flavor and Lorentz structures of diagram (a) for $g_{4}$.

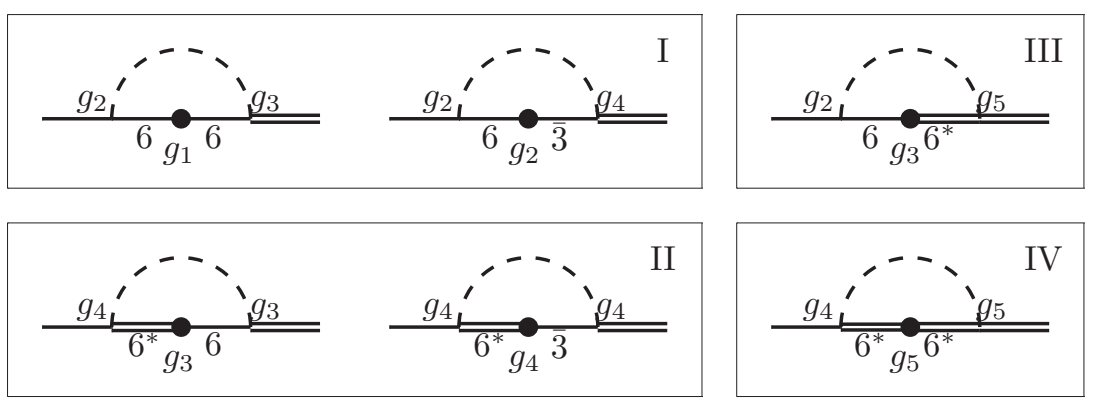

\section{B. TABLES}

TABLE VII: The coefficients $C$ in the self-energy function.

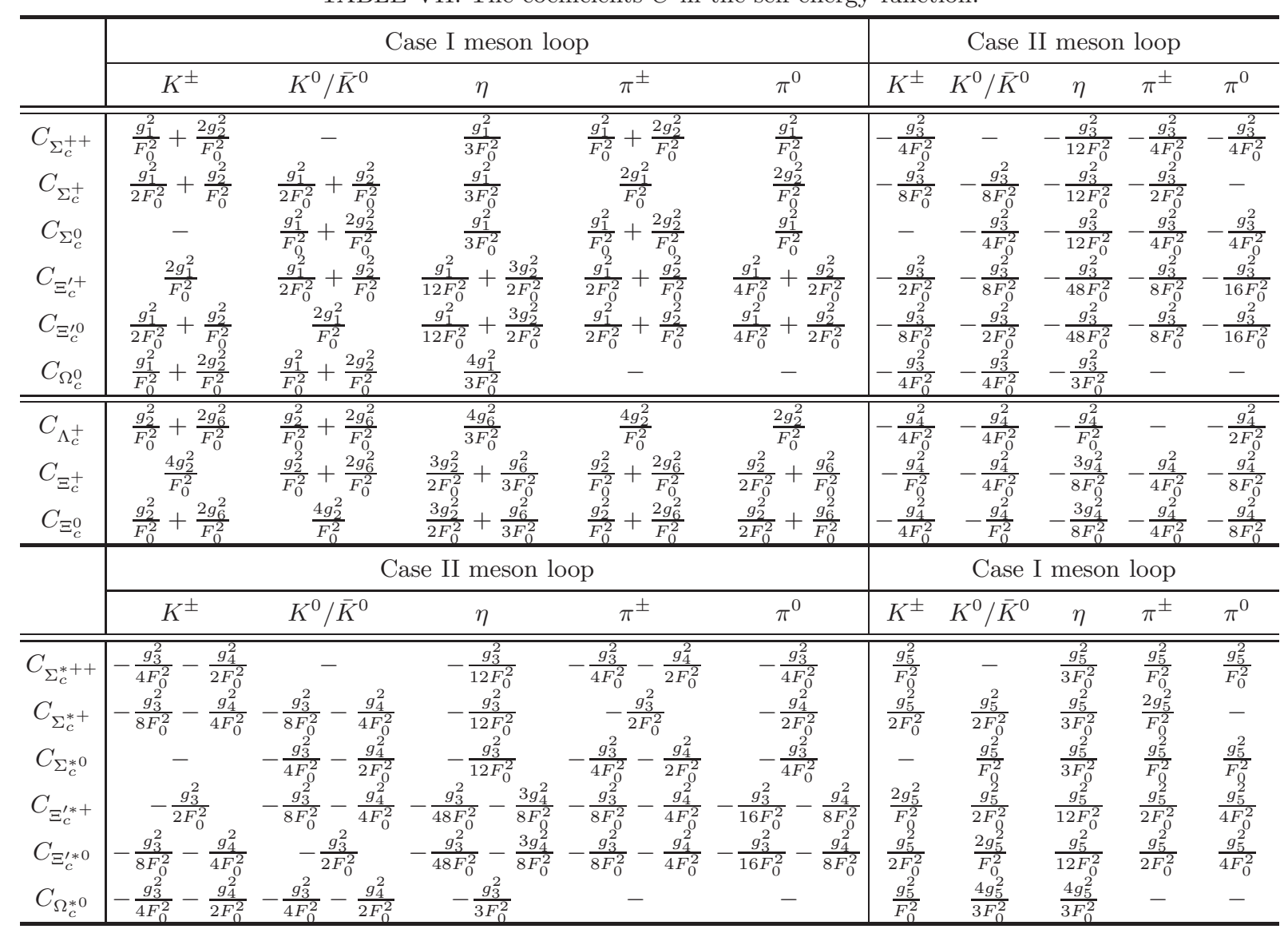


TABLE VIII: The coefficients $g_{1(i j)}^{a}$ of the axial current from diagram (a).

\begin{tabular}{|c|c|c|c|c|}
\hline & & Type I & Type II & Type III \\
\hline$g_{\Xi_{c}^{\prime+} \Xi_{c}^{\prime 0}}$ & $\begin{array}{l}K \text {-loop } \\
\eta \text {-loop } \\
\pi \text {-loop }\end{array}$ & $\begin{array}{c}\frac{2 g_{1}^{3}}{F_{0}^{2}}-\frac{4 g_{1} g_{2}^{2}}{F_{0}^{2}} \\
\frac{g_{1}^{3}}{12 F_{0}^{2}}-\frac{g_{1} g_{2}^{2}}{F_{0}^{2}} \\
-\frac{g_{1}^{3}}{4 F_{0}^{2}}-\frac{g_{2}^{2} g_{1}}{F_{0}^{2}}\end{array}$ & $\begin{array}{l}\frac{g_{2} g_{3} g_{4}}{F_{0}^{2}}-\frac{g_{1} g_{3}^{2}}{F_{0}^{2}} \\
\frac{g_{2} g_{3} g_{4}}{4 F_{0}^{2}}-\frac{g_{1} g_{3}^{2}}{24 F_{0}^{2}} \\
\frac{g_{1} g_{3}^{2}}{8 F_{0}^{2}}+\frac{g_{2} g_{4} g_{3}}{4 F_{0}^{2}}\end{array}$ & $\begin{array}{c}\frac{g_{3}^{2} g_{5}}{2 F_{0}^{2}} \\
\frac{g_{3}^{2} g_{5}}{48 F_{0}^{2}} \\
-\frac{g_{3}^{2} g_{5}}{16 F_{0}^{2}}\end{array}$ \\
\hline$g_{\Sigma_{c}^{+} \Sigma_{c}^{0}}$ & $\begin{array}{l}K \text {-loop } \\
\eta \text {-loop } \\
\pi \text {-loop }\end{array}$ & $\begin{array}{c}\frac{g_{1}^{3}}{\sqrt{2} F_{0}^{2}}-\frac{2 \sqrt{2} g_{1} g_{2}^{2}}{F_{0}^{2}} \\
\frac{\sqrt{2} g_{1}^{3}}{3 F_{0}^{2}} \\
\frac{\sqrt{2} g_{1}^{3}}{F_{0}^{2}}-\frac{4 \sqrt{2} g_{1} g_{2}^{2}}{F_{0}^{2}}\end{array}$ & $\begin{array}{c}\frac{g_{2} g_{3} g_{4}}{\sqrt{2} F_{0}^{2}}-\frac{g_{1} g_{3}^{2}}{2 \sqrt{2} F_{0}^{2}} \\
\quad-\frac{g_{1} g_{3}^{2}}{3 \sqrt{2} F_{0}^{2}} \\
\frac{\sqrt{2} g_{2} g_{3} g_{4}}{F_{0}^{2}}-\frac{g_{1} g_{3}^{2}}{\sqrt{2} F_{0}^{2}}\end{array}$ & $\begin{array}{l}\frac{g_{3}^{2} g_{5}}{4 \sqrt{2} F_{0}^{2}} \\
\frac{g_{3}^{2} g_{5}}{6 \sqrt{2} F_{0}^{2}} \\
\frac{g_{3}^{2} g_{5}}{2 \sqrt{2} F_{0}^{2}}\end{array}$ \\
\hline$g_{\Xi_{c}^{\prime+}} \Omega_{c}^{0}$ & $\begin{array}{l}K \text {-loop } \\
\eta \text {-loop } \\
\pi \text {-loop }\end{array}$ & $\begin{array}{c}\frac{3 g_{1}^{3}}{\sqrt{2} F_{0}^{2}}-\frac{4 \sqrt{2} g_{1} g_{2}^{2}}{F_{0}^{2}} \\
\frac{\sqrt{2} g_{1}^{3}}{3 F_{0}^{2}}-\frac{2 \sqrt{2} g_{1} g_{2}^{2}}{F_{0}^{2}} \\
-\end{array}$ & $\begin{array}{c}\frac{\sqrt{2} g_{2} g_{3} g_{4}}{F_{0}^{2}}-\frac{3 g_{1} g_{3}^{2}}{2 \sqrt{2} F_{0}^{2}} \\
\frac{g_{2} g_{3} g_{4}}{\sqrt{2} F_{0}^{2}}-\frac{g_{1} g_{3}^{2}}{3 \sqrt{2} F_{0}^{2}} \\
-\end{array}$ & $\begin{array}{l}\frac{3 g_{3}^{2} g_{5}}{4 \sqrt{2} F_{0}^{2}} \\
\frac{g_{3}^{2} g_{5}}{6 \sqrt{2} F_{0}^{2}} \\
-\end{array}$ \\
\hline$g_{\Sigma_{c}^{+} \Xi_{c}^{\prime 0}}$ & $\begin{array}{l}K \text {-loop } \\
\eta \text {-loop } \\
\pi \text {-loop }\end{array}$ & $\begin{array}{c}\frac{g_{1}^{3}}{F_{0}^{2}}-\frac{2 g_{1} g_{2}^{2}}{F_{0}^{2}} \\
-\frac{g_{1}^{3}}{6 F_{0}^{2}}-\frac{g_{2}^{2} g_{1}}{F_{0}^{2}} \\
\frac{g_{1}^{3}}{F_{0}^{2}}-\frac{3 g_{1} g_{2}^{2}}{F_{0}^{2}}\end{array}$ & $\begin{array}{l}\frac{g_{2} g_{3} g_{4}}{2 F_{0}^{2}}-\frac{g_{1} g_{3}^{2}}{2 F_{0}^{2}} \\
\frac{g_{1} g_{3}^{2}}{12 F_{0}^{2}}+\frac{g_{2} g_{4} g_{3}}{4 F_{0}^{2}} \\
\frac{3 g_{2} g_{3} g_{4}}{4 F_{0}^{2}}-\frac{g_{1} g_{3}^{2}}{2 F_{0}^{2}}\end{array}$ & $\begin{aligned} & \frac{g_{3}^{2} g_{5}}{4 F_{0}^{2}} \\
- & \frac{g_{3}^{2} g_{5}}{24 F_{0}^{2}} \\
& \frac{g_{3}^{2} g_{5}}{4 F_{0}^{2}}\end{aligned}$ \\
\hline$g_{\Sigma_{c}^{++}} \Xi_{c}^{\prime+}$ & $\begin{array}{l}K \text {-loop } \\
\eta \text {-loop } \\
\pi \text {-loop }\end{array}$ & $\begin{array}{c}\frac{\sqrt{2} g_{1}^{3}}{F_{0}^{2}}-\frac{2 \sqrt{2} g_{1} g_{2}^{2}}{F_{0}^{2}} \\
-\frac{g_{1}^{3}}{3 \sqrt{2} F_{0}^{2}}-\frac{\sqrt{2} g_{2}^{2} g_{1}}{F_{0}^{2}} \\
\frac{\sqrt{2} g_{1}^{3}}{F_{0}^{2}}-\frac{3 \sqrt{2} g_{1} g_{2}^{2}}{F_{0}^{2}}\end{array}$ & $\begin{array}{l}\frac{g_{2} g_{3} g_{4}}{\sqrt{2} F_{0}^{2}}-\frac{g_{1} g_{3}^{2}}{\sqrt{2} F_{0}^{2}} \\
\frac{g_{1} g_{3}^{2}}{6 \sqrt{2} F_{0}^{2}}+\frac{g_{2} g_{4} g_{3}}{2 \sqrt{2} F_{0}^{2}} \\
\frac{3 g_{2} g_{3} g_{4}}{2 \sqrt{2} F_{0}^{2}}-\frac{g_{1} g_{3}^{2}}{\sqrt{2} F_{0}^{2}}\end{array}$ & $\begin{array}{c}\frac{g_{3}^{2} g_{5}}{2 \sqrt{2} F_{0}^{2}} \\
-\frac{g_{3}^{2} g_{5}}{12 \sqrt{2} F_{0}^{2}} \\
\frac{g_{3}^{2} g_{5}}{2 \sqrt{2} F_{0}^{2}}\end{array}$ \\
\hline
\end{tabular}

TABLE IX: The coefficients $g_{2(i j)}^{a}$ of the axial current from diagram (a).

\begin{tabular}{|c|c|c|c|c|}
\hline & & Type I & Type II & Type III \\
\hline \multirow{3}{*}{$g_{\Lambda_{c}^{+} \Sigma_{c}^{0}}$} & $K$-loop & $\frac{2 g_{2}^{3}}{F_{0}^{2}}-\frac{g_{1}^{2} g_{2}}{F_{0}^{2}}$ & $\frac{g_{2} g_{3}^{2}}{4 F_{0}^{2}}+\frac{g_{1} g_{4} g_{3}}{4 F_{0}^{2}}-\frac{g_{2} g_{4}^{2}}{2 F_{0}^{2}}$ & $-\frac{g_{3} g_{4} g_{5}}{4 F_{0}^{2}}$ \\
\hline & $\eta$-loop & - & - & \\
\hline & $\pi$-loop & $\frac{4 g_{2}^{3}}{F_{0}^{2}}-\frac{4 g_{1}^{2} g_{2}}{F_{0}^{2}}$ & $\frac{g_{2} g_{3}^{2}}{F_{0}^{2}}+\frac{g_{1} g_{4} g_{3}}{F_{0}^{2}}-\frac{g_{2} g_{4}^{2}}{F_{0}^{2}}$ & $-\frac{g_{3} g_{4} g_{5}}{F_{0}^{2}}$ \\
\hline \multirow{3}{*}{$g_{\Xi_{c}^{+}} \Xi_{c}^{\prime 0}$} & $K$-loop & $\frac{2 \sqrt{2} g_{2}^{3}}{F_{0}^{2}}-\frac{2 \sqrt{2} g_{1}^{2} g_{2}}{F_{0}^{2}}$ & $\frac{g_{2} g_{3}^{2}}{\sqrt{2} F_{0}^{2}}+\frac{g_{1} g_{4} g_{3}}{\sqrt{2} F_{0}^{2}}-\frac{g_{2} g_{4}^{2}}{\sqrt{2} F_{0}^{2}}$ & $-\frac{g_{3} g_{4} g_{5}}{\sqrt{2} F_{0}^{2}}$ \\
\hline & $\eta$-loop & $\frac{3 g_{2}^{3}}{\sqrt{2} F_{0}^{2}}-\frac{g_{1}^{2} g_{2}}{2 \sqrt{2} F_{0}^{2}}$ & $\frac{g_{2} g_{3}^{2}}{8 \sqrt{2} F_{0}^{2}}+\frac{g_{1} g_{4} g_{3}}{8 \sqrt{2} F_{0}^{2}}-\frac{3 g_{2} g_{4}^{2}}{4 \sqrt{2} F_{0}^{2}}$ & $-\frac{g_{3} g_{4} g_{5}}{8 \sqrt{2} F_{0}^{2}}$ \\
\hline & $\pi$-loop & $-\frac{g_{2}^{3}}{\sqrt{2} F_{0}^{2}}-\frac{g_{1}^{2} g_{2}}{2 \sqrt{2} F_{0}^{2}}$ & $\frac{g_{2} g_{3}^{2}}{8 \sqrt{2} F_{0}^{2}}+\frac{g_{1} g_{4} g_{3}}{8 \sqrt{2} F_{0}^{2}}+\frac{g_{2} g_{4}^{2}}{4 \sqrt{2} F_{0}^{2}}$ & $-\frac{g_{3} g_{4} g_{5}}{8 \sqrt{2} F_{0}^{2}}$ \\
\hline \multirow{3}{*}{$g_{\Lambda_{c}^{+}} \Xi_{c}^{\prime 0}$} & $K$-loop & $-\frac{\sqrt{2} g_{1}^{2} g_{2}}{F_{0}^{2}}$ & $\frac{g_{2} g_{3}^{2}}{2 \sqrt{2} F_{0}^{2}}+\frac{g_{1} g_{4} g_{3}}{2 \sqrt{2} F_{0}^{2}}$ & $-\frac{g_{3} g_{4} g_{5}}{2 \sqrt{2} F_{0}^{2}}$ \\
\hline & $\eta$-loop & - & - & - \\
\hline & $\pi$-loop & $\frac{3 \sqrt{2} g_{2}^{3}}{F_{0}^{2}}-\frac{3 g_{1}^{2} g_{2}}{\sqrt{2} F_{0}^{2}}$ & $\frac{3 g_{2} g_{3}^{2}}{4 \sqrt{2} F_{0}^{2}}+\frac{3 g_{1} g_{4} g_{3}}{4 \sqrt{2} F_{0}^{2}}-\frac{3 g_{2} g_{4}^{2}}{2 \sqrt{2} F_{0}^{2}}$ & $-\frac{3 g_{3} g_{4} g_{5}}{4 \sqrt{2} F_{0}^{2}}$ \\
\hline \multirow{3}{*}{$g_{\Xi_{c}^{+} \Omega_{c}^{0}}$} & $K$-loop & $\frac{6 g_{2}^{3}}{F_{0}^{2}}-\frac{3 g_{1}^{2} g_{2}}{F_{0}^{2}}$ & $\frac{3 g_{2} g_{3}^{2}}{4 F_{0}^{2}}+\frac{3 g_{1} g_{4} g_{3}}{4 F_{0}^{2}}-\frac{3 g_{2} g_{4}^{2}}{2 F_{0}^{2}}$ & $-\frac{3 g_{3} g_{4} g_{5}}{4 F_{0}^{2}}$ \\
\hline & $\eta$-loop & $-\frac{2 g_{1}^{2} g_{2}}{F_{0}^{2}}$ & $\frac{g_{2} g_{3}^{2}}{2 F_{0}^{2}}+\frac{g_{1} g_{4} g_{3}}{2 F_{0}^{2}}$ & $-\frac{g_{3} g_{4} g_{5}}{2 F_{0}^{2}}$ \\
\hline & $\pi$-loop & - & - & - \\
\hline \multirow{3}{*}{$g_{\Sigma_{c}^{+}} \Xi_{c}^{+}$} & $K$-loop & $\frac{\sqrt{2} g_{1}^{2} g_{2}}{F_{0}^{2}}-\frac{2 \sqrt{2} g_{2}^{3}}{F_{0}^{2}}$ & $-\frac{g_{2} g_{3}^{2}}{2 \sqrt{2} F_{0}^{2}}-\frac{g_{1} g_{4} g_{3}}{2 \sqrt{2} F_{0}^{2}}+\frac{g_{2} g_{4}^{2}}{\sqrt{2} F_{0}^{2}}$ & $\frac{g_{3} g_{4} g_{5}}{2 \sqrt{2} F_{0}^{2}}$ \\
\hline & $\eta$-loop & $\frac{g_{1}^{2} g_{2}}{\sqrt{2} F_{0}^{2}}$ & $-\frac{g_{2} g_{3}^{2}}{4 \sqrt{2} F_{0}^{2}}-\frac{g_{1} g_{4} g_{3}}{4 \sqrt{2} F_{0}^{2}}$ & $\frac{g_{3} g_{4} g_{5}}{4 \sqrt{2} F_{0}^{2}}$ \\
\hline & $\pi$-loop & $\frac{\sqrt{2} g_{1}^{2} g_{2}}{F_{0}^{2}}-\frac{\sqrt{2} g_{2}^{3}}{F_{0}^{2}}$ & $-\frac{g_{2} g_{3}^{2}}{2 \sqrt{2} F_{0}^{2}}-\frac{g_{1} g_{4} g_{3}}{2 \sqrt{2} F_{0}^{2}}+\frac{g_{2} g_{4}^{2}}{2 \sqrt{2} F_{0}^{2}}$ & $\frac{g_{3} g_{4} g_{5}}{2 \sqrt{2} F_{0}^{2}}$ \\
\hline \multirow{3}{*}{$g_{\Sigma_{c}^{++}} \Xi_{c}^{+}$} & $K$-loop & $\frac{2 g_{1}^{2} g_{2}}{F_{0}^{2}}-\frac{4 g_{2}^{3}}{F_{0}^{2}}$ & $-\frac{g_{2} g_{3}^{2}}{2 F_{0}^{2}}-\frac{g_{1} g_{4} g_{3}}{2 F_{0}^{2}}+\frac{g_{2} g_{4}^{2}}{F_{0}^{2}}$ & $\frac{g_{3} g_{4} g_{5}}{2 F_{0}^{2}}$ \\
\hline & $\eta$-loop & $\frac{g_{1}^{2} g_{2}}{F_{0}^{2}}$ & $-\frac{g_{2} g_{3}^{2}}{4 F_{0}^{2}}-\frac{g_{1} g_{4} g_{3}}{4 F_{0}^{2}}$ & $\frac{g_{3} g_{4} g_{5}}{4 F_{0}^{2}}$ \\
\hline & $\pi$-loop & $\frac{2 g_{1}^{2} g_{2}}{F_{0}^{2}}-\frac{2 g_{2}^{3}}{F_{0}^{2}}$ & $-\frac{g_{2} g_{3}^{2}}{2 F_{0}^{2}}-\frac{g_{1} g_{4} g_{3}}{2 F_{0}^{2}}+\frac{g_{2} g_{4}^{2}}{2 F_{0}^{2}}$ & $\frac{g_{3} g_{4} g_{5}}{2 F_{0}^{2}}$ \\
\hline
\end{tabular}


TABLE X: The coefficients $g_{5(i j)}^{a}$ of the axial current from diagram (a).

\begin{tabular}{|c|c|c|c|c|}
\hline & & Type I & Type II & Type III \\
\hline$g_{\Xi_{c}^{* \prime}} \Xi_{c}^{* \prime 0}$ & $\begin{array}{l}K \text {-loop } \\
\eta \text {-loop } \\
\pi \text {-loop }\end{array}$ & $\begin{array}{c}\frac{g_{1} g_{3}^{2}}{2 F_{0}^{2}}-\frac{g_{2} g_{3} g_{4}}{F_{0}^{2}} \\
\frac{g_{1} g_{3}^{2}}{48 F_{0}^{2}}-\frac{g_{2} g_{3} g_{4}}{4 F_{0}^{2}} \\
-\frac{g_{1} g_{3}^{2}}{16 F_{0}^{2}}-\frac{g_{2} g_{4} g_{3}}{4 F_{0}^{2}} \\
\end{array}$ & $\begin{array}{l}\frac{g_{4}^{2} g_{5}}{F_{0}^{2}}-\frac{g_{3}^{2} g_{5}}{F_{0}^{2}} \\
\frac{g_{4}^{2} g_{5}}{4 F_{0}^{2}}-\frac{g_{3}^{2} g_{5}}{24 F_{0}^{2}} \\
\frac{g_{5} g_{3}^{2}}{8 F_{0}^{2}}+\frac{g_{4}^{2} g_{5}}{4 F_{0}^{2}}\end{array}$ & $\begin{array}{c}\frac{2 g_{5}^{3}}{F_{0}^{2}} \\
\frac{g_{5}^{3}}{12 F_{0}^{2}} \\
-\frac{g_{5}^{3}}{4 F_{0}^{2}}\end{array}$ \\
\hline$g_{\Sigma_{c}^{*+} \Sigma_{c}^{* 0}}$ & $\begin{array}{l}K \text {-loop } \\
\eta \text {-loop } \\
\pi \text {-loop }\end{array}$ & $\begin{array}{c}\frac{g_{1} g_{3}^{2}}{4 \sqrt{2} F_{0}^{2}}-\frac{g_{2} g_{3} g_{4}}{\sqrt{2} F_{0}^{2}} \\
\frac{g_{1} g_{3}^{2}}{6 \sqrt{2} F_{0}^{2}} \\
\frac{g_{1} g_{3}^{2}}{2 \sqrt{2} F_{0}^{2}}-\frac{\sqrt{2} g_{2} g_{3} g_{4}}{F_{0}^{2}}\end{array}$ & $\begin{array}{c}\frac{g_{4}^{2} g_{5}}{\sqrt{2} F_{0}^{2}}-\frac{g_{3}^{2} g_{5}}{2 \sqrt{2} F_{0}^{2}} \\
\quad-\frac{g_{3}^{2} g_{5}}{3 \sqrt{2} F_{0}^{2}} \\
\frac{\sqrt{2} g_{4}^{2} g_{5}}{F_{0}^{2}}-\frac{g_{3}^{2} g_{5}}{\sqrt{2} F_{0}^{2}}\end{array}$ & $\begin{array}{l}\frac{g_{5}^{3}}{\sqrt{2} F_{0}^{2}} \\
\frac{\sqrt{2} g_{5}^{3}}{3 F_{0}^{2}} \\
\frac{\sqrt{2} g_{5}^{3}}{F_{0}^{2}}\end{array}$ \\
\hline$g_{\Xi_{c}^{* \prime}}+\Omega_{c}^{* 0}$ & $\begin{array}{l}K \text {-loop } \\
\eta \text {-loop } \\
\pi \text {-loop }\end{array}$ & $\begin{array}{c}\frac{3 g_{1} g_{3}^{2}}{4 \sqrt{2} F_{0}^{2}}-\frac{\sqrt{2} g_{2} g_{3} g_{4}}{F_{0}^{2}} \\
\frac{g_{1} g_{3}^{2}}{6 \sqrt{2} F_{0}^{2}}-\frac{g_{2} g_{3} g_{4}}{\sqrt{2} F_{0}^{2}} \\
-\end{array}$ & $\begin{array}{c}\frac{\sqrt{2} g_{4}^{2} g_{5}}{F_{0}^{2}}-\frac{3 g_{3}^{2} g_{5}}{2 \sqrt{2} F_{0}^{2}} \\
\frac{g_{4}^{2} g_{5}}{\sqrt{2} F_{0}^{2}}-\frac{g_{3}^{2} g_{5}}{3 \sqrt{2} F_{0}^{2}} \\
-\end{array}$ & $\begin{array}{c}\frac{3 g_{5}^{3}}{\sqrt{2} F_{0}^{2}} \\
\frac{\sqrt{2} g_{5}^{3}}{3 F_{0}^{2}} \\
-\end{array}$ \\
\hline$g_{\Sigma_{c}^{*}+\Xi_{c}^{* \prime 0}}$ & $\begin{array}{c}K \text {-loop } \\
\eta \text {-loop } \\
\pi \text {-loop }\end{array}$ & $\begin{array}{c}\frac{g_{1} g_{3}^{2}}{4 F_{0}^{2}}-\frac{g_{2} g_{3} g_{4}}{2 F_{0}^{2}} \\
-\frac{g_{1} g_{3}^{2}}{24 F_{0}^{2}}-\frac{g_{2} g_{4} g_{3}}{4 F_{0}^{2}} \\
\frac{g_{1} g_{3}^{2}}{4 F_{0}^{2}}-\frac{3 g_{2} g_{3} g_{4}}{4 F_{0}^{2}}\end{array}$ & $\begin{array}{l}\frac{g_{4}^{2} g_{5}}{2 F_{0}^{2}}-\frac{g_{3}^{2} g_{5}}{2 F_{0}^{2}} \\
\frac{g_{5} g_{3}^{2}}{12 F_{0}^{2}}+\frac{g_{4}^{2} g_{5}}{4 F_{0}^{2}} \\
\frac{3 g_{4}^{2} g_{5}}{4 F_{0}^{2}}-\frac{g_{3}^{2} g_{5}}{2 F_{0}^{2}}\end{array}$ & $\begin{aligned} & \frac{g_{5}^{3}}{F_{0}^{2}} \\
&-\frac{g_{5}^{3}}{6 F_{0}^{2}} \\
& \frac{g_{5}^{3}}{F_{0}^{2}}\end{aligned}$ \\
\hline$g_{\Sigma_{c}^{*++} \Xi_{c}^{* \prime+}}$ & $\begin{array}{l}K \text {-loop } \\
\eta \text {-loop } \\
\pi \text {-loop }\end{array}$ & $\begin{array}{c}\frac{g_{1} g_{3}^{2}}{2 \sqrt{2} F_{0}^{2}}-\frac{g_{2} g_{3} g_{4}}{\sqrt{2} F_{0}^{2}} \\
-\frac{g_{1} g_{3}^{2}}{12 \sqrt{2} F_{0}^{2}}-\frac{g_{2} g_{4} g_{3}}{2 \sqrt{2} F_{0}^{2}} \\
\frac{g_{1} g_{3}^{2}}{2 \sqrt{2} F_{0}^{2}}-\frac{3 g_{2} g_{3} g_{4}}{2 \sqrt{2} F_{0}^{2}}\end{array}$ & $\begin{array}{l}\frac{g_{4}^{2} g_{5}}{\sqrt{2} F_{0}^{2}}-\frac{g_{3}^{2} g_{5}}{\sqrt{2} F_{0}^{2}} \\
\frac{g_{5} g_{3}^{2}}{6 \sqrt{2} F_{0}^{2}}+\frac{g_{4}^{2} g_{5}}{2 \sqrt{2} F_{0}^{2}} \\
\frac{3 g_{4}^{2} g_{5}}{2 \sqrt{2} F_{0}^{2}}-\frac{g_{3}^{2} g_{5}}{\sqrt{2} F_{0}^{2}}\end{array}$ & $\begin{aligned} & \frac{\sqrt{2} g_{5}^{3}}{F_{0}^{2}} \\
&-\frac{g_{5}^{3}}{3 \sqrt{2} F_{0}^{2}} \\
& \frac{\sqrt{2} g_{5}^{3}}{F_{0}^{2}}\end{aligned}$ \\
\hline
\end{tabular}


TABLE XI: The coefficients $g_{3(i j)}^{a}$ of the axial current from diagram (a).

\begin{tabular}{|c|c|c|c|c|c|}
\hline & & Type I & Type II & Type III & Type IV \\
\hline$g_{\Xi_{c}^{\prime+} \Xi_{c}^{* \prime 0}}$ & $\begin{array}{l}K \text {-loop } \\
\eta \text {-loop } \\
\pi \text {-loop }\end{array}$ & $\begin{array}{c}\frac{g_{3} g_{1}^{2}}{F_{0}^{2}}-\frac{g_{2} g_{4} g_{1}}{F_{0}^{2}}-\frac{g_{2}^{2} g_{3}}{F_{0}^{2}} \\
\frac{g_{3} g_{1}^{2}}{24 F_{0}^{2}}-\frac{g_{2} g_{4} g_{1}}{4 F_{0}^{2}}-\frac{g_{2}^{2} g_{3}}{4 F_{0}^{2}} \\
-\frac{g_{3} g_{1}^{2}}{8 F_{0}^{2}}-\frac{g_{2} g_{4} g_{1}}{4 F_{0}^{2}}-\frac{g_{2}^{2} g_{3}}{4 F_{0}^{2}} \\
\end{array}$ & $\begin{array}{l}\frac{g_{3} g_{4}^{2}}{4 F_{0}^{2}}-\frac{g_{3}^{3}}{4 F_{0}^{2}} \\
\frac{g_{3} g_{4}^{2}}{16 F_{0}^{2}}-\frac{g_{3}^{3}}{96 F_{0}^{2}} \\
\frac{g_{3}^{3}}{32 F_{0}^{2}}+\frac{g_{4}^{2} g_{3}}{16 F_{0}^{2}}\end{array}$ & $\begin{array}{l}\frac{g_{2} g_{4} g_{5}}{F_{0}^{2}}-\frac{g_{1} g_{3} g_{5}}{F_{0}^{2}} \\
\frac{g_{2} g_{4} g_{5}}{4 F_{0}^{2}}-\frac{g_{1} g_{3} g_{5}}{24 F_{0}^{2}} \\
\frac{g_{1} g_{3} g_{5}}{8 F_{0}^{2}}+\frac{g_{2} g_{4} g_{5}}{4 F_{0}^{2}}\end{array}$ & $\begin{array}{r}\frac{g_{3} g_{5}^{2}}{F_{0}^{2}} \\
\frac{g_{3} g_{5}^{2}}{24 F_{0}^{2}} \\
-\frac{g_{3} g_{5}^{2}}{8 F_{0}^{2}} \\
\end{array}$ \\
\hline$g_{\Sigma_{c}^{+} \Sigma_{c}^{* 0}}$ & $\begin{array}{l}K \text {-loop } \\
\eta \text {-loop } \\
\pi \text {-loop }\end{array}$ & $\begin{array}{c}\frac{g_{3} g_{1}^{2}}{2 \sqrt{2} F_{0}^{2}}-\frac{g_{2} g_{4} g_{1}}{\sqrt{2} F_{0}^{2}}-\frac{g_{2}^{2} g_{3}}{\sqrt{2} F_{0}^{2}} \\
\frac{g_{1}^{2} g_{3}}{3 \sqrt{2} F_{0}^{2}} \\
\frac{g_{3} g_{1}^{2}}{\sqrt{2} F_{0}^{2}}-\frac{\sqrt{2} g_{2} g_{4} g_{1}}{F_{0}^{2}}-\frac{\sqrt{2} g_{2}^{2} g_{3}}{F_{0}^{2}}\end{array}$ & $\begin{array}{l}\frac{g_{3} g_{4}^{2}}{4 \sqrt{2} F_{0}^{2}}-\frac{g_{3}^{3}}{8 \sqrt{2} F_{0}^{2}} \\
\quad-\frac{g_{3}^{3}}{12 \sqrt{2} F_{0}^{2}} \\
\frac{g_{3} g_{4}^{2}}{2 \sqrt{2} F_{0}^{2}}-\frac{g_{3}^{3}}{4 \sqrt{2} F_{0}^{2}} \\
\end{array}$ & $\begin{array}{c}\frac{g_{2} g_{4} g_{5}}{\sqrt{2} F_{0}^{2}}-\frac{g_{1} g_{3} g_{5}}{2 \sqrt{2} F_{0}^{2}} \\
\quad-\frac{g_{1} g_{3} g_{5}}{3 \sqrt{2} F_{0}^{2}} \\
\frac{\sqrt{2} g_{2} g_{4} g_{5}}{F_{0}^{2}}-\frac{g_{1} g_{3} g_{5}}{\sqrt{2} F_{0}^{2}}\end{array}$ & $\begin{array}{l}\frac{g_{3} g_{5}^{2}}{2 \sqrt{2} F_{0}^{2}} \\
\frac{g_{3} g_{5}^{2}}{3 \sqrt{2} F_{0}^{2}} \\
\frac{g_{3} g_{5}^{2}}{\sqrt{2} F_{0}^{2}} \\
\end{array}$ \\
\hline$g_{\Sigma_{c}^{+-}}$ & $\begin{array}{l}K \text {-loop } \\
\eta \text {-loop } \\
\pi \text {-loop }\end{array}$ & $\begin{array}{c}\frac{g_{3} g_{1}^{2}}{2 \sqrt{2} F_{0}^{2}}-\frac{g_{2} g_{4} g_{1}}{\sqrt{2} F_{0}^{2}}-\frac{g_{2}^{2} g_{3}}{\sqrt{2} F_{0}^{2}} \\
\frac{g_{1}^{2} g_{3}}{3 \sqrt{2} F_{0}^{2}} \\
\frac{g_{3} g_{1}^{2}}{\sqrt{2} F_{0}^{2}}-\frac{\sqrt{2} g_{2} g_{4} g_{1}}{F_{0}^{2}}-\frac{\sqrt{2} g_{2}^{2} g_{3}}{F_{0}^{2}}\end{array}$ & $\begin{array}{c}\frac{g_{3} g_{4}^{2}}{4 \sqrt{2} F_{0}^{2}}-\frac{g_{3}^{3}}{8 \sqrt{2} F_{0}^{2}} \\
\quad-\frac{g_{3}^{3}}{12 \sqrt{2} F_{0}^{2}} \\
\frac{g_{3} g_{4}^{2}}{2 \sqrt{2} F_{0}^{2}}-\frac{g_{3}^{3}}{4 \sqrt{2} F_{0}^{2}}\end{array}$ & $\begin{array}{c}\frac{g_{2} g_{4} g_{5}}{\sqrt{2} F_{0}^{2}}-\frac{g_{1} g_{3} g_{5}}{2 \sqrt{2} F_{0}^{2}} \\
\quad-\frac{g_{1} g_{3} g_{5}}{3 \sqrt{2} F_{0}^{2}} \\
\frac{\sqrt{2} g_{2} g_{4} g_{5}}{F_{0}^{2}}-\frac{g_{1} g_{3} g_{5}}{\sqrt{2} F_{0}^{2}}\end{array}$ & $\begin{array}{l}\frac{g_{3} g_{5}^{2}}{2 \sqrt{2} F_{0}^{2}} \\
\frac{g_{3} g_{5}^{2}}{3 \sqrt{2} F_{0}^{2}} \\
\frac{g_{3} g_{5}^{2}}{\sqrt{2} F_{0}^{2}}\end{array}$ \\
\hline$g_{\Xi_{c}}+\Omega_{c}^{* 0}$ & $\begin{array}{l}K \text {-loop } \\
\eta \text {-loop } \\
\pi \text {-loop }\end{array}$ & $\begin{array}{c}\frac{3 g_{3} g_{1}^{2}}{2 \sqrt{2} F_{0}^{2}}-\frac{3 g_{2} g_{4} g_{1}}{\sqrt{2} F_{0}^{2}}-\frac{g_{2}^{2} g_{3}}{\sqrt{2} F_{0}^{2}} \\
\frac{g_{1}^{2} g_{3}}{3 \sqrt{2} F_{0}^{2}}-\frac{\sqrt{2} g_{2}^{2} g_{3}}{F_{0}^{2}} \\
-\end{array}$ & $\begin{array}{c}\frac{3 g_{3} g_{4}^{2}}{4 \sqrt{2} F_{0}^{2}}-\frac{3 g_{3}^{3}}{8 \sqrt{2} F_{0}^{2}} \\
-\frac{g_{3}^{3}}{12 \sqrt{2} F_{0}^{2}} \\
-\end{array}$ & $\begin{array}{c}\frac{g_{2} g_{4} g_{5}}{\sqrt{2} F_{0}^{2}}-\frac{3 g_{1} g_{3} g_{5}}{2 \sqrt{2} F_{0}^{2}} \\
\frac{\sqrt{2} g_{2} g_{4} g_{5}}{F_{0}^{2}}-\frac{g_{1} g_{3} g_{5}}{3 \sqrt{2} F_{0}^{2}} \\
-\end{array}$ & $\begin{array}{l}\frac{3 g_{3} g_{5}^{2}}{2 \sqrt{2} F_{0}^{2}} \\
\frac{g_{3} g_{5}^{2}}{3 \sqrt{2} F_{0}^{2}} \\
-\end{array}$ \\
\hline$g_{\Sigma_{c}^{+} \Xi_{c}^{* \prime+}}$ & $\begin{array}{l}K \text {-loop } \\
\eta \text {-loop } \\
\pi \text {-loop }\end{array}$ & $\begin{array}{c}\frac{g_{1}^{2} g_{3}}{2 F_{0}^{2}}-\frac{g_{2}^{2} g_{3}}{F_{0}^{2}} \\
-\frac{g_{3} g_{1}^{2}}{12 F_{0}^{2}}-\frac{g_{2} g_{4} g_{1}}{2 F_{0}^{2}} \\
\frac{g_{3} g_{1}^{2}}{2 F_{0}^{2}}-\frac{g_{2} g_{4} g_{1}}{F_{0}^{2}}-\frac{g_{2}^{2} g_{3}}{2 F_{0}^{2}}\end{array}$ & $\begin{array}{c}-\frac{g_{3}^{3}}{8 F_{0}^{2}} \\
\frac{g_{3}^{3}}{48 F_{0}^{2}}+\frac{g_{4}^{2} g_{3}}{8 F_{0}^{2}} \\
\frac{g_{3} g_{4}^{2}}{4 F_{0}^{2}}-\frac{g_{3}^{3}}{8 F_{0}^{2}}\end{array}$ & $\begin{array}{l}\frac{g_{2} g_{4} g_{5}}{F_{0}^{2}}-\frac{g_{1} g_{3} g_{5}}{2 F_{0}^{2}} \\
\quad \frac{g_{1} g_{3} g_{5}}{12 F_{0}^{2}} \\
\frac{g_{2} g_{4} g_{5}}{2 F_{0}^{2}}-\frac{g_{1} g_{3} g_{5}}{2 F_{0}^{2}}\end{array}$ & $\begin{aligned} & \frac{g_{3} g_{5}^{2}}{2 F_{0}^{2}} \\
&- \frac{g_{3} g_{5}^{2}}{12 F_{0}^{2}} \\
& \frac{g_{3} g_{5}^{2}}{2 F_{0}^{2}} \\
&\end{aligned}$ \\
\hline$E_{c}^{* \prime+}$ & $\begin{array}{l}K \text {-loop } \\
\eta \text {-loop } \\
\pi \text {-loop }\end{array}$ & $\begin{array}{c}\frac{g_{1}^{2} g_{3}}{\sqrt{2} F_{0}^{2}}-\frac{\sqrt{2} g_{2}^{2} g_{3}}{F_{0}^{2}} \\
-\frac{g_{3} g_{1}^{2}}{6 \sqrt{2} F_{0}^{2}}-\frac{g_{2} g_{4} g_{1}}{\sqrt{2} F_{0}^{2}} \\
\frac{g_{3} g_{1}^{2}}{\sqrt{2} F_{0}^{2}}-\frac{\sqrt{2} g_{2} g_{4} g_{1}}{F_{0}^{2}}-\frac{g_{2}^{2} g_{3}}{\sqrt{2} F_{0}^{2}}\end{array}$ & $\begin{array}{c}-\frac{g_{3}^{3}}{4 \sqrt{2} F_{0}^{2}} \\
\frac{g_{3}^{3}}{24 \sqrt{2} F_{0}^{2}}+\frac{g_{4}^{2} g_{3}}{4 \sqrt{2} F_{0}^{2}} \\
\frac{g_{3} g_{4}^{2}}{2 \sqrt{2} F_{0}^{2}}-\frac{g_{3}^{3}}{4 \sqrt{2} F_{0}^{2}}\end{array}$ & $\begin{array}{c}\frac{\sqrt{2} g_{2} g_{4} g_{5}}{F_{0}^{2}}-\frac{g_{1} g_{3} g_{5}}{\sqrt{2} F_{0}^{2}} \\
\frac{g_{1} g_{3} g_{5}}{6 \sqrt{2} F_{0}^{2}} \\
\frac{g_{2} g_{4} g_{5}}{\sqrt{2} F_{0}^{2}}-\frac{g_{1} g_{3} g_{5}}{\sqrt{2} F_{0}^{2}}\end{array}$ & $\begin{aligned} & \frac{g_{3} g_{5}^{2}}{\sqrt{2} F_{0}^{2}} \\
&- \frac{g_{3} g_{5}^{2}}{6 \sqrt{2} F_{0}^{2}} \\
& \frac{g_{3} g_{5}^{2}}{\sqrt{2} F_{0}^{2}} \\
&\end{aligned}$ \\
\hline$g_{\Xi_{c}^{* \prime}}+\Omega_{c}^{0}$ & $\begin{array}{l}K \text {-loop } \\
\eta \text {-loop } \\
\pi \text {-loop }\end{array}$ & $\begin{array}{c}\frac{3 g_{3} g_{1}^{2}}{2 \sqrt{2} F_{0}^{2}}-\frac{g_{2} g_{4} g_{1}}{\sqrt{2} F_{0}^{2}}-\frac{3 g_{2}^{2} g_{3}}{\sqrt{2} F_{0}^{2}} \\
\frac{g_{1}^{2} g_{3}}{3 \sqrt{2} F_{0}^{2}}-\frac{\sqrt{2} g_{1} g_{2} g_{4}}{F_{0}^{2}} \\
-\end{array}$ & $\begin{array}{c}\frac{g_{3} g_{4}^{2}}{4 \sqrt{2} F_{0}^{2}}-\frac{3 g_{3}^{3}}{8 \sqrt{2} F_{0}^{2}} \\
\frac{g_{3} g_{4}^{2}}{2 \sqrt{2} F_{0}^{2}}-\frac{g_{3}^{3}}{12 \sqrt{2} F_{0}^{2}} \\
-\end{array}$ & $\begin{array}{c}\frac{3 g_{2} g_{4} g_{5}}{\sqrt{2} F_{0}^{2}}-\frac{3 g_{1} g_{3} g_{5}}{2 \sqrt{2} F_{0}^{2}} \\
-\frac{g_{1} g_{3} g_{5}}{3 \sqrt{2} F_{0}^{2}} \\
-\end{array}$ & $\begin{array}{l}\frac{3 g_{3} g_{5}^{2}}{2 \sqrt{2} F_{0}^{2}} \\
\frac{g_{3} g_{5}^{2}}{3 \sqrt{2} F_{0}^{2}} \\
-\end{array}$ \\
\hline$g_{\Sigma_{c}^{*+}} \Xi_{c}^{\prime+}$ & $\begin{array}{l}K \text {-loop } \\
\eta \text {-loop } \\
\pi \text {-loop }\end{array}$ & $\begin{array}{c}\frac{g_{1}^{2} g_{3}}{2 F_{0}^{2}}-\frac{g_{1} g_{2} g_{4}}{F_{0}^{2}} \\
-\frac{g_{3} g_{1}^{2}}{12 F_{0}^{2}}-\frac{g_{2}^{2} g_{3}}{2 F_{0}^{2}} \\
\frac{g_{3} g_{1}^{2}}{2 F_{0}^{2}}-\frac{g_{2} g_{4} g_{1}}{2 F_{0}^{2}}-\frac{g_{2}^{2} g_{3}}{F_{0}^{2}} \\
\end{array}$ & $\begin{array}{l}\frac{g_{3} g_{4}^{2}}{4 F_{0}^{2}}-\frac{g_{3}^{3}}{8 F_{0}^{2}} \\
\quad \frac{g_{3}^{3}}{48 F_{0}^{2}} \\
\frac{g_{3} g_{4}^{2}}{8 F_{0}^{2}}-\frac{g_{3}^{3}}{8 F_{0}^{2}}\end{array}$ & $\begin{array}{c}-\frac{g_{1} g_{3} g_{5}}{2 F_{0}^{2}} \\
\frac{g_{1} g_{3} g_{5}}{12 F_{0}^{2}}+\frac{g_{2} g_{4} g_{5}}{2 F_{0}^{2}} \\
\frac{g_{2} g_{4} g_{5}}{F_{0}^{2}}-\frac{g_{1} g_{3} g_{5}}{2 F_{0}^{2}}\end{array}$ & $\begin{aligned} & \frac{g_{3} g_{5}^{2}}{2 F_{0}^{2}} \\
&- \frac{g_{3} g_{5}^{2}}{12 F_{0}^{2}} \\
& \frac{g_{3} g_{5}^{2}}{2 F_{0}^{2}} \\
&\end{aligned}$ \\
\hline$g_{\Sigma_{c}^{*++} \Xi_{c}^{\prime+}}$ & $\begin{array}{l}K \text {-loop } \\
\eta \text {-loop } \\
\pi \text {-loop }\end{array}$ & $\begin{array}{c}\frac{g_{1}^{2} g_{3}}{\sqrt{2} F_{0}^{2}}-\frac{\sqrt{2} g_{1} g_{2} g_{4}}{F_{0}^{2}} \\
-\frac{g_{3} g_{1}^{2}}{6 \sqrt{2} F_{0}^{2}}-\frac{g_{2}^{2} g_{3}}{\sqrt{2} F_{0}^{2}} \\
\frac{g_{3} g_{1}^{2}}{\sqrt{2} F_{0}^{2}}-\frac{g_{2} g_{4} g_{1}}{\sqrt{2} F_{0}^{2}}-\frac{\sqrt{2} g_{2}^{2} g_{3}}{F_{0}^{2}}\end{array}$ & $\begin{array}{c}\frac{g_{3} g_{4}^{2}}{2 \sqrt{2} F_{0}^{2}}-\frac{g_{3}^{3}}{4 \sqrt{2} F_{0}^{2}} \\
\frac{g_{3}^{3}}{24 \sqrt{2} F_{0}^{2}} \\
\frac{g_{3} g_{4}^{2}}{4 \sqrt{2} F_{0}^{2}}-\frac{g_{3}^{3}}{4 \sqrt{2} F_{0}^{2}}\end{array}$ & $\begin{array}{c}-\frac{g_{1} g_{3} g_{5}}{\sqrt{2} F_{0}^{2}} \\
\frac{g_{1} g_{3} g_{5}}{6 \sqrt{2} F_{0}^{2}}+\frac{g_{2} g_{4} g_{5}}{\sqrt{2} F_{0}^{2}} \\
\frac{\sqrt{2} g_{2} g_{4} g_{5}}{F_{0}^{2}}-\frac{g_{1} g_{3} g_{5}}{\sqrt{2} F_{0}^{2}}\end{array}$ & $\begin{array}{l}\frac{g_{3} g_{5}^{2}}{\sqrt{2} F_{0}^{2}} \\
-\frac{g_{3} g_{5}^{2}}{6 \sqrt{2} F_{0}^{2}} \\
\frac{g_{3} g_{5}^{2}}{\sqrt{2} F_{0}^{2}} \\
\end{array}$ \\
\hline
\end{tabular}


TABLE XII: The coefficients $g_{4(i j)}^{a}$ of the axial current from diagram (a).

\begin{tabular}{|c|c|c|c|c|c|}
\hline & & Type I & Type II & Type III & Type IV \\
\hline \multirow{3}{*}{$g_{\Lambda_{c}^{+} \Sigma_{c}^{* 0}}$} & $K$-loop & $\frac{g_{2}^{2} g_{4}}{F_{0}^{2}}-\frac{g_{1} g_{2} g_{3}}{2 F_{0}^{2}}$ & $\frac{g_{3}^{2} g_{4}}{8 F_{0}^{2}}-\frac{g_{4}^{3}}{4 F_{0}^{2}}$ & $\frac{g_{2} g_{3} g_{5}}{2 F_{0}^{2}}$ & $-\frac{g_{4} g_{5}^{2}}{2 F_{0}^{2}}$ \\
\hline & $\eta$-loop & - & - & - & - \\
\hline & $\pi$-loop & $\frac{2 g_{2}^{2} g_{4}}{F_{0}^{2}}-\frac{2 g_{1} g_{2} g_{3}}{F_{0}^{2}}$ & $\frac{g_{3}^{2} g_{4}}{2 F_{0}^{2}}-\frac{g_{4}^{3}}{2 F_{0}^{2}}$ & $\frac{2 g_{2} g_{3} g_{5}}{F_{0}^{2}}$ & $-\frac{2 g_{4} g_{5}^{2}}{F_{0}^{2}}$ \\
\hline \multirow{3}{*}{$g_{\Xi_{c}^{+} \Xi_{c}^{* \prime \prime}}$} & $K$-loop & $\frac{\sqrt{2} g_{2}^{2} g_{4}}{F_{0}^{2}}-\frac{\sqrt{2} g_{1} g_{2} g_{3}}{F_{0}^{2}}$ & $\frac{g_{3}^{2} g_{4}}{2 \sqrt{2} F_{0}^{2}}-\frac{g_{4}^{3}}{2 \sqrt{2} F_{0}^{2}}$ & $\frac{\sqrt{2} g_{2} g_{3} g_{5}}{F^{2}}$ & $-\frac{\sqrt{2} g_{4} g_{5}^{2}}{F^{2}}$ \\
\hline & $\eta$-loop & $\frac{3 g_{2}^{2} g_{4}}{2 \sqrt{2} F_{0}^{2}}-\frac{g_{1} g_{2} g_{3}}{4 \sqrt{2} F_{0}^{2}}$ & $\frac{g_{3}^{2} g_{4}}{16 \sqrt{2} F_{0}^{2}}-\frac{3 g_{4}^{3}}{8 \sqrt{2} F_{0}^{2}}$ & $\frac{g_{2} g_{3} g_{5}}{4 \sqrt{2} F_{0}^{2}}$ & $-\frac{g_{4} g_{5}^{2}}{4 \sqrt{2} F_{0}^{2}}$ \\
\hline & $\pi$-loop & $-\frac{g_{4} g_{2}^{2}}{2 \sqrt{2} F_{0}^{2}}-\frac{g_{1} g_{3} g_{2}}{4 \sqrt{2} F_{0}^{2}}$ & $\frac{g_{4}^{3}}{8 \sqrt{2} F_{0}^{2}}+\frac{g_{3}^{2} g_{4}}{16 \sqrt{2} F_{0}^{2}}$ & $\frac{g_{2} g_{3} g_{5}}{4 \sqrt{2} F_{0}^{2}}$ & $-\frac{g_{4} g_{5}^{2}}{4 \sqrt{2} F_{0}^{2}}$ \\
\hline \multirow{3}{*}{$g_{\Lambda_{c}^{+} \Xi_{c}^{* \prime 0}}$} & $K$-loop & $-\frac{g_{1} g_{2} g_{3}}{\sqrt{2} F_{0}^{2}}$ & $\frac{g_{3}^{2} g_{4}}{4 \sqrt{2} F_{0}^{2}}$ & $\frac{g_{2} g_{3} g_{5}}{\sqrt{2} F_{0}^{2}}$ & $-\frac{g_{4} g_{5}^{2}}{\sqrt{2} F_{0}^{2}}$ \\
\hline & $\eta$-loop & - & - & - & - \\
\hline & $\pi$-loop & $\frac{3 g_{2}^{2} g_{4}}{\sqrt{2} F_{0}^{2}}-\frac{3 g_{1} g_{2} g_{3}}{2 \sqrt{2} F_{0}^{2}}$ & $\frac{3 g_{3}^{2} g_{4}}{8 \sqrt{2} F_{0}^{2}}-\frac{3 g_{4}^{3}}{4 \sqrt{2} F_{0}^{2}}$ & $\frac{3 g_{2} g_{3} g_{5}}{2 \sqrt{2} F_{0}^{2}}$ & $-\frac{3 g_{4} g_{5}^{2}}{2 \sqrt{2} F_{0}^{2}}$ \\
\hline \multirow{3}{*}{$g_{\Xi_{c}^{+} \Omega_{c}^{* 0}}$} & $K$-loop & $\frac{3 g_{2}^{2} g_{4}}{F_{0}^{2}}-\frac{3 g_{1} g_{2} g_{3}}{2 F_{0}^{2}}$ & $\frac{3 g_{3}^{2} g_{4}}{8 F_{0}^{2}}-\frac{3 g_{4}^{3}}{4 F_{0}^{2}}$ & $\frac{3 g_{2} g_{3} g_{5}}{2 F_{0}^{2}}$ & $-\frac{3 g_{4} g_{5}^{2}}{2 F_{0}^{2}}$ \\
\hline & $\eta$-loop & $-\frac{g_{1} g_{2} g_{3}}{F_{0}^{2}}$ & $\frac{g_{3}^{2} g_{4}}{4 F_{0}^{2}}$ & $\frac{g_{2} g_{3} g_{5}}{F_{0}^{2}}$ & $-\frac{g_{4} g_{5}^{2}}{F_{0}^{2}}$ \\
\hline & $\pi$-loop & - & - & - & - \\
\hline
\end{tabular}

TABLE XIII: The coefficients $g_{(i j)}^{b}$ of the axial current from diagram (b).

\begin{tabular}{|c|c|c|c|c|c|c|c|}
\hline & $K$-loop & $\eta$-loop & $\pi$-loop & $g_{\Sigma_{c}^{*+} \Xi_{c}^{* \prime 0}}$ & $-\frac{3 g_{5}}{4 F_{0}^{2}}$ & $-\frac{5 g_{5}}{24 F_{0}^{2}}$ & $-\frac{3 g_{5}}{8 F_{0}^{2}}$ \\
\hline$g_{\Xi_{c}^{\prime+} \Xi_{c}^{\prime 0}}$ & $-\frac{g_{1}}{2 F_{0}^{2}}$ & $-\frac{g_{1}}{12 F_{0}^{2}}$ & $-\frac{3 g_{1}}{4 F_{0}^{2}}$ & $g_{\Sigma_{c}^{*++} \Xi_{c}^{* \prime+}}$ & $\frac{395}{2 \sqrt{2} F_{0}^{2}}$ & $\frac{5 g_{5}}{12 \sqrt{2} F_{0}^{2}}$ & $-\frac{3 g_{5}}{4 \sqrt{2} F_{0}^{2}}$ \\
\hline$g_{\Sigma_{c}^{+} \Sigma_{c}^{0}}$ & $-\frac{g_{1}}{\sqrt{2} F_{0}^{2}}$ & $-\frac{g_{1}}{6 \sqrt{2} F_{0}^{2}}$ & $-\frac{3 g_{1}}{2 \sqrt{2} F_{0}^{2}}$ & $g_{\Xi_{c}^{\prime+} \Xi_{c}^{* \prime 0}}$ & $-\frac{g_{3}}{4 F_{0}^{2}}$ & $-\frac{g_{3}}{24 F_{0}^{2}}$ & $-\frac{3 g_{3}}{8 F_{0}^{2}}$ \\
\hline$g_{\Xi_{c}^{\prime+} \Omega_{c}^{0}}$ & $-\frac{3 g_{1}}{2 \sqrt{2} F_{0}^{2}}$ & $-\frac{5 g_{1}}{12 \sqrt{2} F_{0}^{2}}$ & $-\frac{3 g_{1}}{4 \sqrt{2} F_{0}^{2}}$ & $g_{\Sigma_{c}^{+} \Sigma_{c}^{* 0}}$ & $-\frac{g_{3}}{2 \sqrt{2} F_{0}^{2}}$ & $-\frac{g_{3}}{12 \sqrt{2} F_{0}^{2}}$ & $-\frac{3 g_{3}}{4 \sqrt{2} F_{0}^{2}}$ \\
\hline$g_{\Sigma_{c}^{+} \Xi_{c}^{\prime 0}}$ & $-\frac{3 g_{1}}{4 F_{0}^{2}}$ & $-\frac{5 g_{1}}{24 F_{0}^{2}}$ & $-\frac{3 g_{1}}{8 F_{0}^{2}}$ & $g_{\Sigma_{c}^{++} \Sigma_{c}^{*+}}$ & $-\frac{g_{3}}{2 \sqrt{2} F_{0}^{2}}$ & $-\frac{g_{3}}{12 \sqrt{2} F_{0}^{2}}$ & $-\frac{3 g_{3}}{4 \sqrt{2} F_{0}^{2}}$ \\
\hline$g_{\Sigma_{c}^{++} \Xi_{c}^{\prime+}}$ & $-\frac{3 g_{1}}{2 \sqrt{2} F_{0}^{2}}$ & $-\frac{5 g_{1}}{12 \sqrt{2} F_{0}^{2}}$ & $-\frac{3 g_{1}}{4 \sqrt{2} F_{0}^{2}}$ & $g_{\Xi_{c}^{\prime+} \Omega_{c}^{* 0}}$ & $-\frac{3 g_{3}}{4 \sqrt{2} F_{0}^{2}}$ & $-\frac{5 g_{3}}{24 \sqrt{2} F_{0}^{2}}$ & $-\frac{3 g_{3}}{8 \sqrt{2} F_{0}^{2}}$ \\
\hline$g_{\Lambda_{c}^{+} \Sigma_{c}^{0}}$ & $-\frac{g_{2}}{F_{0}^{2}}$ & $-\frac{g_{2}}{6 F_{0}^{2}}$ & $-\frac{3 g_{2}}{2 F_{0}^{2}}$ & $g_{\Sigma_{c}^{+} \Xi_{c}^{* \prime \prime}}$ & $-\frac{3 g_{3}}{8 F_{0}^{2}}$ & $-\frac{5 g_{3}}{48 F_{0}^{2}}$ & $-\frac{3 g_{3}}{16 F_{0}^{2}}$ \\
\hline$g_{\Xi_{c}^{+} \Xi_{c}^{\prime 0}}$ & $-\frac{g_{2}}{\sqrt{2} F_{0}^{2}}$ & $-\frac{g_{2}}{6 \sqrt{2} F_{0}^{2}}$ & $-\frac{3 g_{2}}{2 \sqrt{2} F_{0}^{2}}$ & $g_{\Sigma_{c}^{++} \Xi_{c}^{* \prime+}}$ & $-\frac{3 g_{3}}{4 \sqrt{2} F_{0}^{2}}$ & $-\frac{5 g_{3}}{24 \sqrt{2} F_{0}^{2}}$ & $-\frac{3 g_{3}}{8 \sqrt{2} F_{0}^{2}}$ \\
\hline$g_{\Lambda_{c}^{+} \Xi_{c}^{\prime 0}}$ & $-\frac{3 g_{2}}{2 \sqrt{2} F_{0}^{2}}$ & $-\frac{5 g_{2}}{12 \sqrt{2} F_{0}^{2}}$ & $-\frac{3 g_{2}}{4 \sqrt{2} F_{0}^{2}}$ & $g_{\Xi_{c}^{* \prime}+\Omega_{c}^{0}}$ & $-\frac{3 g_{3}}{4 \sqrt{2} F_{0}^{2}}$ & $-\frac{5 g_{3}}{24 \sqrt{2} F_{0}^{2}}$ & $-\frac{3 g_{3}}{8 \sqrt{2} F_{0}^{2}}$ \\
\hline$g_{\Xi_{c}^{+} \Omega_{c}^{0}}$ & $-\frac{3 g_{2}}{2 F_{0}^{2}}$ & $-\frac{5 g_{2}}{12 F_{0}^{2}}$ & $-\frac{3 g_{2}}{4 F_{0}^{2}}$ & $g_{\Sigma_{c}^{*+} \Xi_{c}^{\prime 0}}$ & $-\frac{3 g_{3}}{8 F_{0}^{2}}$ & $-\frac{5 g_{3}}{48 F_{0}^{2}}$ & $-\frac{3 g_{3}}{16 F_{0}^{2}}$ \\
\hline$g_{\Sigma_{c}^{+} \Xi_{c}^{0}}$ & $\frac{3 g_{2}}{2 \sqrt{2} F_{0}^{2}}$ & $\frac{5 g_{2}}{12 \sqrt{2} F_{0}^{2}}$ & $\frac{3 g_{2}}{4 \sqrt{2} F_{0}^{2}}$ & $\underline{g_{\Sigma_{c}^{*++}} \Xi_{c}^{\prime+}}$ & $-\frac{3 g_{3}}{4 \sqrt{2} F_{0}^{2}}$ & $\frac{5 g_{3}}{24 \sqrt{2} F_{0}^{2}}$ & $-\frac{3 g_{3}}{8 \sqrt{2} F_{0}^{2}}$ \\
\hline$g_{\Sigma_{c}^{++}} \Xi_{c}^{+}$ & $\frac{3 g_{2}}{2 F_{0}^{2}}$ & $\frac{5 g_{2}}{12 F_{0}^{2}}$ & $\frac{3 g_{2}}{4 F_{0}^{2}}$ & $g_{\Lambda_{c}^{+} \Sigma_{c}^{* 0}}$ & $-\frac{g_{4}}{2 F_{0}^{2}}$ & $-\frac{g_{4}}{12 F_{0}^{2}}$ & $-\frac{3 g_{4}}{4 F_{0}^{2}}$ \\
\hline$g_{\Xi_{c}^{* \prime}+\Xi_{c}^{* \prime 0}}$ & $-\frac{g_{5}}{2 F_{0}^{2}}$ & $-\frac{g_{5}}{12 F_{0}^{2}}$ & $-\frac{3 g_{5}}{4 F_{0}^{2}}$ & $g_{\Xi_{c}^{+} \Xi_{c}^{* \prime 0}}$ & $-\frac{g_{4}}{2 \sqrt{2} F_{0}^{2}}$ & $-\frac{g_{4}}{12 \sqrt{2} F_{0}^{2}}$ & $-\frac{3 g_{4}}{4 \sqrt{2} F_{0}^{2}}$ \\
\hline$g_{\Sigma_{c}^{*+} \Sigma_{c}^{* 0}}$ & $-\frac{g_{5}}{\sqrt{2} F_{0}^{2}}$ & $-\frac{g_{5}}{6 \sqrt{2} F_{0}^{2}}$ & $-\frac{3 g_{5}}{2 \sqrt{2} F_{0}^{2}}$ & $g_{\Lambda_{c}^{+} \Xi_{c}^{* \prime 0}}$ & $-\frac{3 g_{4}}{4 \sqrt{2} F_{0}^{2}}$ & $-\frac{5 g_{4}}{24 \sqrt{2} F_{0}^{2}}$ & $-\frac{3 g_{4}}{8 \sqrt{2} F_{0}^{2}}$ \\
\hline$g_{\Xi_{c}^{* \prime+}+\Omega_{c}^{* 0}}$ & $-\frac{3 g_{5}}{2 \sqrt{2} F_{0}^{2}}$ & $-\frac{5 g_{5}}{12 \sqrt{2} F_{0}^{2}}$ & $-\frac{3 g_{5}}{4 \sqrt{2} F_{0}^{2}}$ & $g_{\Xi_{c}^{+} \Omega_{c}^{* 0}}$ & $-\frac{3 g_{4}}{4 F_{0}^{2}}$ & $-\frac{5 g_{4}}{24 F_{0}^{2}}$ & $-\frac{3 g_{4}}{8 F_{0}^{2}}$ \\
\hline
\end{tabular}


TABLE XIV: The coefficients $\lambda_{1(i j)}$ of the axial current from the wave function renormalization.

\begin{tabular}{l|lll}
\hline & & Case I & Case II \\
\hline \hline$\lambda_{\Xi_{c}^{\prime+} \Xi_{c}^{\prime 0}}$ & K-loop & $\frac{5 g_{1}^{2}}{2 F_{0}^{2}}+\frac{g_{2}^{2}}{F_{0}^{2}}$ & $-\frac{5 g_{3}^{2}}{8 F_{0}^{2}}$ \\
& $\eta$-loop & $\frac{g_{1}^{2}}{12 F_{0}^{2}}+\frac{3 g_{2}^{2}}{2 F_{0}^{2}}$ & $-\frac{g_{3}^{2}}{48 F_{0}^{2}}$ \\
& $\pi$-loop & $\frac{3 g_{1}^{2}}{4 F_{0}^{2}}+\frac{3 g_{2}^{2}}{2 F_{0}^{2}}$ & $-\frac{3 g_{3}^{2}}{16 F_{0}^{2}}$ \\
\hline$\lambda_{\Sigma_{c}^{+} \Sigma_{c}^{0}}$ & $K$-loop & $\frac{g_{1}^{2}}{F_{0}^{2}}+\frac{2 g_{2}^{2}}{F_{0}^{2}}$ & $-\frac{g_{3}^{2}}{4 F_{0}^{2}}$ \\
& $\eta$-loop & $\frac{g_{1}^{2}}{3 F_{0}^{2}}$ & $-\frac{g_{3}^{2}}{12 F_{0}^{2}}$ \\
& $\pi$-loop & $\frac{2 g_{1}^{2}}{F_{0}^{2}}+\frac{2 g_{2}^{2}}{F_{0}^{2}}$ & $-\frac{g_{3}^{2}}{2 F_{0}^{2}}$ \\
\hline$\lambda_{\Xi_{c}^{\prime+} \Omega_{c}^{0}}$ & K-loop & $\frac{9 g_{1}^{2}}{4 F_{0}^{2}}+\frac{5 g_{2}^{2}}{2 F_{0}^{2}}$ & $-\frac{9 g_{3}^{2}}{16 F_{0}^{2}}$ \\
& $\eta$-loop & $\frac{17 g_{1}^{2}}{24 F_{0}^{2}}+\frac{3 g_{2}^{2}}{4 F_{0}^{2}}$ & $-\frac{17 g_{3}^{2}}{96 F_{0}^{2}}$ \\
& $\pi$-loop & $\frac{3 g_{1}^{2}}{8 F_{0}^{2}}+\frac{3 g_{2}^{2}}{4 F_{0}^{2}}$ & $-\frac{3 g_{3}^{2}}{32 F_{0}^{2}}$ \\
\hline$\lambda_{\Sigma_{c}^{+} \Xi_{c}^{\prime 0}}$ & $K$-loop & $\frac{7 g_{1}^{2}}{4 F_{0}^{2}}+\frac{3 g_{2}^{2}}{2 F_{0}^{2}}$ & $-\frac{7 g_{3}^{2}}{16 F_{0}^{2}}$ \\
& $\eta$-loop & $\frac{5 g_{1}^{2}}{24 F_{0}^{2}}+\frac{3 g_{2}^{2}}{4 F_{0}^{2}}$ & $-\frac{5 g_{3}^{2}}{96 F_{0}^{2}}$ \\
& $\pi$-loop & $\frac{11 g_{1}^{2}}{8 F_{0}^{2}}+\frac{7 g_{2}^{2}}{4 F_{0}^{2}}$ & $-\frac{11 g_{3}^{2}}{32 F_{0}^{2}}$ \\
\hline$\Sigma_{\Sigma_{c}^{++} \Xi_{c}^{\prime+}}$ & K-loop & $\frac{7 g_{1}^{2}}{4 F_{0}^{2}}+\frac{3 g_{2}^{2}}{2 F_{0}^{2}}$ & $-\frac{7 g_{3}^{2}}{16 F_{0}^{2}}$ \\
& $\eta$-loop & $\frac{5 g_{1}^{2}}{24 F_{0}^{2}}+\frac{3 g_{2}^{2}}{4 F_{0}^{2}}$ & $-\frac{5 g_{3}^{2}}{96 F_{0}^{2}}$ \\
& $\pi$-loop & $\frac{11 g_{1}^{2}}{8 F_{0}^{2}}+\frac{7 g_{2}^{2}}{4 F_{0}^{2}}$ & $-\frac{11 g_{3}^{2}}{32 F_{0}^{2}}$ \\
\hline
\end{tabular}

TABLE XV: The coefficients $\lambda_{2(i j)}$ of the axial current from the wave function renormalization.

\begin{tabular}{|c|c|c|c|}
\hline & & I & II \\
\hline$\lambda_{\Lambda_{c}^{+} \Sigma_{c}^{0}}$ & $\begin{array}{l}K \text {-loop } \\
\eta \text {-loop } \\
\pi \text {-loop }\end{array}$ & $\begin{array}{c}\frac{g_{1}^{2}}{2 F_{0}^{2}}+\frac{2 g_{2}^{2}}{F_{0}^{2}}+\frac{2 g_{6}^{2}}{F_{0}^{2}} \\
\frac{g_{1}^{2}}{6 F_{0}^{2}}+\frac{2 g_{6}^{2}}{3 F_{0}^{2}} \\
\frac{g_{1}^{2}}{F_{0}^{2}}+\frac{4 g_{2}^{2}}{F_{0}^{2}}\end{array}$ & $\begin{array}{c}-\frac{g_{3}^{2}}{8 F_{0}^{2}}-\frac{g_{4}^{2}}{4 F_{0}^{2}} \\
-\frac{g_{3}^{2}}{24 F_{0}^{2}} \\
-\frac{g_{3}^{2}}{4 F_{0}^{2}}-\frac{3 g_{4}^{2}}{4 F_{0}^{2}}\end{array}$ \\
\hline$\lambda_{\Xi_{c}^{+} \Xi_{c}^{\prime 0}}$ & $\begin{array}{l}K \text {-loop } \\
\eta \text {-loop } \\
\pi \text {-loop }\end{array}$ & $\begin{array}{c}\frac{5 g_{1}^{2}}{4 F_{0}^{2}}+\frac{3 g_{2}^{2}}{F_{0}^{2}}+\frac{g_{6}^{2}}{F_{0}^{2}} \\
\frac{g_{1}^{2}}{24 F_{0}^{2}}+\frac{3 g_{2}^{2}}{2 F_{0}^{2}}+\frac{g_{6}^{2}}{6 F_{0}^{2}} \\
\frac{3 g_{1}^{2}}{8 F_{0}^{2}}+\frac{3 g_{2}^{2}}{2 F_{0}^{2}}+\frac{3 g_{6}^{2}}{2 F_{0}^{2}}\end{array}$ & $\begin{array}{l}-\frac{5 g_{3}^{2}}{16 F_{0}^{2}}-\frac{5 g_{4}^{2}}{8 F_{0}^{2}} \\
-\frac{g_{3}^{2}}{96 F_{0}^{2}}-\frac{3 g_{4}^{2}}{16 F_{0}^{2}} \\
-\frac{3 g_{3}^{2}}{32 F_{0}^{2}}-\frac{3 g_{4}^{2}}{16 F_{0}^{2}}\end{array}$ \\
\hline$\lambda_{\Lambda}$ & $\begin{array}{l}K \text {-loop } \\
\eta \text {-loop } \\
\pi \text {-loop }\end{array}$ & $\begin{array}{c}\frac{5 g_{1}^{2}}{4 F_{0}^{2}}+\frac{3 g_{2}^{2}}{2 F_{0}^{2}}+\frac{2 g_{6}^{2}}{F_{0}^{2}} \\
\frac{g_{1}^{2}}{24 F_{0}^{2}}+\frac{3 g_{2}^{2}}{4 F_{0}^{2}}+\frac{2 g_{6}^{2}}{3 F_{0}^{2}} \\
\frac{3 g_{1}^{2}}{8 F_{0}^{2}}+\frac{15 g_{2}^{2}}{4 F_{0}^{2}}\end{array}$ & $\begin{array}{c}-\frac{5 g_{3}^{2}}{16 F_{0}^{2}}-\frac{g_{4}^{2}}{4 F_{0}^{2}} \\
\quad-\frac{g_{3}^{2}}{96 F_{0}^{2}} \\
-\frac{3 g_{3}^{2}}{32 F_{0}^{2}}-\frac{3 g_{4}^{2}}{4 F_{0}^{2}}\end{array}$ \\
\hline$\lambda_{\Xi_{c}^{+} \Omega_{c}^{0}}$ & $\begin{array}{l}K \text {-loop } \\
\eta \text {-loop } \\
\pi \text {-loop }\end{array}$ & $\begin{array}{c}\frac{g_{1}^{2}}{F_{0}^{2}}+\frac{9 g_{2}^{2}}{2 F_{0}^{2}}+\frac{g_{6}^{2}}{F_{0}^{2}} \\
\frac{2 g_{1}^{2}}{3 F_{0}^{2}}+\frac{3 g_{2}^{2}}{4 F_{0}^{2}}+\frac{g_{6}^{2}}{6 F_{0}^{2}} \\
\frac{3 g_{2}^{2}}{4 F_{0}^{2}}+\frac{3 g_{6}^{2}}{2 F_{0}^{2}}\end{array}$ & $\begin{array}{c}-\frac{g_{3}^{2}}{4 F_{0}^{2}}-\frac{5 g_{4}^{2}}{8 F_{0}^{2}} \\
-\frac{g_{3}^{2}}{6 F_{0}^{2}}-\frac{3 g_{4}^{2}}{16 F_{0}^{2}} \\
-\frac{3 g_{4}^{2}}{16 F_{0}^{2}} \\
\end{array}$ \\
\hline$\lambda_{\Sigma_{c}^{+} \Xi_{c}^{+}}$ & $\begin{array}{l}K \text {-loop } \\
\eta \text {-loop } \\
\pi \text {-loop }\end{array}$ & $\begin{array}{l}\frac{g_{1}^{2}}{2 F_{0}^{2}}+\frac{7 g_{2}^{2}}{2 F_{0}^{2}}+\frac{g_{6}^{2}}{F_{0}^{2}} \\
\frac{g_{1}^{2}}{6 F_{0}^{2}}+\frac{3 g_{2}^{2}}{4 F_{0}^{2}}+\frac{g_{6}^{2}}{6 F_{0}^{2}} \\
\frac{g_{1}^{2}}{F_{0}^{2}}+\frac{7 g_{2}^{2}}{4 F_{0}^{2}}+\frac{3 g_{6}^{2}}{2 F_{0}^{2}}\end{array}$ & $\begin{array}{r}-\frac{g_{3}^{2}}{8 F_{0}^{2}}-\frac{5 g_{4}^{2}}{8 F_{0}^{2}} \\
-\frac{g_{3}^{2}}{24 F_{0}^{2}}-\frac{3 g_{4}^{2}}{16 F_{0}^{2}} \\
-\frac{g_{3}^{2}}{4 F_{0}^{2}}-\frac{3 g_{4}^{2}}{16 F_{0}^{2}}\end{array}$ \\
\hline$\lambda_{\Sigma_{c}^{++} \Xi_{c}^{+}}$ & $\begin{array}{l}K \text {-loop } \\
\eta \text {-loop } \\
\pi \text {-loop }\end{array}$ & $\begin{array}{l}\frac{g_{1}^{2}}{2 F_{0}^{2}}+\frac{7 g_{2}^{2}}{2 F_{0}^{2}}+\frac{g_{6}^{2}}{F_{0}^{2}} \\
\frac{g_{1}^{2}}{6 F_{0}^{2}}+\frac{3 g_{2}^{2}}{4 F_{0}^{2}}+\frac{g_{6}^{2}}{6 F_{0}^{2}} \\
\frac{g_{1}^{2}}{F_{0}^{2}}+\frac{7 g_{2}^{2}}{4 F_{0}^{2}}+\frac{3 g_{6}^{2}}{2 F_{0}^{2}}\end{array}$ & $\begin{array}{c}-\frac{g_{3}^{2}}{8 F_{0}^{2}}-\frac{5 g_{4}^{2}}{8 F_{0}^{2}} \\
-\frac{g_{3}^{2}}{24 F_{0}^{2}}-\frac{3 g_{4}^{2}}{16 F_{0}^{2}} \\
-\frac{g_{3}^{2}}{4 F_{0}^{2}}-\frac{3 g_{4}^{2}}{16 F_{0}^{2}}\end{array}$ \\
\hline
\end{tabular}


TABLE XVI: The coefficients $\lambda_{5(i j)}$ of the axial current from the wave function renormalization.

\begin{tabular}{|c|c|c|c|}
\hline & & Case I & Case II \\
\hline$\lambda_{\Xi_{c}^{* \prime+}} \Xi_{c}^{* \prime 0}$ & $\begin{array}{l}K \text {-loop } \\
\eta \text {-loop } \\
\pi \text {-loop } \\
\end{array}$ & $\begin{array}{l}\frac{5 g_{5}^{2}}{2 F_{0}^{2}} \\
\frac{g_{5}^{2}}{12 F_{0}^{2}} \\
\frac{3 g_{5}^{2}}{4 F_{0}^{2}} \\
\end{array}$ & $\begin{array}{l}-\frac{5 g_{3}^{2}}{8 F_{0}^{2}}-\frac{g_{4}^{2}}{4 F_{0}^{2}} \\
-\frac{g_{3}^{2}}{48 F_{0}^{2}}-\frac{3 g_{4}^{2}}{8 F_{0}^{2}} \\
-\frac{3 g_{3}^{2}}{16 F_{0}^{2}}-\frac{3 g_{4}^{2}}{8 F_{0}^{2}}\end{array}$ \\
\hline$\lambda_{\Sigma_{c}^{*}}$ & $\begin{array}{l}K \text {-loop } \\
\eta \text {-loop } \\
\pi \text {-loop } \\
\end{array}$ & $\begin{array}{l}\frac{g_{5}^{2}}{F_{0}^{2}} \\
\frac{g_{5}^{2}}{3 F_{0}^{2}} \\
\frac{2 g_{5}^{2}}{F_{0}^{2}} \\
\end{array}$ & $\begin{array}{c}-\frac{g_{3}^{2}}{4 F_{0}^{2}}-\frac{g_{4}^{2}}{2 F_{0}^{2}} \\
-\frac{g_{3}^{2}}{12 F_{0}^{2}} \\
-\frac{g_{3}^{2}}{2 F_{0}^{2}}-\frac{g_{4}^{2}}{2 F_{0}^{2}}\end{array}$ \\
\hline$\lambda_{\Xi_{c}^{*}}$ & $\begin{array}{l}K \text {-loop } \\
\eta \text {-loop } \\
\pi \text {-loop }\end{array}$ & $\begin{array}{l}\frac{9 g_{5}^{2}}{4 F_{0}^{2}} \\
\frac{17 g_{5}^{2}}{24 F_{0}^{2}} \\
\frac{3 g_{5}^{2}}{8 F_{0}^{2}} \\
\end{array}$ & $\begin{array}{l}-\frac{9 g_{3}^{2}}{16 F_{0}^{2}}-\frac{5 g_{4}^{2}}{8 F_{0}^{2}} \\
-\frac{17 g_{3}^{2}}{96 F_{0}^{2}}-\frac{3 g_{4}^{2}}{16 F_{0}^{2}} \\
-\frac{3 g_{3}^{2}}{32 F_{0}^{2}}-\frac{3 g_{4}^{2}}{16 F_{0}^{2}}\end{array}$ \\
\hline$E_{c}^{* \prime 0}$ & $\begin{array}{l}K \text {-loop } \\
\eta \text {-loop } \\
\pi \text {-loop }\end{array}$ & $\begin{array}{l}\frac{7 g_{5}^{2}}{4 F_{0}^{2}} \\
\frac{5 g_{5}^{2}}{24 F_{0}^{2}} \\
\frac{11 g_{5}^{2}}{8 F_{0}^{2}}\end{array}$ & $\begin{array}{l}-\frac{7 g_{3}^{2}}{16 F_{0}^{2}}-\frac{3 g_{4}^{2}}{8 F_{0}^{2}} \\
-\frac{5 g_{3}^{2}}{96 F_{0}^{2}}-\frac{3 g_{4}^{2}}{16 F_{0}^{2}} \\
-\frac{11 g_{3}^{2}}{32 F_{0}^{2}}-\frac{7 g_{4}^{2}}{16 F_{0}^{2}}\end{array}$ \\
\hline$\lambda_{\Sigma_{c}^{*++} \Xi_{c}^{* \prime+}}$ & $\begin{array}{l}K \text {-loop } \\
\eta \text {-loop } \\
\pi \text {-loop }\end{array}$ & $\begin{array}{l}\frac{7 g_{5}^{2}}{4 F_{0}^{2}} \\
\frac{5 g_{5}^{2}}{24 F_{0}^{2}} \\
\frac{11 g_{5}^{2}}{8 F_{0}^{2}} \\
\end{array}$ & $\begin{array}{l}-\frac{7 g_{3}^{2}}{16 F_{0}^{2}}-\frac{3 g_{4}^{2}}{8 F_{0}^{2}} \\
-\frac{5 g_{3}^{2}}{96 F_{0}^{2}}-\frac{3 g_{4}^{2}}{16 F_{0}^{2}} \\
-\frac{11 g_{3}^{2}}{32 F_{0}^{2}}-\frac{7 g_{4}^{2}}{16 F_{0}^{2}}\end{array}$ \\
\hline
\end{tabular}


TABLE XVII: The coefficients $\lambda_{3(i j)}$ of the axial current from the wave function renormalization.

\begin{tabular}{|c|c|c|c|}
\hline & & Case I & Case II \\
\hline$\lambda_{\Xi_{c}^{\prime+} \Xi_{c}^{* \prime 0}}$ & $\begin{array}{l}K \text {-loop } \\
\eta \text {-loop } \\
\pi \text {-loop }\end{array}$ & $\begin{array}{l}\frac{5 g_{1}^{2}}{4 F_{0}^{2}}+\frac{g_{2}^{2}}{2 F_{0}^{2}}+\frac{5 g_{5}^{2}}{4 F_{0}^{2}} \\
\frac{g_{1}^{2}}{24 F_{0}^{2}}+\frac{3 g_{2}^{2}}{4 F_{0}^{2}}+\frac{g_{5}^{2}}{24 F_{0}^{2}} \\
\frac{3 g_{1}^{2}}{8 F_{0}^{2}}+\frac{3 g_{2}^{2}}{4 F_{0}^{2}}+\frac{3 g_{5}^{2}}{8 F_{0}^{2}}\end{array}$ & $\begin{array}{r}-\frac{5 g_{3}^{2}}{8 F_{0}^{2}}-\frac{g_{4}^{2}}{8 F_{0}^{2}} \\
-\frac{g_{3}^{2}}{48 F_{0}^{2}}-\frac{3 g_{4}^{2}}{16 F_{0}^{2}} \\
-\frac{3 g_{3}^{2}}{16 F_{0}^{2}}-\frac{3 g_{4}^{2}}{16 F_{0}^{2}}\end{array}$ \\
\hline$\lambda_{\Sigma_{c}^{+} \Sigma_{c}^{* 0}}$ & $\begin{array}{c}K \text {-loop } \\
\eta \text {-loop } \\
\pi \text {-loop }\end{array}$ & $\begin{array}{c}\frac{g_{1}^{2}}{2 F_{0}^{2}}+\frac{g_{2}^{2}}{F_{0}^{2}}+\frac{g_{5}^{2}}{2 F_{0}^{2}} \\
\frac{g_{1}^{2}}{6 F_{0}^{2}}+\frac{g_{5}^{2}}{6 F_{0}^{2}} \\
\frac{g_{1}^{2}}{F_{0}^{2}}+\frac{g_{2}^{2}}{F_{0}^{2}}+\frac{g_{5}^{2}}{F_{0}^{2}}\end{array}$ & $\begin{array}{c}-\frac{g_{3}^{2}}{4 F_{0}^{2}}-\frac{g_{4}^{2}}{4 F_{0}^{2}} \\
-\frac{g_{3}^{2}}{12 F_{0}^{2}} \\
-\frac{g_{3}^{2}}{2 F_{0}^{2}}-\frac{g_{4}^{2}}{4 F_{0}^{2}}\end{array}$ \\
\hline$\lambda_{\Sigma_{c}^{++} \Sigma_{c}^{*+}}$ & $\begin{array}{l}K \text {-loop } \\
\eta \text {-loop } \\
\pi \text {-loop }\end{array}$ & $\begin{array}{c}\frac{g_{1}^{2}}{2 F_{0}^{2}}+\frac{g_{2}^{2}}{F_{0}^{2}}+\frac{g_{5}^{2}}{2 F_{0}^{2}} \\
\frac{g_{1}^{2}}{6 F_{0}^{2}}+\frac{g_{5}^{2}}{6 F_{0}^{2}} \\
\frac{g_{1}^{2}}{F_{0}^{2}}+\frac{g_{2}^{2}}{F_{0}^{2}}+\frac{g_{5}^{2}}{F_{0}^{2}} \\
\end{array}$ & $\begin{array}{c}-\frac{g_{3}^{2}}{4 F_{0}^{2}}-\frac{g_{4}^{2}}{4 F_{0}^{2}} \\
-\frac{g_{3}^{2}}{12 F_{0}^{2}} \\
-\frac{g_{3}^{2}}{2 F_{0}^{2}}-\frac{g_{4}^{2}}{4 F_{0}^{2}} \\
\end{array}$ \\
\hline$\lambda_{\Xi_{c}{ }^{\prime}}$ & $\begin{array}{c}K \text {-loop } \\
\eta \text {-loop } \\
\pi \text {-loop }\end{array}$ & $\begin{array}{c}\frac{5 g_{1}^{2}}{4 F_{0}^{2}}+\frac{g_{2}^{2}}{2 F_{0}^{2}}+\frac{g_{5}^{2}}{F_{0}^{2}} \\
\frac{g_{1}^{2}}{24 F_{0}^{2}}+\frac{3 g_{2}^{2}}{4 F_{0}^{2}}+\frac{2 g_{5}^{2}}{3 F_{0}^{2}} \\
\frac{3 g_{1}^{2}}{8 F_{0}^{2}}+\frac{3 g_{2}^{2}}{4 F_{0}^{2}} \\
\end{array}$ & $\begin{array}{c}-\frac{9 g_{3}^{2}}{16 F_{0}^{2}}-\frac{g_{4}^{2}}{2 F_{0}^{2}} \\
-\frac{17 g_{3}^{2}}{96 F_{0}^{2}} \\
-\frac{3 g_{3}^{2}}{32 F_{0}^{2}} \\
\end{array}$ \\
\hline$\lambda_{\Sigma_{c}^{+} \Xi_{c}^{* \prime+}}$ & $\begin{array}{l}K \text {-loop } \\
\eta \text {-loop } \\
\pi \text {-loop }\end{array}$ & $\begin{array}{c}\frac{g_{1}^{2}}{2 F_{0}^{2}}+\frac{g_{2}^{2}}{F_{0}^{2}}+\frac{5 g_{5}^{2}}{4 F_{0}^{2}} \\
\quad \frac{g_{1}^{2}}{6 F_{0}^{2}}+\frac{g_{5}^{2}}{24 F_{0}^{2}} \\
\frac{g_{1}^{2}}{F_{0}^{2}}+\frac{g_{2}^{2}}{F_{0}^{2}}+\frac{3 g_{5}^{2}}{8 F_{0}^{2}} \\
\end{array}$ & $\begin{array}{l}-\frac{7 g_{3}^{2}}{16 F_{0}^{2}}-\frac{g_{4}^{2}}{8 F_{0}^{2}} \\
-\frac{5 g_{3}^{2}}{96 F_{0}^{2}}-\frac{3 g_{4}^{2}}{16 F_{0}^{2}} \\
-\frac{11 g_{3}^{2}}{32 F_{0}^{2}}-\frac{3 g_{4}^{2}}{16 F_{0}^{2}}\end{array}$ \\
\hline$\lambda_{\Sigma_{c}^{++}} \Xi_{c}^{* \prime+}$ & $\begin{array}{l}K \text {-loop } \\
\eta \text {-loop } \\
\pi \text {-loop }\end{array}$ & $\begin{array}{c}\frac{g_{1}^{2}}{2 F_{0}^{2}}+\frac{g_{2}^{2}}{F_{0}^{2}}+\frac{5 g_{5}^{2}}{4 F_{0}^{2}} \\
\frac{g_{1}^{2}}{6 F_{0}^{2}}+\frac{g_{5}^{2}}{24 F_{0}^{2}} \\
\frac{g_{1}^{2}}{F_{0}^{2}}+\frac{g_{2}^{2}}{F_{0}^{2}}+\frac{3 g_{5}^{2}}{8 F_{0}^{2}} \\
\end{array}$ & $\begin{array}{l}-\frac{7 g_{3}^{2}}{16 F_{0}^{2}}-\frac{g_{4}^{2}}{8 F_{0}^{2}} \\
-\frac{5 g_{3}^{2}}{96 F_{0}^{2}}-\frac{3 g_{4}^{2}}{16 F_{0}^{2}} \\
-\frac{11 g_{3}^{2}}{32 F_{0}^{2}}-\frac{3 g_{4}^{2}}{16 F_{0}^{2}} \\
\end{array}$ \\
\hline$\lambda_{\Xi_{c}^{* \prime}+\Omega_{c}^{0}}$ & $\begin{array}{l}K \text {-loop } \\
\eta \text {-loop } \\
\pi \text {-loop }\end{array}$ & $\begin{array}{c}\frac{g_{1}^{2}}{F_{0}^{2}}+\frac{2 g_{2}^{2}}{F_{0}^{2}}+\frac{5 g_{5}^{2}}{4 F_{0}^{2}} \\
\frac{2 g_{1}^{2}}{3 F_{0}^{2}}+\frac{g_{5}^{2}}{24 F_{0}^{2}} \\
\frac{3 g_{5}^{2}}{8 F_{0}^{2}}\end{array}$ & $\begin{array}{l}-\frac{9 g_{3}^{2}}{16 F_{0}^{2}}-\frac{g_{4}^{2}}{8 F_{0}^{2}} \\
-\frac{17 g_{3}^{2}}{96 F_{0}^{2}}-\frac{3 g_{4}^{2}}{16 F_{0}^{2}} \\
-\frac{3 g_{3}^{2}}{32 F_{0}^{2}}-\frac{3 g_{4}^{2}}{16 F_{0}^{2}}\end{array}$ \\
\hline$\lambda_{\Sigma_{c}^{*+} \Xi_{c}^{\prime+}}$ & $\begin{array}{l}K \text {-loop } \\
\eta \text {-loop } \\
\pi \text {-loop }\end{array}$ & $\begin{array}{l}\frac{5 g_{1}^{2}}{4 F_{0}^{2}}+\frac{g_{2}^{2}}{2 F_{0}^{2}}+\frac{g_{5}^{2}}{2 F_{0}^{2}} \\
\frac{g_{1}^{2}}{24 F_{0}^{2}}+\frac{3 g_{2}^{2}}{4 F_{0}^{2}}+\frac{g_{5}^{2}}{6 F_{0}^{2}} \\
\frac{3 g_{1}^{2}}{8 F_{0}^{2}}+\frac{3 g_{2}^{2}}{4 F_{0}^{2}}+\frac{g_{5}^{2}}{F_{0}^{2}}\end{array}$ & $\begin{array}{c}-\frac{7 g_{3}^{2}}{16 F_{0}^{2}}-\frac{g_{4}^{2}}{4 F_{0}^{2}} \\
\quad-\frac{5 g_{3}^{2}}{96 F_{0}^{2}} \\
-\frac{11 g_{3}^{2}}{32 F_{0}^{2}}-\frac{g_{4}^{2}}{4 F_{0}^{2}} \\
\end{array}$ \\
\hline$\lambda_{\Sigma_{c}^{*++}} \Xi_{c}^{\prime+}$ & $\begin{array}{l}K \text {-loop } \\
\eta \text {-loop } \\
\pi \text {-loop }\end{array}$ & $\begin{array}{l}\frac{5 g_{1}^{2}}{4 F_{0}^{2}}+\frac{g_{2}^{2}}{2 F_{0}^{2}}+\frac{g_{5}^{2}}{2 F_{0}^{2}} \\
\frac{g_{1}^{2}}{24 F_{0}^{2}}+\frac{3 g_{2}^{2}}{4 F_{0}^{2}}+\frac{g_{5}^{2}}{6 F_{0}^{2}} \\
\frac{3 g_{1}^{2}}{8 F_{0}^{2}}+\frac{3 g_{2}^{2}}{4 F_{0}^{2}}+\frac{g_{5}^{2}}{F_{0}^{2}}\end{array}$ & $\begin{array}{c}-\frac{7 g_{3}^{2}}{16 F_{0}^{2}}-\frac{g_{4}^{2}}{4 F_{0}^{2}} \\
\quad-\frac{5 g_{3}^{2}}{96 F_{0}^{2}} \\
-\frac{11 g_{3}^{2}}{32 F_{0}^{2}}-\frac{g_{4}^{2}}{4 F_{0}^{2}} \\
\end{array}$ \\
\hline
\end{tabular}


TABLE XVIII: The coefficients $\lambda_{4(i j)}$ of the axial current from the wave function renormalization.

\begin{tabular}{|c|c|c|c|}
\hline & & Case I & Case II \\
\hline$\lambda_{\Lambda_{c}^{+} \Sigma_{c}^{* 0}}$ & $\begin{array}{l}K \text {-loop } \\
\eta \text {-loop } \\
\pi \text {-loop }\end{array}$ & $\begin{array}{c}\frac{g_{2}^{2}}{F_{0}^{2}}+\frac{g_{5}^{2}}{2 F_{0}^{2}}+\frac{2 g_{6}^{2}}{F_{0}^{2}} \\
\frac{g_{5}^{2}}{6 F_{0}^{2}}+\frac{2 g_{6}^{2}}{3 F_{0}^{2}} \\
\frac{3 g_{2}^{2}}{F_{0}^{2}}+\frac{g_{5}^{2}}{F_{0}^{2}} \\
\end{array}$ & $\begin{array}{c}-\frac{g_{3}^{2}}{8 F_{0}^{2}}-\frac{g_{4}^{2}}{2 F_{0}^{2}} \\
-\frac{g_{3}^{2}}{24 F_{0}^{2}} \\
-\frac{g_{3}^{2}}{4 F_{0}^{2}}-\frac{g_{4}^{2}}{F_{0}^{2}}\end{array}$ \\
\hline$\lambda_{\Xi_{c}^{+} \Xi_{c}^{* \prime 0}}$ & $\begin{array}{l}K \text {-loop } \\
\eta \text {-loop } \\
\pi \text {-loop }\end{array}$ & $\begin{array}{l}\frac{5 g_{2}^{2}}{2 F_{0}^{2}}+\frac{5 g_{5}^{2}}{4 F_{0}^{2}}+\frac{g_{6}^{2}}{F_{0}^{2}} \\
\frac{3 g_{2}^{2}}{4 F_{0}^{2}}+\frac{g_{5}^{2}}{24 F_{0}^{2}}+\frac{g_{6}^{2}}{6 F_{0}^{2}} \\
\frac{3 g_{2}^{2}}{4 F_{0}^{2}}+\frac{3 g_{5}^{2}}{8 F_{0}^{2}}+\frac{3 g_{6}^{2}}{2 F_{0}^{2}}\end{array}$ & $\begin{array}{l}-\frac{5 g_{3}^{2}}{16 F_{0}^{2}}-\frac{3 g_{4}^{2}}{4 F_{0}^{2}} \\
-\frac{g_{3}^{2}}{96 F_{0}^{2}}-\frac{3 g_{4}^{2}}{8 F_{0}^{2}} \\
-\frac{3 g_{3}^{2}}{32 F_{0}^{2}}-\frac{3 g_{4}^{2}}{8 F_{0}^{2}}\end{array}$ \\
\hline$\lambda_{\Lambda_{c}^{+} \Xi_{c}^{* \prime 0}}$ & $\begin{array}{l}K \text {-loop } \\
\eta \text {-loop } \\
\pi \text {-loop }\end{array}$ & $\begin{array}{c}\frac{g_{2}^{2}}{F_{0}^{2}}+\frac{5 g_{5}^{2}}{4 F_{0}^{2}}+\frac{2 g_{6}^{2}}{F_{0}^{2}} \\
\frac{g_{5}^{2}}{24 F_{0}^{2}}+\frac{2 g_{6}^{2}}{3 F_{0}^{2}} \\
\frac{3 g_{2}^{2}}{F_{0}^{2}}+\frac{3 g_{5}^{2}}{8 F_{0}^{2}} \\
\end{array}$ & $\begin{array}{l}-\frac{5 g_{3}^{2}}{16 F_{0}^{2}}-\frac{3 g_{4}^{2}}{8 F_{0}^{2}} \\
-\frac{g_{3}^{2}}{96 F_{0}^{2}}-\frac{3 g_{4}^{2}}{16 F_{0}^{2}} \\
-\frac{3 g_{3}^{2}}{32 F_{0}^{2}}-\frac{15 g_{4}^{2}}{16 F_{0}^{2}}\end{array}$ \\
\hline$\lambda_{\Xi_{c}^{+} \Omega_{c}^{* 0}}$ & $\begin{array}{l}K \text {-loop } \\
\eta \text {-loop } \\
\pi \text {-loop }\end{array}$ & $\begin{array}{c}\frac{5 g_{2}^{2}}{2 F_{0}^{2}}+\frac{g_{5}^{2}}{F_{0}^{2}}+\frac{g_{6}^{2}}{F_{0}^{2}} \\
\frac{3 g_{2}^{2}}{4 F_{0}^{2}}+\frac{2 g_{5}^{2}}{3 F_{0}^{2}}+\frac{g_{6}^{2}}{6 F_{0}^{2}} \\
\frac{3 g_{2}^{2}}{4 F_{0}^{2}}+\frac{3 g_{6}^{2}}{2 F_{0}^{2}}\end{array}$ & $\begin{array}{c}-\frac{g_{3}^{2}}{4 F_{0}^{2}}-\frac{9 g_{4}^{2}}{8 F_{0}^{2}} \\
-\frac{g_{3}^{2}}{6 F_{0}^{2}}-\frac{3 g_{4}^{2}}{16 F_{0}^{2}} \\
-\frac{3 g_{4}^{2}}{16 F_{0}^{2}}\end{array}$ \\
\hline
\end{tabular}




\section{INTEGRALS AND FUNCTIONS}

1. The integral with one meson line and one baryon line in Fig. 1 ,

$$
\left\{\begin{array}{l}
J(m, \omega)=\frac{1}{8 \pi^{2}}\left[\omega(R-1)+\omega \ln \frac{m^{2}}{\mu^{2}}+K\right], \omega=v \cdot k+\delta \\
I(m)=\frac{m^{2}}{16 \pi^{2}}\left(R+\ln \frac{m^{2}}{\mu^{2}}\right)
\end{array}\right.
$$

and

$$
K=\left\{\begin{array}{c}
2 \sqrt{\omega^{2}-m^{2}} \operatorname{arccosh} \frac{\omega}{m}-2 i \pi \sqrt{\omega^{2}-m^{2}}, \quad \omega>m \\
-2 \sqrt{\omega^{2}-m^{2}} \operatorname{arccosh} \frac{-\omega}{m}, \quad \omega<-m \\
2 \sqrt{m^{2}-\omega^{2}} \arccos \frac{-\omega}{m}, \quad \omega^{2}<m^{2} \\
J_{\alpha \beta}=C_{21} g_{\alpha \beta}+C_{20} v_{\alpha} v_{\beta}
\end{array}\right.
$$

where

$$
C_{21}=\frac{1}{d-1}\left[\left(m^{2}-\omega^{2}\right) J(m, \omega)+\omega I(m)\right]=\frac{1}{d-1} f(m, \omega)
$$

The definition of $f$ can be read from above easily.

2. The integrals with one meson line and two baryon lines in Figs. 37 ;

When the masses of the two baryons in diagram (a) are the same, we introduce the integrals

$$
\left\{L, L_{\mu}, L_{\mu \nu}\right\}=\frac{1}{i} \int d^{d} q \frac{\left\{1, q_{\mu}, q_{\mu} q_{\nu}\right\}}{\left(m^{2}-q^{2}-i \varepsilon\right)[v \cdot q+\omega+i \varepsilon]^{2}}
$$

using

$$
\frac{1}{[v \cdot q+\omega]^{2}}=-\frac{\partial}{\partial \omega} \frac{1}{[v \cdot q+\omega]}
$$

There is a relation between $L$ and $J$

$$
\left\{L, L_{\mu}, L_{\mu \nu}\right\}=-\frac{\partial}{\partial \alpha}\left\{J, J_{\mu}, J_{\mu \nu}\right\}
$$

When the masses of the two baryons in diagram (a) are different, we define the integrals

$$
\left\{F, F_{\mu}, F_{\mu \nu}\right\}=\frac{1}{i} \int d^{d} q \frac{\left\{1, q_{\mu}, q_{\mu} q_{\nu}\right\}}{\left(m^{2}-q^{2}-i \varepsilon\right)\left[v \cdot q+\omega_{1}+i \varepsilon\right]\left[v \cdot q+\omega_{2}+i \varepsilon\right]}
$$

using

$$
\frac{1}{\left[v \cdot q+\omega_{1}\right]\left[v \cdot q+\omega_{2}\right]}=-\frac{1}{\omega_{1}-\omega_{2}}\left(\frac{1}{\left[v \cdot q+\omega_{1}\right]}-\frac{1}{\left[v \cdot q+\omega_{2}\right]}\right)
$$

The relation between $F$ and $J$ is

$$
\left\{F, F_{\mu}, F_{\mu \nu}\right\}=-\frac{1}{\omega_{1}-\omega_{2}}\left\{J\left(\omega_{1}\right)-J\left(\omega_{2}\right), J_{\mu}\left(\omega_{1}\right)-J_{\mu}\left(\omega_{2}\right), J_{\mu \nu}\left(\omega_{1}\right)-J_{\mu \nu}\left(\omega_{2}\right)\right\}
$$

Especially, for the second-order tensor formula, $F_{\alpha \beta}$ and $L_{\alpha \beta}$ can be expressed as a sum of the two Lorentz structures. $F_{\alpha \beta}^{20}$ and $L_{\alpha \beta}^{20}$ are proportional to $v^{\alpha} v^{\beta}$ and vanish when contracted with $S^{\mu}$ and $\mathcal{T}^{\mu}$. So, we are concerned about the remaining part only:

$$
F_{\alpha \beta}=-\frac{1}{\omega_{1}-\omega_{2}}\left(J_{\alpha \beta}\left(\omega_{1}\right)-J_{\alpha \beta}\left(\omega_{2}\right)\right)=F_{\alpha \beta}^{21}+F_{\alpha \beta}^{20}
$$




$$
\begin{gathered}
F_{\alpha \beta}^{21}=g_{\alpha \beta} \frac{-1}{d-1} \frac{f\left(m, \omega_{1}\right)-f\left(m, \omega_{2}\right)}{\omega_{1}-\omega_{2}} \\
L_{\alpha \beta}=-\frac{\partial}{\partial \omega} J_{\alpha \beta}(\omega)=L_{\alpha \beta}^{21}+L_{\alpha \beta}^{20} \\
L_{\alpha \beta}^{21}=g_{\alpha \beta} \frac{-1}{d-1} \frac{\partial f(m, \omega)}{\partial \omega}
\end{gathered}
$$

$F_{\alpha \beta}^{21}$ and $L_{\alpha \beta}^{21}$ can be uniformed as

$$
g_{\alpha \beta} \frac{-1}{d-1} \frac{\Delta f}{\Delta \omega}
$$

where

$$
\frac{\Delta f}{\Delta \omega}=\left\{\begin{array}{lr}
m^{2} \frac{\Delta J(\omega)}{\Delta \omega}-\frac{\Delta\left(\omega^{2} J(\omega)\right)}{\Delta \omega}+I(m), & \omega_{1} \neq \omega_{2} \\
\left(m^{2}-\omega^{2}\right) \frac{\partial J(\omega)}{\partial \omega}-2 \omega J(\omega)+I(m), & \omega_{1}=\omega_{2}=\omega
\end{array}\right.
$$

and $\frac{\Delta J(\omega)}{\Delta \omega}$ denotes $\frac{J\left(\omega_{1}\right)-J\left(\omega_{2}\right)}{\omega_{1}-\omega_{2}}$, and similar conventions hold for $\frac{\Delta\left(\omega^{2} J(\omega)\right)}{\Delta \omega}$ and $\frac{\Delta\left(\omega^{3}\right)}{\Delta \omega}$. Combining with the parameters $a$ and $b$, the integral from diagram (a) can be written as

$$
(a+b \epsilon) \frac{\Delta f}{\Delta \omega}=a\left\{\begin{array}{cc}
m^{2} \frac{\Delta J}{\Delta \omega}-\frac{\Delta\left(\omega^{2} J\right)}{\Delta \omega}+I, & \left(\omega_{1} \neq \omega_{2}\right) \\
\left(m^{2}-\omega^{2}\right) \frac{\partial J}{\partial \omega}-2 \omega J(\omega)+I, & \left(\omega_{1}=\omega_{2}=\omega\right)
\end{array}\right\}-\frac{b}{4 \pi^{2}}\left[\frac{3}{2} m^{2}-\frac{\Delta\left(\omega^{3}\right)}{\Delta \omega}\right]
$$

\section{THE CONTRACTION FORMULAS FOR $S^{\mu}$ and $P_{(33) \mu \nu}^{\frac{3}{2}}$}

The Pauli-Lubanski vector $S^{\mu}$ and projection operator $P_{(33) \mu \nu}^{\frac{3}{2}}$ are defined as follows:

$$
\begin{aligned}
S^{\mu} & =-\frac{1}{2} \gamma_{5}\left(\gamma^{\mu} \psi-v^{\mu}\right) \\
P_{(33) \mu \nu}^{\frac{3}{2}} & =g_{\mu \nu}-v_{\mu} v_{\nu}+\frac{4}{d-1} S_{\mu} S_{\nu}
\end{aligned}
$$

In the calculation of the loop correction of the self-energy function and axial charges, the following formulas are very useful.

$$
\begin{aligned}
P^{\rho \sigma} g_{\rho \sigma} & =d-2 \\
S^{\sigma} P^{\rho \mu} & =\frac{4}{d-1} S^{\rho} S^{\mu} S^{\sigma}+g_{\perp}^{\rho \mu} S^{\sigma}+\frac{2}{d-1} S^{\rho} g_{\perp}^{\mu \sigma}-\frac{2}{d-1} g_{\perp}^{\rho \sigma} S^{\mu} \\
P^{\mu \sigma} S^{\rho}= & \frac{4}{d-1} S^{\rho} S^{\mu} S^{\sigma}+S^{\rho} g_{\perp}^{\mu \sigma}+\frac{2}{d-1} g_{\perp}^{\rho \mu} S^{\sigma}-\frac{2}{d-1} g_{\perp}^{\rho \sigma} S^{\mu} \\
S_{\alpha} P^{\mu \alpha}= & \frac{2(d-2)}{d-1} S^{\mu} \\
S^{\alpha} P^{\rho \sigma} S_{\alpha}= & \left(\frac{1-d}{4}+\frac{1}{d-1}\right) g_{\perp}^{\rho \sigma}+\frac{5-d}{d-1} S^{\rho} S^{\sigma} \\
P^{\rho \lambda} S^{\mu} P_{\lambda}^{\sigma}= & -\frac{4(d+1)}{(d-1)^{2}} S^{\rho} S^{\mu} S^{\sigma}+S^{\mu} g_{\perp}^{\rho \sigma}-\frac{2}{d-1}\left(g_{\perp}^{\rho \mu} S^{\sigma}+S^{\rho} g_{\perp}^{\mu \sigma}\right) \\
P^{\rho \lambda} S^{\mu} P_{\lambda \rho}= & \frac{(d-3)(d-2)(d+1)}{(d-1)^{2}} S^{\mu}
\end{aligned}
$$




$$
\begin{aligned}
P^{\alpha \lambda} S^{\mu} P_{\lambda}{ }^{\sigma} S_{\alpha}= & \frac{2(d-3)(d+1)}{(d-1)^{2}} S^{\mu} S^{\sigma}+\frac{(d-3)(d+1)}{2(d-1)^{2}} g_{\perp}^{\mu \sigma} \\
S^{\alpha} P^{\rho \lambda} S^{\mu} P_{\lambda}{ }^{\sigma} S_{\alpha}= & -\frac{(d+1)(d-7)}{(d-1)^{2}} S^{\rho} S^{\mu} S^{\sigma}-\frac{d^{2}-6 d+1}{2(d-1)^{2}}\left(S^{\rho} g_{\perp}^{\mu \sigma}+g_{\perp}^{\rho \mu} S^{\sigma}\right) \\
& +\frac{d^{3}-5 d^{2}+3 d-7}{4(d-1)^{2}} S^{\mu} g_{\perp}^{\rho \sigma}
\end{aligned}
$$


[1] J. J. Aubert et al. [E598 Collaboration], Phys. Rev. Lett. 33, 1404 (1974).

[2] J. E. Augustin et al. [SLAC-SP-017 Collaboration], Phys. Rev. Lett. 33 (1974) 1406.

[3] S. W. Herb, D. C. Hom, L. M. Lederman, J. C. Sens, H. D. Snyder, J. K. Yoh, J. A. Appel and B. C. Brown et al., Phys. Rev. Lett. 39, 252 (1977).

[4] W. R. Innes, J. A. Appel, B. C. Brown, C. N. Brown, K. Ueno, T. Yamanouchi, S. W. Herb and D. C. Hom et al., Phys. Rev. Lett. 39, 1240 (1977) [Erratum-ibid. 39, 1640 (1977)].

[5] K. Nakamura et al. [Particle Data Group Collaboration], J. Phys. G 37, 075021 (2010).

[6] F. Wick [CDF Collaboration], Int. J. Mod. Phys. Conf. Ser. 02, 163 (2011) arXiv:1105.0517 [hep-ex]].

[7] T. Aaltonen et al. [CDF Collaboration], Phys. Rev. D 84, 012003 (2011) arXiv:1105.5995 [hep-ex]].

[8] Z. -W. Liu and S. -L. Zhu, Phys. Rev. D 86, 034009 (2012) arXiv:1205.0467 [hep-ph]].

[9] Z. -W. Liu and S. -L. Zhu, Nucl. Phys. A 914, 494 (2013) arXiv:1211.5013 [hep-ph]].

[10] N. Li and S. -L. Zhu, Phys. Rev. D 86, 014020 (2012) [arXiv:1204.3364 [hep-ph]].

[11] E. E. Jenkins and A. V. Manohar, Phys. Lett. B 255, 558 (1991).

[12] E. E. Jenkins and A. V. Manohar, Phys. Lett. B 259, 353 (1991).

[13] S. -L. Zhu, S. Puglia and M. J. Ramsey-Musolf, Phys. Rev. D 63, 034002 (2001) hep-ph/0009159.

[14] S. -L. Zhu, G. Sacco and M. J. Ramsey-Musolf, Phys. Rev. D 66, 034021 (2002) hep-ph/0201179.

[15] S. Weinberg, Physica A 96, 327 (1979).

[16] J. Gasser and H. Leutwyler, Annals Phys. 158, 142 (1984).

[17] J. Gasser and H. Leutwyler, Nucl. Phys. B 250, 465 (1985).

[18] J. Gasser, M. E. Sainio and A. Svarc, Nucl. Phys. B 307, 779 (1988).

[19] V. Bernard, N. Kaiser, J. Kambor and U. G. Meissner, Nucl. Phys. B 388, 315 (1992).

[20] V. Bernard, N. Kaiser and U. G. Meissner, Nucl. Phys. B 383, 442 (1992).

[21] S. Scherer, Adv. Nucl. Phys. 27, 277 (2003) hep-ph/0210398.

[22] V. Bernard, N. Kaiser and U. -G. Meissner, Int. J. Mod. Phys. E 4, 193 (1995) hep-ph/9501384.

[23] V. Bernard, N. Kaiser and U. -G. Meissner, Nucl. Phys. A 615, 483 (1997) hep-ph/9611253.

[24] M. Mojzis, Eur. Phys. J. C 2, 181 (1998) hep-ph/9704415.

[25] N. Fettes, U. -G. Meissner and S. Steininger, Nucl. Phys. A 640, 199 (1998) hep-ph/9803266.

[26] N. Fettes and U. -G. Meissner, Nucl. Phys. A 693, 693 (2001) hep-ph/0101030.

[27] T. Becher and H. Leutwyler, Eur. Phys. J. C 9, 643 (1999) hep-ph/9901384.

[28] K. Johnson and E. C. G. Sudarshan, Annals Phys. 13, 126 (1961).

[29] T. -M. Yan, H. -Y. Cheng, C. -Y. Cheung, G. -L. Lin, Y. C. Lin and H. -L. Yu, Phys. Rev. D 46, 1148 (1992) [Erratum-ibid. D 55, 5851 (1997)].

[30] N. Fettes, U. -G. Meissner, M. Mojzis and S. Steininger, Annals Phys. 283, 273 (2000) [Erratum-ibid. 288, 249 (2001)] hep-ph/0001308.

[31] M. Benmerrouche, R. M. Davidson and N. C. Mukhopadhyay, Phys. Rev. C 39, 2339 (1989).

[32] T. R. Hemmert, B. R. Holstein and J. Kambor, J. Phys. G 24, 1831 (1998) hep-ph/9712496.

[33] T. Pilling, Int. J. Mod. Phys. A 20, 2715 (2005) hep-th/0404131.

[34] F. -K. Guo, C. Hanhart and U. -G. Meissner, JHEP 0809, 136 (2008) arXiv:0809.2359 [hep-ph]].

[35] A. V. Manohar and M. B. Wise, Camb. Monogr. Part. Phys. Nucl. Phys. Cosmol. 10, 1 (2000).

[36] N. Mathur, R. Lewis and R. M. Woloshyn, Phys. Rev. D 66, 014502 (2002) hep-ph/0203253.

[37] W. Detmold, C. -J. D. Lin and S. Meinel, Phys. Rev. D 84, 094502 (2011) arXiv:1108.5594 [hep-lat]]. 